\title{
Improved Non-Local Means Algorithm Based on Dimensionality Reduction
}

Golam Morshed Maruf, The University of Western Ontario

Supervisor: Dr. Mahmoud R. El-Sakka, The University of Western Ontario

A thesis submitted in partial fulfillment of the requirements for the Master of Science degree in Computer Science

(C) Golam Morshed Maruf 2014

Follow this and additional works at: https://ir.lib.uwo.ca/etd

\section{Recommended Citation}

Maruf, Golam Morshed, "Improved Non-Local Means Algorithm Based on Dimensionality Reduction" (2014). Electronic Thesis and Dissertation Repository. 2615.

https://ir.lib.uwo.ca/etd/2615

This Dissertation/Thesis is brought to you for free and open access by Scholarship@Western. It has been accepted for inclusion in Electronic Thesis and Dissertation Repository by an authorized administrator of Scholarship@Western. For more information, please contact wlswadmin@uwo.ca. 


\section{IMPROVED NON-LOCAL MEANS ALGORITHM BASED ON DIMENSIONALITY REDUCTION}

(Thesis format: Monograph)

by

Golam Morshed Maruf

Graduate Program in Department of Computer Science

A thesis submitted in partial fulfillment of the requirements for the degree of Masters of Science

The School of Graduate and Postdoctoral Studies

The University of Western Ontario

London, Ontario, Canada

(C) Golam Morshed Maruf 2014 


\begin{abstract}
Non-Local Means is an image denoising algorithm based on patch similarity. It compares a reference patch with the neighboring patches to find similar patches. Such similar patches participate in the weighted averaging process. Most of the computational time for Non-Local Means is consumed to measure patch similarity. In this thesis, we have proposed an improvement where the image patches are projected into a global feature space. Then we have performed a statistical t-test to reduce the dimensionality of this feature space. Denoising is achieved based on this reduced feature space and the proposed modification exploits an improvement in terms of denoising performance and computational time.
\end{abstract}

\title{
Keywords
}

Non-Local Means algorithm, image denoising, image smoothing, image enhancement, additive white gaussian noise, spatial domain filtering. 


\section{Dedication}

To

My loving parents and sister

M.A. Malek, Mahera Begum and Nusrat Mahin

without whom my success would be impossible. 


\section{Acknowledgments}

This dissertation has been accomplished under the supervision of Dr. Mahmoud R. El-Sakka in the Department of Computer Science, University of Western Ontario.

I am expressing my heartiest gratitude to the Almighty, most gracious and most merciful to give me the ability to complete my thesis successfully. I would like to thank my thesis supervisor Dr. Mahmoud R. El-Sakka for his noteworthy and valuable direction, guidance, motivation and encouragement in the way of my progress. Moreover, I am also thankful to all of my professors from the University of Western Ontario for building my background to complete this task.

Lastly, I am grateful to my parents for their continuous support and guidance. 


\section{Table of Contents}

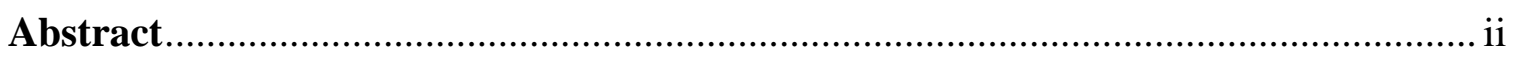

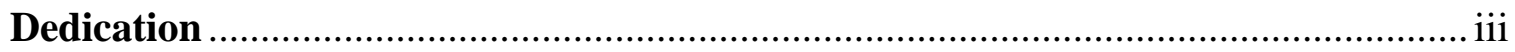

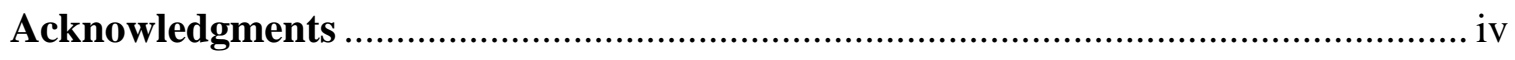

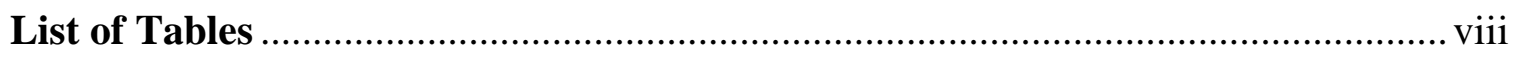

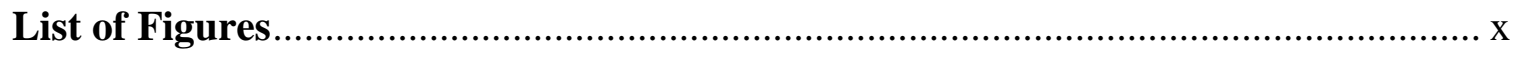

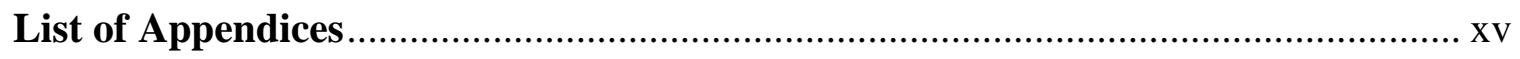

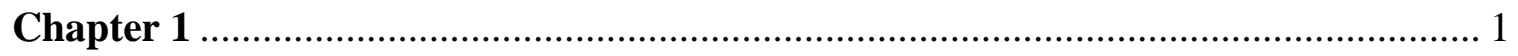

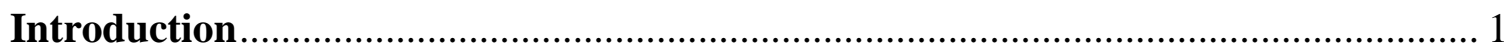

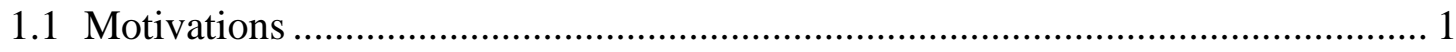

1.2 Thesis contributions ................................................................................. 2

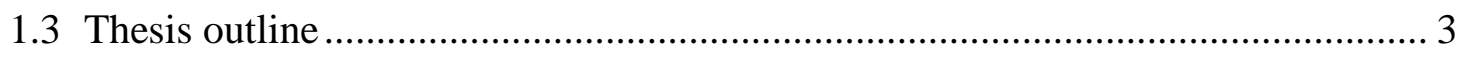

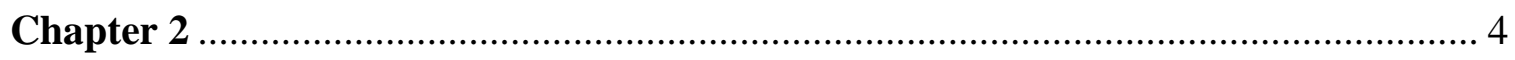

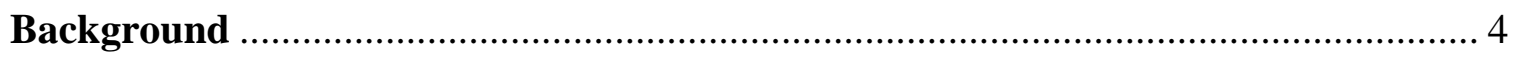

2.1 Additive white Gaussian noise................................................................... 4

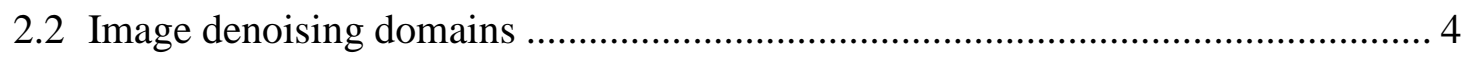

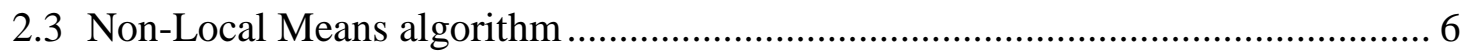

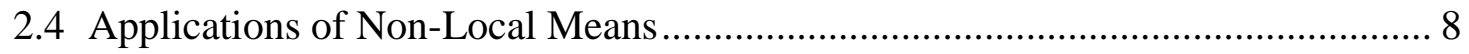

2.5 Improvement over Non-Local Means ................................................... 9

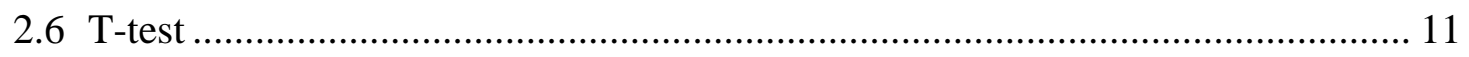

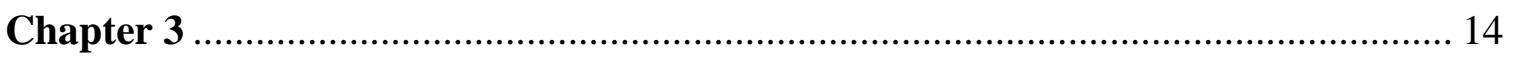

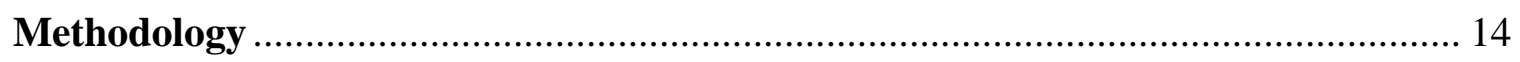

3.1 Improved Non-Local Means Algorithm ...................................................... 14 


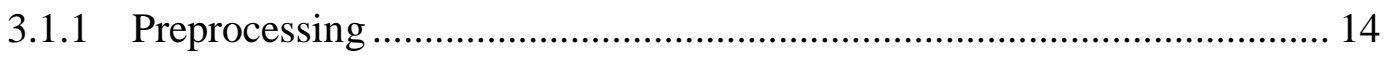

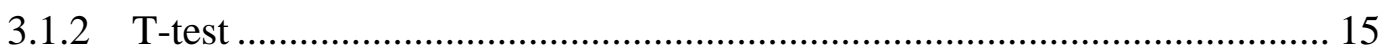

3.1.3 Non-Local Means algorithm ............................................................... 16

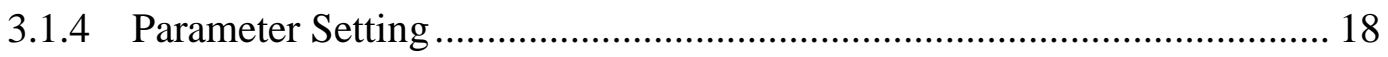

3.1.5 Selected Parameters .......................................................................... 31

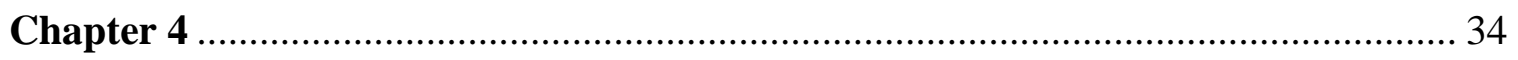

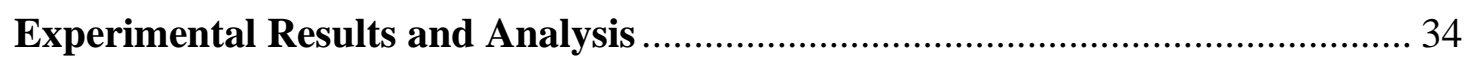

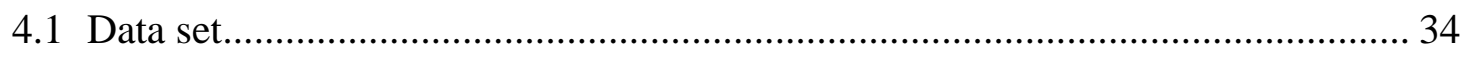

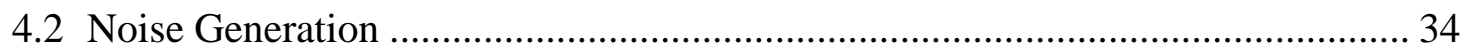

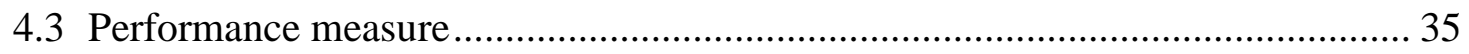

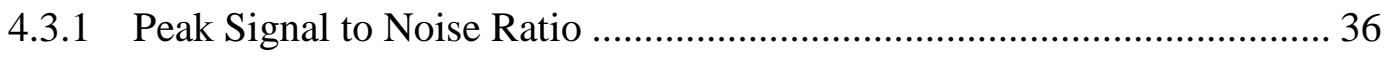

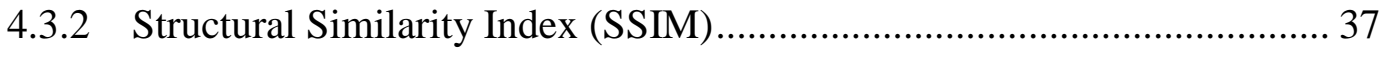

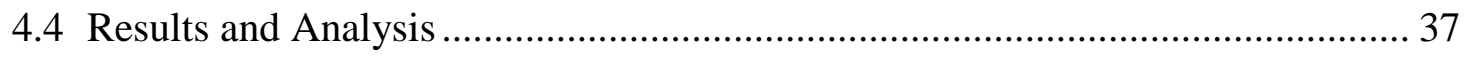

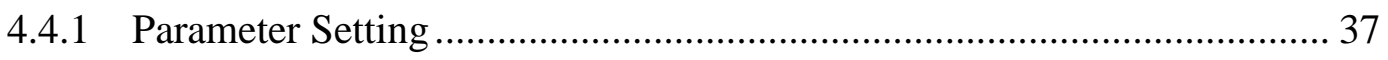

4.4.2 Performance analysis using PSNR ......................................................... 38

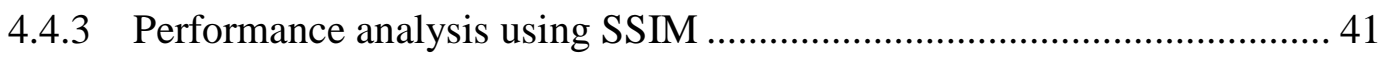

4.4.4 Running time performance analysis........................................................ 44

4.4.5 Visual quality comparison and intensity profile ……………………….... 47

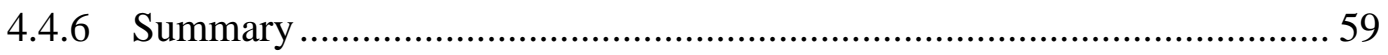

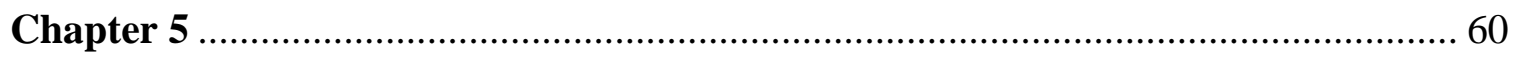

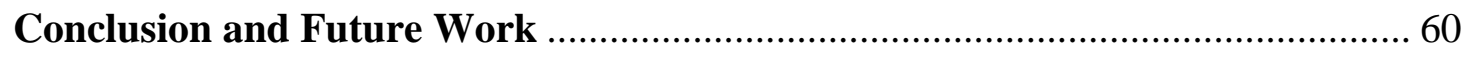

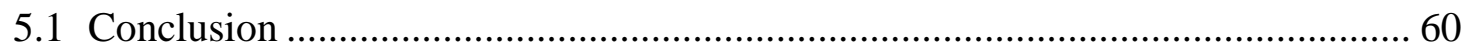

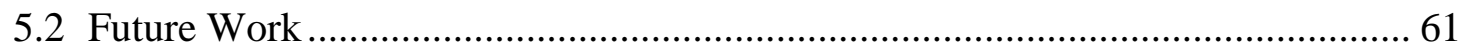

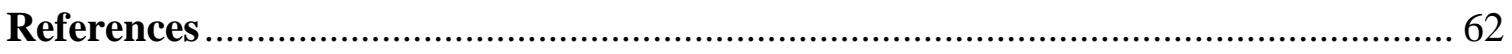




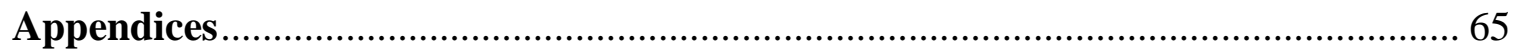

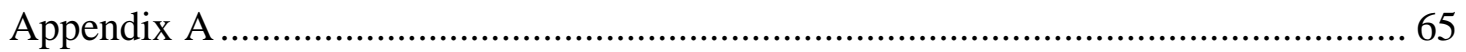

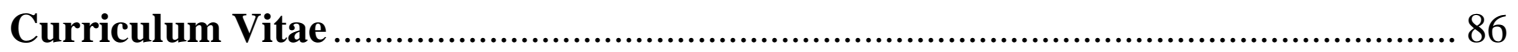




\section{List of Tables}

Table 3- 1: Average PSNR comparison for all test images between different patch sizes for different noise levels.

Table 3- 2: Average PSNR comparison for all test images between different search region sizes for different noise levels. 23

Table 3- 3: Average PSNR and average number of features comparison for patch size $7 \times 7$ for all test images between different threshold values for different noise levels. 26

Table 3- 4: Average PSNR and average number of features comparison for patch size $5 \times 5$ for all test images between different threshold values for different noise levels. 27

Table 3- 5: Average PSNR comparison for all test images between different patch sizes for different noise levels. 32

Table 3- 6: Average PSNR comparison for all test images between different patch sizes for different noise levels. 33

Table 4-1: PSNR(dB) comparison for Lena image among the proposed method, the NLM method, variants of the NLM method and the BM3D method for different noise levels...... 39

Table 4-2: Average PSNR(dB) comparison for all test images among the proposed method, the NLM method, variants of the NLM method and the BM3D method for different noise levels. 40

Table 4-3: SSIM comparison for Lena image among the proposed method, the NLM method, variants of the NLM method and the BM3D method for different noise levels. 42

Table 4-4: Average SSIM comparison for all test images among the proposed method, the NLM method, variants of the NLM method and the BM3D method for different noise levels. 
Table 4-5: Running time (in milliseconds) performance analysis for Lena image among the proposed method, the NLM method, variants of the NLM method and the BM3D method for different noise levels. 45

Table 4-6: Average running time (in milliseconds) performance analysis for all test images among the proposed method, the NLM method, variants of the NLM method and the BM3D method for different noise levels. 46

Table A- 1: PSNR (dB) comparison for standard Peepers image between our proposed method and other denoising algorithms .65

Table A- 2: SSIM comparison for image Peppers between proposed method and other denoising algorithms for different noise level. 66

Table A- 3: PSNR (dB) comparison for standard Boat image between our proposed method and other denoising algorithms. 72

Table A- 4: SSIM comparison for image Boat between proposed method and other denoising algorithms for different noise level.

Table A- 5: PSNR (dB) comparison for standard Couple image between our proposed method and other denoising algorithms. 79

Table A- 6: SSIM comparison for image Couple between proposed method and other denoising algorithms for different noise level. 


\section{List of Figures}

Figure 2-1: Probability Distribution for Gaussian noise. 5

Figure 2-2:The Non-Local Means scheme where similar patches $q 1$ and $q 2$ are assigned

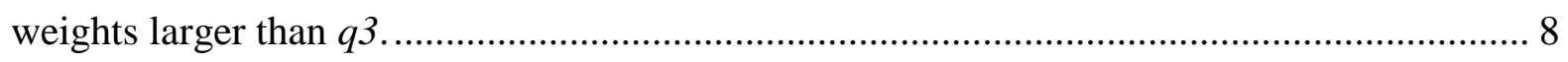

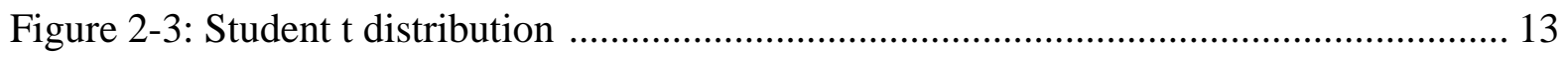

Figure 3 - 1: Set of test images $(512 \times 512)$ for performance analysis $\ldots \ldots \ldots \ldots \ldots \ldots \ldots \ldots$

Figure 3 - 2: Performance analysis using Woman 1 image over different patch sizes where noise level is $\sigma=40$. (a) patch size $5 \times 5$, PSNR $=29.55$, (b) patch size $7 \times 7$, PSNR $=29.79$, (c) patch size $9 \times 9$, PSNR $=29.31$, and $(d)$ patch size $11 \times 11$, PSNR $=28.10$. 21

Figure 3 - 3: Performance analysis using Woman 1 image over different search region sizes where noise level is $\sigma=40$ (a) search region size $=14 \times 14$, PSNR $=29.11$, (b) search region size $=21 \times 21$, PSNR $=29.78$, (c) search region size $=28 \times 28$, PSNR $=29.78$, and (d) search

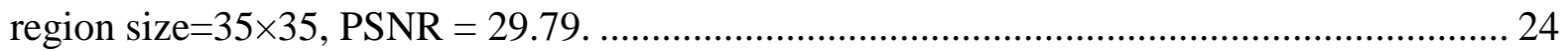

Figure 3 - 4: Average PSNR and average number of features comparison for patch size $7 \times 7$ and threshold value 3 over different noise levels........................................................... 28

Figure 3 - 5: Average PSNR and average number of features comparison for patch size $7 \times 7$ and threshold value 5 over different noise levels.......................................................... 28

Figure 3 - 6: Average PSNR and average number of features comparison for patch size $5 \times 5$ and threshold value 3 over different noise levels. 29

Figure 3 - 7: Average PSNR and average number of features comparison for patch size $5 \times 5$ and threshold value 5 over different noise levels.

Figure 3 - 8: Performance analysis using Woman 1 image over different threshold values where noise level is $\sigma=40$ and patch size is $7 \times 7$ (a) threshold value 3 , PSNR $=29.79$, (b) threshold value 5, PSNR $=29.79$, (c) threshold value 7, PSNR $=29.33$, and (d) threshold value 9, PSNR $=28.60$. 
Figure 4- 1: Set of images for performance analysis.

Figure 4- 2: Noise generation (a) Noise free image Lena. (b) Noisy image with Additive white Gaussian noise (noise level $\sigma=50$ ). 36

Figure 4- 3: Bar graph for average PSNR comparison for the proposed method, the NLM method, variants of the NLM method and the BM3D method for different noise levels 41

Figure 4- 4: Bar graph for average SSIM comparison for all test images among the proposed method, the NLM method, variants of the NLM method and the BM3D method for different noise levels 44

Figure 4- 5: Performance analysis for the average running time (in milliseconds) among the proposed method, the NLM method, variants of the NLM method and the BM3D method for different noise levels. 47

Figure 4- 6: Subjective comparison for denoising performance for Non-Local Means at noise level $\sigma=20$. (a) Noise free image Lena, (b) noisy image with Additive white Gaussian noise, (c) denoised image using the NLM method, PSNR=31.89, and (d) denoised image using the proposed method, PSNR $=33.32$. 49

Figure 4- 7: Subjective comparison for denoising performance for Non-Local Means at noise level $\sigma=40$. (a) Noise free image Lena, (b) noisy image with Additive white Gaussian noise, (c) denoised image using the NLM method, PSNR= 28.42, and (d) denoised image using the proposed method, PSNR $=29.97$ 50

Figure 4- 8: Subjective comparison for denoising performance for Non-Local Means at noise level $\sigma=60$. (a) Noise free image Lena, (b) noisy image with Additive white Gaussian noise, (c) denoised image using the NLM method, PSNR= 25.55, and (d) denoised image using the proposed method, PSNR $=25.98$. 51

Figure 4- 9: Subjective comparison for denoising performance for Non-Local Means at noise level $\sigma=80$. (a) Noise free image Lena, (b) noisy image with Additive white Gaussian noise, (c) denoised image using the NLM method, PSNR= 23.05, and (d) denoised image using the proposed method, PSNR $=23.96$. 
Figure 4- 10: Subjective comparison for denoising performance for Non-Local Means at noise level $\sigma=100$. (a) Noise free image Lena, (b) noisy image with Additive white Gaussian noise, (c) denoised image using the NLM method, PSNR=22.18, and (d) denoised image using the proposed method, PSNR $=22.29$. 53

Figure 4- 11: Output analysis for edge and contrast preservation for Lena image. (a) Original Lena image (b) Noise free fragment of Lena image (c) noisy fragment with $\sigma=40$ (d)denoised fragment using the original NLM method; (d) denoised fragment using the proposed method. 54

Figure 4- 12: Row number 50 of the House image is chosen as the scan line (dark red horizontal line) to generate intensity profiles. 55

Figure 4- 13: Intensity profile of the House image at scan Line $50(\sigma=10)$. 56

Figure 4- 14: Intensity profile of the House image at scan Line $50(\sigma=50)$. 56

Figure 4- 15: Intensity profile of the House image and denoised image by the NLM method at scan Line $50(\sigma=10)$. 57

Figure 4- 16: Intensity profile of the House image and denoised image by the proposed method at scan Line $50(\sigma=10)$. 57

Figure 4- 17: Intensity profile of the House image and denoised image by the NLM at scan Line $50(\sigma=50)$. 58

Figure 4- 18: Intensity profile of House image and denoised image by the proposed method at scan Line $50(\sigma=50)$. 58

Figure A- 1: Subjective comparison for denoising performance for Non-Local Means at noise level $\sigma=20$. (a) Noise free image Peppers. (b) Noisy image with Additive white Gaussian noise. (c) Denoised image with NLM, PSNR= 31.2973. (d) Denoised image with Proposed method, PSNR $=31.7027$. 
Figure A- 2: Subjective comparison for denoising performance for Non-Local Means at noise level $\sigma=40$. (a) Noise free image Peppers. (b) Noisy image with Additive white Gaussian noise. (c) Denoised image with NLM, PSNR= 28.2603. (d) Denoised image with Proposed method, PSNR $=29.7362$. 68

Figure A- 3: Subjective comparison for denoising performance for Non-Local Means at noise level $\sigma=60$. (a) Noise free image Peppers. (b) Noisy image with Additive white Gaussian noise. (c) Denoised image with NLM, PSNR= 24.2415. (d) Denoised image with Proposed method, PSNR = 25.7656.

Figure A- 4: Subjective comparison for denoising performance for Non-Local Means at noise level $\sigma=80$. (a) Noise free image Peppers. (b) Noisy image with Additive white Gaussian noise. (c) Denoised image with NLM, PSNR=23.3125. (d) Denoised image with Proposed method, PSNR $=23.5756$. 70

Figure A- 5: Subjective comparison for denoising performance for Non-Local Means at noise level $\sigma=100$. (a) Noise free image Peppers. (b) Noisy image with Additive white Gaussian noise. (c) Denoised image with NLM, PSNR=21.4862. (d) Denoised image with Proposed method, PSNR $=21.7658$. 71

Figure A- 6: Subjective comparison for denoising performance for Non-Local Means at noise level $\sigma=20$. (a) Noise free image Boat. (b) Noisy image with Additive white Gaussian noise. (c) Denoised image with NLM, PSNR= 29.6207. (d) Denoised image with Proposed method, PSNR $=30.7237$. 74

Figure A- 7: Subjective comparison for denoising performance for Non-Local Means at noise level $\sigma=40$. (a) Noise free image Boat. (b) Noisy image with Additive white Gaussian noise. (c) Denoised image with NLM, PSNR= 26.3797. (d) Denoised image with Proposed method, PSNR $=27.4276$. .75

Figure A- 8: Subjective comparison for denoising performance for Non-Local Means at noise level $\sigma=60$. (a) Noise free image Boat. (b) Noisy image with Additive white Gaussian noise. (c) Denoised image with NLM, PSNR= 23.8791. (d) Denoised image with Proposed method, PSNR $=24.7791$. 76 
Figure A- 9: Subjective comparison for denoising performance for Non-Local Means at noise level $\sigma=80$. (a) Noise free image Boat. (b) Noisy image with Additive white Gaussian noise. (c) Denoised image with NLM, PSNR=22.0718. (d) Denoised image with Proposed method, PSNR $=22.1941$. 77

Figure A- 10: Subjective comparison for denoising performance for Non-Local Means at noise level $\sigma=100$. (a) Noise free image Boat. (b) Noisy image with Additive white Gaussian noise. (c) Denoised image with NLM, PSNR= 20.9146. (d) Denoised image with Proposed method, PSNR $=20.9538$. 78

Figure A- 11: Subjective comparison for denoising performance for Non-Local Means at noise level $\sigma=20$. (a) Noise free image Couple. (b) Noisy image with Additive white Gaussian noise. (c) Denoised image with NLM, PSNR= 30.1471. (d) Denoised image with Proposed method, PSNR $=30.5116$. 81

Figure A- 12: Subjective comparison for denoising performance for Non-Local Means at noise level $\sigma=40$. (a) Noise free image Couple. (b) Noisy image with Additive white Gaussian noise. (c) Denoised image with NLM, PSNR= 26.1758. (d) Denoised image with Proposed method, PSNR $=26.7854$. 82

Figure A- 13: Subjective comparison for denoising performance for Non-Local Means at noise level $\sigma=60$. (a) Noise free image Couple. (b) Noisy image with Additive white Gaussian noise. (c) Denoised image with NLM, PSNR= 23.1847. (d) Denoised image with Proposed method, PSNR $=23.3052$. 83

Figure A- 14: Subjective comparison for denoising performance for Non-Local Means at noise level $\sigma=80$. (a) Noise free image Couple. (b) Noisy image with Additive white Gaussian noise. (c) Denoised image with NLM, PSNR= 21.8258. (d) Denoised image with Proposed method, PSNR $=21.9186$. 84

Figure A- 15: Subjective comparison for denoising performance for Non-Local Means at noise level $\sigma=100$. (a) Noise free image Couple. (b) Noisy image with Additive white Gaussian noise. (c) Denoised image with NLM, PSNR= 20.9146. (d) Denoised image with Proposed method, PSNR $=20.9538$. 85 


\section{List of Appendices}

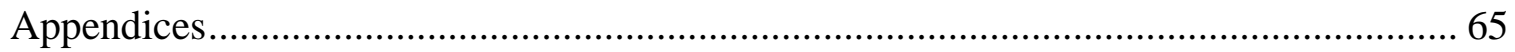

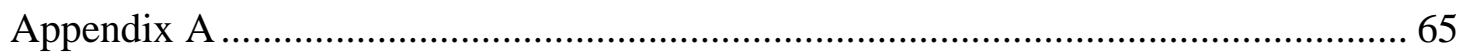




\section{Chapter 1}

\section{Introduction}

An image may be numerically represented as a two dimensional function $u$, in the spatial coordinates $\mathrm{x}$ and $\mathrm{y}$. Intensity or gray level is the amplitude of $u$ at any pair of coordinates. A digital image is composed of finite number of elements called pixels. An image may be contaminated with noise during acquisition, transmission or transformation. Noise is a variation of pixel intensity. Such noise can be additive or multiplicative. Additive noise is generally independent of image data whereas multiplicative noise is dependent on image data. Additive noise can be formularized as,

$$
v(i)=u(i)+n(i)
$$

whereas, multiplicative noise is formularized as,

$$
v(i)=u(i) \times n(i) .
$$

Here, $u(i)$ is the "original" value, $n(i)$ is the "noise" value and $v(i)$ is the "observed" value at pixel $i$. Reducing noise is of great benefit for many applications such as face recognition, object tracking, medical imaging, segmentation. That is why the need of proper image denoising algorithm has grown with much interest. Despite the good quality of acquisition devices, an image denoising method is always required to reduce unwanted signals. Image denoising is used to find the best estimate of the original image from its noisy version. Many methods for image denoising have been proposed in recent years, (see chapter 2).

\subsection{Motivations}

When applying noise reduction algorithms we need to consider several factors, including computational time. Digital cameras need to apply noise reduction at real time using their internal CPU and memory while using computers for denoising can relatively have more processing time. 
Some of the basic filtering such as Gaussian and average filtering have a drawback of over-smoothing on edges and losing image details. Wavelet based denoising method [1], anisotropic diffusion [2], bilateral filtering [3] try to overcome this drawback and preserve the image quality by preserving edges. But they may introduce a staircase effect (makes the image appears like a cartoon image) or false edges. Recently, Buades et al. [4] proposed a denoising algorithm called Non-Local Means (NLM) which allows neighboring patches in the search window to participate in the denoising process for a certain reference patch in the noisy image. Most of the computational time for NLM is allocated to the similarity measure. In a general case, NLM needs to search the entire image for similar patches and performs weighted average based on this similarity. However, searching in a fixed area around the pixel of interest (POI) can reduce this computational time. Our main focus is to further reduce this computational time and improve denoising performance over the original Non-Local Means algorithm.

\subsection{Thesis contributions}

The Non-Local Means algorithm searches neighboring patches to match with the reference patch. The original algorithm requires an extensive amount of time to select patches similar to the reference patch. These similar patches contribute to the weighted averaging process to denoise the center pixel of the reference patch. The computation time for NLM algorithm can be reduced by improving this searching process. In our method, we have created feature vectors for the noisy image. Then we have implemented a statistical t-test on these feature vectors and reduced their dimensionality. These reduced feature vectors contribute to the denoising process. Our proposed method reduces the computational time and improves the overall performance of the original NLM algorithm. 


\subsection{Thesis outline}

We have formalized our thesis into five chapters including this introductory discussion as Chapter 1. In Chapter 2, we discuss Gaussian noise, image denoising domains, the NonLocal Means algorithm, as well as its variants. In addition we present a statistical $t$-test. In Chapter 3, we introduce our proposed method in details and explained its parameters. In Chapter 4, we present our experimental results and compare our proposed method with other denoising algorithms. Finally in Chapter 5, we give our concluding remarks and future work. 


\section{Chapter 2}

\section{Background}

Noise may distort an image and degrade its visual quality. Image denoising schemes attempt to reduce this noise and improve image visual quality. There are many denoising algorithms aiming to reduce noise from digital images. One of the most successful image denoising scheme is the Non-Local Means (NLM) algorithm. In this chapter, the NonLocal Means algorithm and its improvements as well as statistical $t$-test will be discussed.

\subsection{Additive white Gaussian noise}

White noise is a random signal with a constant power spectral density. Gaussian noise is a statistical noise having normal distribution. The probability density function (PDF) of a white Gaussian noise is given by,

$$
P D F(z)=\frac{1}{\sigma \sqrt{2 \pi}} e^{-\frac{(z-\mu)^{2}}{2 \sigma^{2}}}
$$

where, $z$ represents the Gaussian random variable, $\mu$ is the mean of $z$ and $\sigma$ is the standard deviation of $z$. Figure 2-1 shows the probability density function for Gaussian noise. Approximately $68.27 \%$ of the values are found inside $\mu \pm \sigma, 95.45 \%$ of the values are found inside $\mu \pm 2 \sigma$ and $99.73 \%$ within $\mu \pm 3 \sigma$.

\subsection{Image denoising domains}

Image denoising can be performed either in the frequency domain or in the spatial domain. In case of frequency domain, an image is transformed into the frequency domain, the denoising operations are performed there, and the resulting denoised images are transformed back into the spatial domain. 


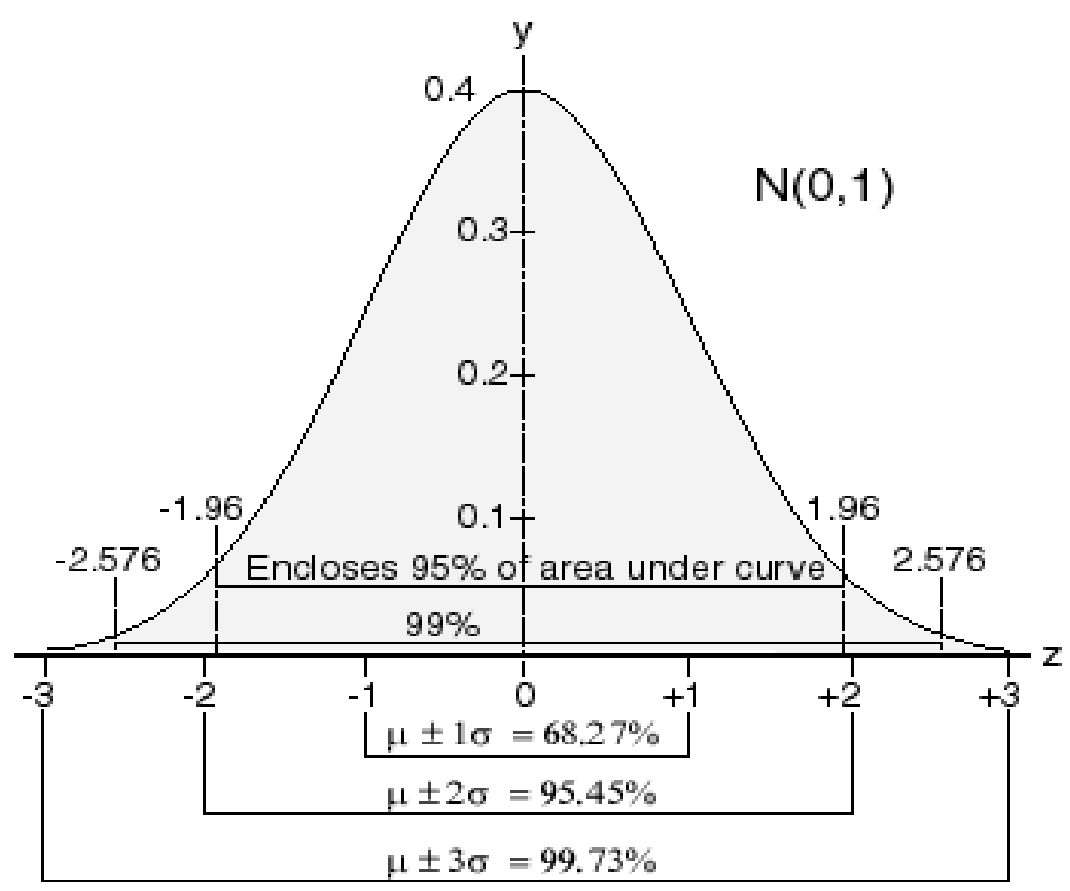

Figure 2-1: Probability Distribution for Gaussian noise

Perhaps the Block-Matching and 3D (BM3D) scheme [5] is one of the most successful image denoisign algorithms that operates in the frequency domain. It relies on the assumption that an image has a locally sparse representation in its transform domain. It attempts to find similar blocks with respect to a reference patch and builds a 3D stack of these $2 \mathrm{D}$ blocks. Then it applies 3D transform on the 3D stack and performs denoising. It then applies inverse 3D transform and return 2D estimate of the original image. Finally, collaborative filtering process gives a 3D estimation of the jointly filtered 2D blocks.

Spatial domain denoising works directly on the image data. One of the most successful spatial domain denoising scheme is the Non-Local Means algorithm. In the Non-Local Means algorithm a center pixel inside the reference patch is denoised by calculating a weighted average, where patches similar to the reference patch contribute into this averaging process. 


\subsection{Non-Local Means algorithm}

In the Non-Local Means algorithm a discrete noisy image $v=\{v(j) \mid j \in I\}$, where $I$ is the input image, can be denoised by the estimated value $\mathrm{NL}[\mathrm{v}](\mathrm{i})$ for a pixel $i$. It is computed as a weighted average for all of the pixels in the image,

$$
\mathrm{NL}[\mathrm{v}](\mathbf{i})=\sum_{\mathrm{j} \in \mathrm{I}} w(i, j) v(j)
$$

where, the weight $w(i, j)$ depends on the similarity between the pixel $i$ and the pixel $j$ of the intensity gray level vectors $v\left(N_{i}\right)$ and $v\left(N_{j}\right)$. Here, $N_{k}$ is the square patch around the center pixel $k$. The weight is then assigned to value $v(j)$ to denoise pixel $i$. The summation of all weight is equal 1 and each weight value $w(i, j)$ has a range between $[0,1]$. To measure similarity between patches, the Euclidean distance between patches is calculated

$$
\left\|v\left(N_{i}\right)-v\left(N_{j}\right)\right\|_{2}^{2}
$$

The weight $w(i, j)$ is computed as,

$$
w(i, j)=\frac{1}{Z(i)} e^{-\frac{\left\|v\left(N_{i}\right)-v\left(N_{j}\right)\right\|_{2}^{2}}{h^{2}}} .
$$

Here, $Z(i)$ is a normalization constant such that,

$$
\mathrm{Z}(i)=\sum_{\mathrm{j}} \mathrm{e}^{-\frac{\left\|v\left(N_{i}\right)-v\left(N_{j}\right)\right\|_{2, \sigma}^{2}}{h^{2}}}
$$

Here, $h$ is a smoothing kernel width which controls decay of the exponential function and therefore controls the decay of the weights as a function of the Euclidean distances.

The algorithm is summarized as follows, 


\section{Algorithm Non-Local Means}

Input I:Image with additive white Gaussian noise

\section{Output NL(I): Denoised image}

1. For each pixel $i$, where $i \in[1, N]$,

2. Do

2.1. For each pixel in $N_{k}$, where $N_{k}$ is the square patch around the center pixel $k$,

2.2. Do

Evaluate, normalization constant $\mathrm{Z}(i) \leftarrow \sum_{\mathrm{j}} \mathrm{e}^{-\frac{\left\|v\left(N_{i}\right)-v\left(N_{j}\right)\right\|_{2}^{2}}{h^{2}}}$, where $j$ refers to the $N_{k}$ patches.

2.2.2. $\quad$ Calculate, weight matrix $\mathrm{W}(\mathrm{i}, \mathrm{j}) \leftarrow \frac{1}{Z(i)} e^{-\frac{\left\|v\left(N_{i}\right)-v\left(N_{j}\right)\right\|_{2}^{2}}{h^{2}}}$

\subsubsection{Done}

2.3. $\quad$ Denoise pixel $i: \mathrm{NL}[\mathrm{v}](\mathrm{i}) \leftarrow \sum_{\mathrm{j} \in \mathrm{I}} w(i, j) v(j)$

\subsection{Done}

Figure 2-2 shows an example of the patch similarity measure for the NLM algorithm. Here, the reference patch $p$ is compared with its neighboring patches $q 1, q 2$ and $q 3$. As $q 1$ and $q 2$ are more similar to the reference patch $p$ than $q 3$, their weights, i.e. $w(p, q 1)$, $w(p, q 2)$, will be higher than $q 3$ weight, i.e. $w(p, q 3)$. 


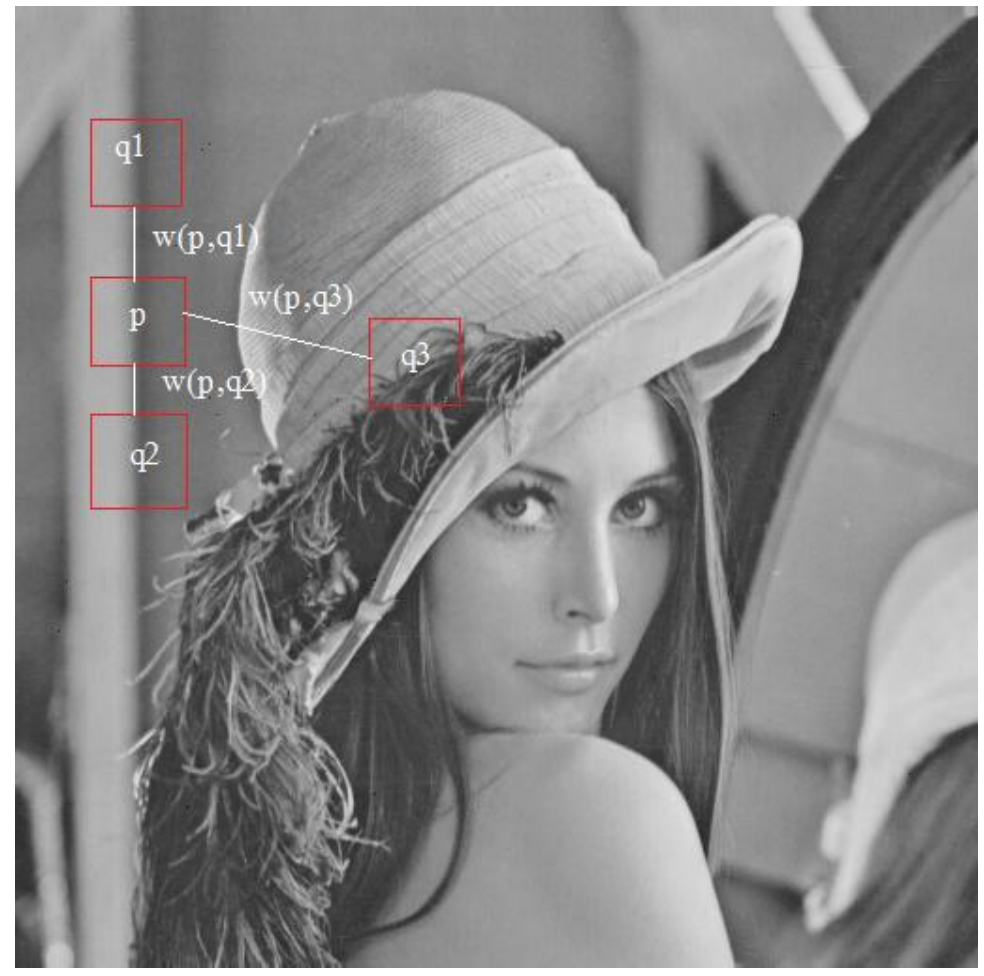

Figure 2-2: The Non-Local Means scheme where similar patches $q 1$ and $q 2$ are assigned weights larger than $q 3$.

In the NLM algorithm, when a patch size is $M \times M$, the search region size is $p \times p$, and the image size is $K \times K$, the complexity of the NLM algorithm will be $O\left(p^{2} M^{2} K^{2}\right)$.

\subsection{Applications of Non-Local Means}

The Non-Local Means algorithm has been used in many applications. It has been used in medical imaging such as on MR brain image [14][15], CT scan image [16], 3D ultrasound imaging [17][18], diagnosis of heart echo images [19] . It has been used in other applications such as video denoising [20][21][22], SAR image denoising [23][24], surface salinity detection [25][26], and metal artifact detection [27]. 


\subsection{Improvement over Non-Local Means}

Many improvements have been suggested on the Non-Local Means algorithm in recent years. Most of the significant improvements on the Non-Local Means algorithm have been done using the patch regression, probabilistic early termination, a patch based dictionary, neighborhood classification, principal component analysis and cluster trees. In this section, we have described them briefly.

Bhujle [9] proposed a dictionary based denoising in which patches with similar photometric structures are clustered together to create groups. Here, they build a dictionary prior to denoising which can be accessed at a constant time. In their proposed method, they build a global dictionary from all test images. Their approach can find almost similar patches from the global dictionary in a short amount of time instead of searching around the whole search window.

To search inside the dictionary, they build a tree data structure where searching starts from the root node and calculate the distance between the reference patch. They also suggested another improvement related to the patch edges. It improves the space and time complexity by storing the residual image into the dictionary.

The proposed dictionary based NLM and their improvements on edge patch based dictionary outperforms the original NLM by preselecting the similar patches and performs denoising based on the calculated weights. In addition, edge patch based dictionary reduces space and time to perform denoising by preselecting similar patches based on residual edge image.

Mahmoudi et al. [10] accelerate the NLM algorithm by pre-classifying neighborhood patches based on average gray values, gradient orientation, or both.

Chaudhury et al. [6] claimed that the denoising performance of the Non-Local Means algorithm can be improved by replacing the mean operation by a median operation. 
Vignesh et al. [7] proposed a speed up technique for the Non-Local Means algorithm based on a probabilistic early termination (PET). In the original Non-Local Means algorithm [4] distance calculation takes a significant amount of time. Neighborhood selection can be done earlier using a soft decision. Contributing pixels can be rejected when the expected distance value is below the weighted average. Probability models based on patch features are used at each stage of distance computation to accept or reject a patch. This scheme is called the probabilistic early termination (PET) scheme.

Tasdizen et al. [12] proposed principal component based Non-Local Means algorithm where a global feature space is created to select important features. Image patches are projected into the lower dimensional feature space and the dimensionality is reduced. This reduced feature space is used for similarity measure rather than the entire feature space.

Here, they proposed PCA to reduce the dimensionality of this feature space. PCA is applied on the global feature space rather than on local feature space to provide an efficient algorithm. They sort the eigenvectors in a descending order of eigen values and projected the image patches into the lower subspace.

Reduced feature space gives better results over the original NLM denoising algorithm and the author claims that it performs better in all cases. PCA is a data driven approach and can adapt to a given image.

Brox et al. [13] proposed a technique to improve the performance of the NLM method using a clustering tree. Here they introduced two novel techniques for NLM. Firstly, they have introduced clustering tree for the Non-Local Means algorithm which allows a fast pre-selection. It performs faster when the NLM algorithm considers the whole image as a search region and works better for a fixed window size. Secondly, they have introduced an iterative version of the filter to perform better in regular and textured images. 


\subsection{T-test}

A hypothesis is a statement or claim about the state of an incident e.g. state of nature, scientific investigation, market analysis, weather prediction which is unknown. Statistical hypothesis is stated in terms of population parameters e.g. population mean and variance. Researchers gather data and look for evidence to support or contradict about it. Testing a hypothesis refers to accumulating relevant information and making a decision about the action to be taken about the hypothesis. Testing a statistical hypothesis involves (1) determination of test statistics and (2) utilization of the sample values of the statistics. The test is performed to chose either a given hypothesis (called null hypothesis $H_{0}$ ) or a competing hypothesis (called alternate hypothesis $H_{1}$ ).

Let, testing procedure comprises that a statistics is a function of several random variables $X_{1}, X_{2}, \ldots, X_{n}$ which gives,

$$
V=V\left(X_{1}, X_{2}, \ldots, X_{n}\right)
$$

Based on the observed random samples $\mathrm{V}$, it decides to choose hypothesis between Ho or $H$. In respect to the distribution of $V$, two regions are chosen. Accepted region consists of the values who adopt null hypothesis Ho. Rejected regions adopt alternate hypothesis $H_{1}$. Here the main terminology is to decide whether a null hypothesis Ho is accepted or rejected.

The population random variable $X$ is a part of the competing hypotheses and their distribution is not fully known. The observation of $\mathrm{V}$ leads to a decision regarding the chosen hypothesis. For example, if a random variable contains a parameter $\theta$, which may have two observation $\theta_{0}$ or $\theta_{1}$, The test statistics helps to decide whether to accept or reject $\theta$. For $\theta_{0}$ as null hypothesis, we can write the following equations to represent hypothesis testing problem.

$$
\begin{aligned}
& H_{o}: \theta=\theta_{0} \\
& H_{1}: \theta=\theta_{1}
\end{aligned}
$$


Possible outcome of the test is divided into two classes. One in the acceptance region A and other in the critical region or rejection region B. Finally, if $\mathrm{V}$ falls in region $\mathrm{A}$ then $H_{o}$ is accepted and if it falls in B then $H_{o}$ is rejected.

It can be well explained using an example. Suppose that there are two identical boxes of jelly beans. Box 1 contains 60 red jelly beans and 40 green jelly beans. Box 2 contains 40 red jelly beans and 60 green jelly beans. The proportion of red jelly beans $p$, for these two boxes are

Box 1: $\mathrm{p}=0.60$

Box 2: $\mathrm{p}=0.40$

Suppose that there is a box on a table, but we do not know which one it is. We assume that it is box 2, but we are not sure about that. To test our hypothesis that the box 2 is on the table or not, we pick 10 random jelly beans. The number of red jelly beans in the test samples will be used to decide whether box 2 is on the table or not.

W.S. Gosset derived the probability distribution for this statistics which named as "student's t" or simply $t$ distribution. Figure 2-3 shows the student $t$ distribution. This function has only one parameter named degree of freedom. That is why $t$ distribution with $v$ degree of freedom is called $t(v)$ which is quite similar to the normal distribution having bell shaped curve. The difference between normal distribution and $t$ distribution is that $t$ distribution has a fatter tail over the normal distribution. This concluded as $t$ distribution has more probability in the extreme tail over the normal distribution. This characteristic persists as for small value of the degree of freedom but it reduces as degree of freedom exceeds 30 or more. When the degree of freedom is infinite, $t$ distribution is identical to standard normal distribution. 


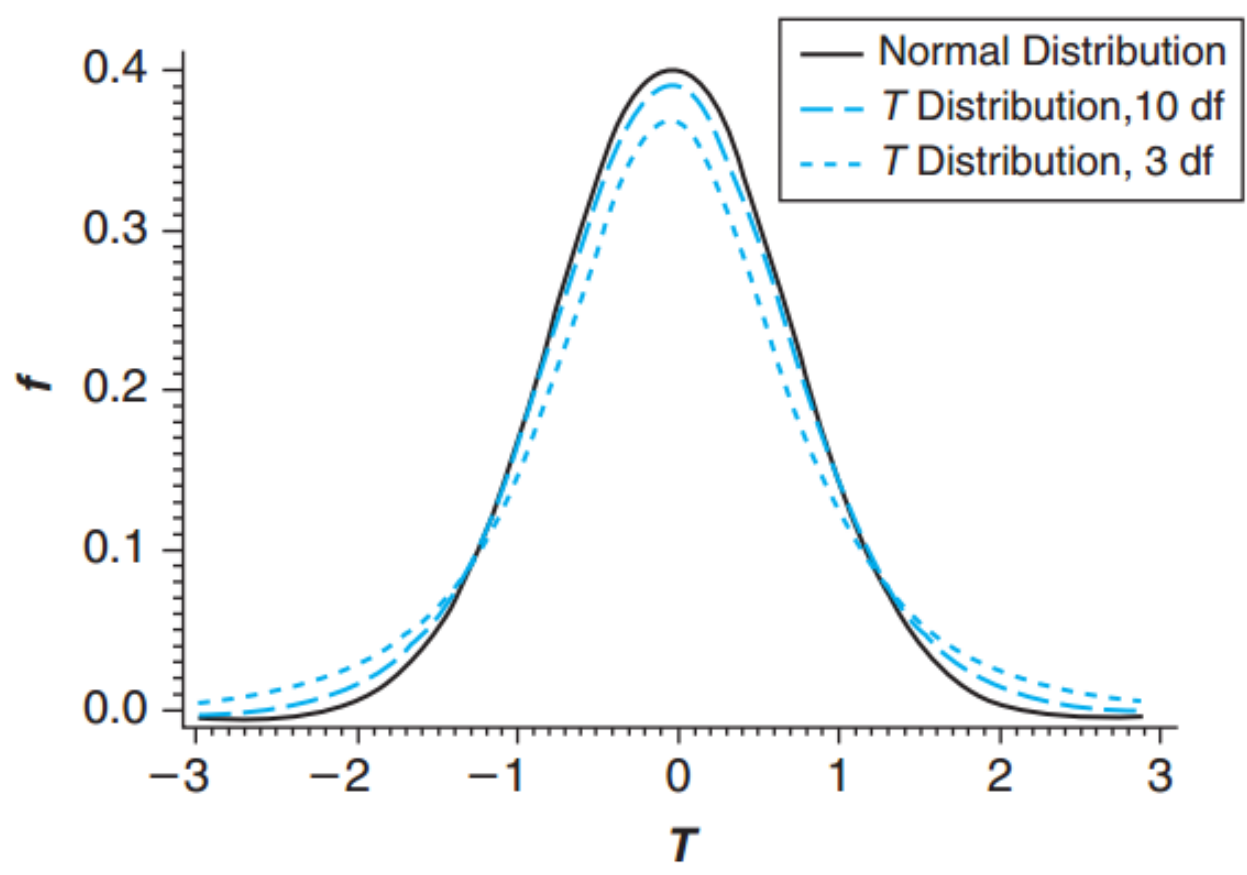

Figure 2-3: Student t distribution

In summary, we have discussed Gaussian noise, image denoising domains, the Non-Local Means algorithm, as well as its variants. In addition we have discussed statistical $t$-test in this chapter. 


\section{Chapter 3}

\section{Methodology}

Non-Local Means is one of the most popular and powerful image denoising algorithm available in recent years. It performs denoising in spatial domain and improves visual quality of a noisy image. It can preserve edges and fine details. It denoises the center pixel inside a reference patch by calculating a weighted average. Patches similar to the reference patch contribute into this averaging process. In our thesis, we have reduced the computational time to find similar patches by reducing the feature space.

\subsection{Improved Non-Local Means Algorithm}

Non-Local Means algorithm needs to search its neighboring area to find similar patches. The utilized image patch size is usually $5 \times 5,7 \times 7$ or $9 \times 9$, which can be represented by 25 , 49 or 81 dimensional feature vectors, respectively. This feature vector space is used to assess the similarity between patches. In our proposed algorithm, a global feature vector space is created in a preprocessing step (step 1). After that, a statistical test called t-test is performed on this global feature vector space to reduce its dimensionality (step 2). This reduced feature vector space is used during the rest of the denoising process.

\subsubsection{Preprocessing}

In the first step, we have created a feature vector space for the noisy image. An image patch is linearized and represented as a row vector of size $j$. Thus the dimension of this feature vector space will be $j \times N$, where $N$ is the total number of pixels in an image. Feature vectors can be represented as matrix $\mathrm{C}$, 


$$
C=\left[\begin{array}{cccc}
c(1,1) & c(1,2) & \ldots & c(1, j) \\
c(2,1) & c(2,2) & \ldots & c(2, j) \\
\ldots & \ldots & \ldots & \ldots \\
c(N, 1) & c(N, 2) & \ldots & c(N, j)
\end{array}\right]
$$

Here, for example if we have a patch size of $7 \times 7$ then $j$ will be equal to 49 . This matrix will be used during the dimensionality reduction process.

\subsubsection{T-test}

We have implemented a paired $t$-test of the null hypothesis. This test is performed on the matrix C. For each test case (i.e., each column in the matrix C), once the $t$ value is determined, the students t-distribution lookup table is used to find the value of $p$. When the calculated $\mathrm{p}$ value is below a given threshold value, then the null hypothesis is rejected. In our denoising problem, we have considered each patch as a feature vector. The hypothesis tries to accept or reject a feature (i.e. an entire column in the matrix C). Here, the null hypothesis is whether a feature is significant or not. In calculating the null hypothesis, one uses the following normalization equation

$$
T=\frac{\bar{x}-\mu_{0}}{s / \sqrt{n}}
$$

Where, $\bar{x}$ is the sample mean, $\mu_{0}$ is the population mean, $s$ is the sample standard deviation and $n$ is the sample size. When the null hypothesis is accepted, it concludes that the feature is significant. Otherwise, this feature is not significant. Thus the entire column is deleted and hence reduces the size of matrix $\mathrm{C}$. 


\subsubsection{Non-Local Means algorithm}

In the Non-Local Means algorithm, a discrete noisy image $v=\{v(i) \mid i \epsilon I\}$, where $I$ is the input image, can be denoised by calculating the weighted average,

$$
N L[v](i)=\sum_{j \epsilon I} w(i, j) v(j)
$$

Here, the weight $w(i, j)$ depends on the similarity between the pixel $i$ and the pixel $j$ of the intensity gray level vectors $v\left(N_{i}\right)$ and $v\left(N_{j}\right)$. Here, $N_{k}$ is the square patch around the center pixel. This weight is assigned to value $v(j)$ which denoises pixel $i$. It can be computed as,

$$
w(i, j)=\frac{1}{Z(i)} e^{-\frac{\left\|v\left(N_{i}\right)-v\left(N_{j}\right)\right\|_{2}^{2}}{h^{2}}}
$$

Here, $h$ is the smoothing kernel width which controls the decay of the exponential

function. $\left\|v\left(N_{i}\right)-v\left(N_{j}\right)\right\|_{2}^{2}$ is the Euclidean distance between two pixels $i$ and $j . Z(i)$ is a normalization constant calculated as,

$$
\mathrm{Z}(i)=\sum_{\mathrm{j}} \mathrm{e}^{-\frac{\left\|v\left(N_{i}\right)-v\left(N_{j}\right)\right\|_{2}^{2}}{h^{2}}}
$$

We have reduced the size of the feature vector over the original NLM algorithm. In our proposed method $N_{k}$ is replaced by $f_{k}$, where $f_{k}$ is the reduced feature vector. Then we have selected similar patches and calculated weights based on this reduced feature vector. 
Our proposed algorithm is summarized as follows.

\section{Algorithm Improved Non-Local Means}

Input I:Image with additive white Gaussian noise

Output NL(I): Denoised image

1. Crate a global feature matrix $C$ (as shown in Equation 3.1).

2. Perform the $t$-test on matrix $C$ to produce the reduced row matrix $f_{k}$.

3. For each pixel $i$, where $i \in[1, N]$,

4. Do

4.1. For each pixel in $N_{k}$, where $N_{k}$ is the square patches around the center pixel $k$,

4.2. Do

4.2.1. $\quad$ Evaluate the normalization constant $\mathrm{Z}(i) \leftarrow \sum_{\mathrm{j}} \mathrm{e}^{-\frac{\left\|v\left(f_{i}\right)-v\left(f_{j}\right)\right\|_{2}^{2}}{h^{2}}}$, where $j$ refers to the $N_{k}$ patches.

4.2.2. $\quad$ Calculate the weight matrix $\mathrm{W}(\mathrm{i}, \mathrm{j}) \leftarrow \frac{1}{Z(i)} e^{-\frac{\left\|v\left(f_{i}\right)-v\left(f_{j}\right)\right\|_{2}^{2}}{h^{2}}}$

\subsubsection{Done}

4.3. $\quad$ Denoise pixel $i: \operatorname{NL}[\mathrm{v}](\mathrm{i}) \leftarrow \sum_{\mathrm{j} \in \mathrm{I}} w(i, j) v(j)$

\subsection{Done}




\subsubsection{Parameter Setting}

Our proposed algorithm depends on the following parameters,

1. Patch size,

2. Search region size,

3. Threshold value.

We have analyzed the effect of these parameters on our test images and reported their comparative performance in terms of PSNR (see Section 4.3.1) in the following sections. Figure 3-1 shows the test images used in our experiment.

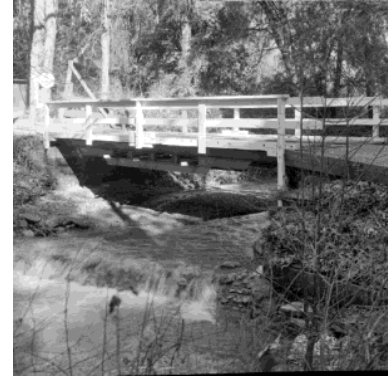

(a) Bridge

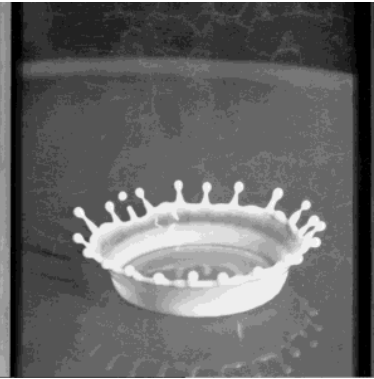

(e) Milk drop

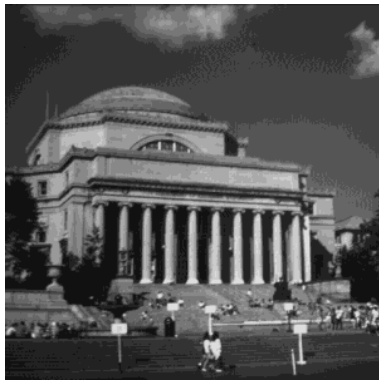

(b) Columbia

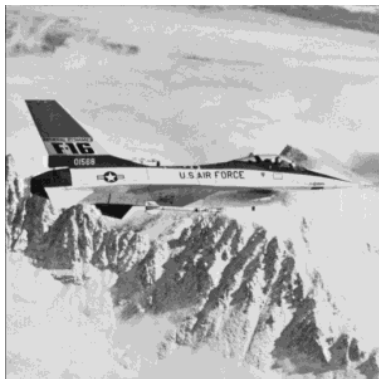

(f) Plane

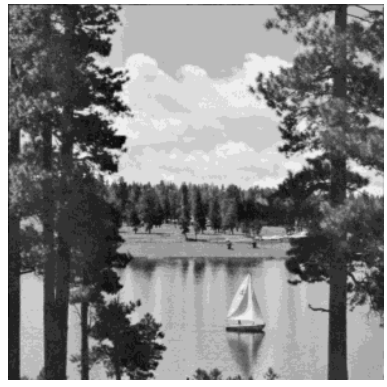

(c) Lake

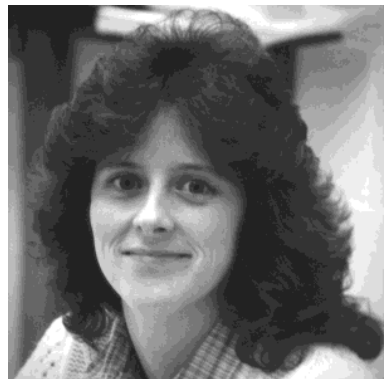

(g) Woman 1

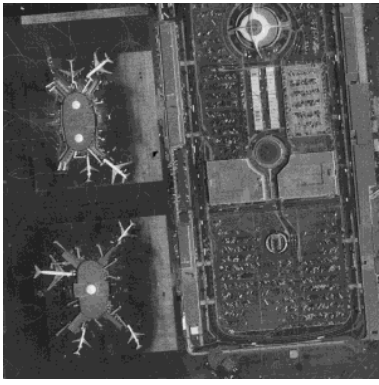

(d) Lax

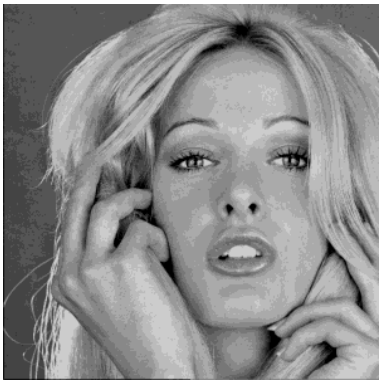

(h) Woman 2

Figure 3 - 1: Set of test images $(512 \times 512)$ for performance analysis. 


\subsubsection{Patch Size}

Large patch size suppresses small details whereas small patch size fails to denoise properly. Yet in the case of large patch size, it is difficult to find patches similar to the reference patch, as such repeated patterns may appear less frequently.

In our experiment we have three parameters. Here, we have fixed the size of search region and the threshold value. We have taken the search region of size $21 \times 21$ and the threshold value 5. Then we have analyzed the effect of different patch sizes on our test images at various noise levels.

Table 3-1 shows the effect of various patch sizes on our test images and reported their average PSNR values over all test images at various noise levels. It has been found that, patch size $7 \times 7$ works better for noise level $\sigma<80$ and patch size $5 \times 5$ works better for higher noise levels.

Figure 3-2(a) - (d) show the effect of patch sizes $5 \times 5,7 \times 7,9 \times 9$ and $11 \times 11$, respectively on Woman 1 image for noise level $\sigma=40$. It has been found that Figure 3-2 (b) performs better over all other produced images. 
Table 3- 1: Average PSNR comparison for all test images between different patch sizes for different noise levels.

\begin{tabular}{|c|c|c|c|c|c|}
\hline Noise Level & $3 \times 3$ & $5 \times 5$ & $7 \times 7$ & $9 \times 9$ & $11 \times 11$ \\
\hline \multicolumn{6}{|c|}{ Search region sizes $21 \times 21$, threshold value $=5$} \\
\hline 10 & 32.10 & 33.62 & 33.75 & 32.93 & 32.08 \\
\hline 20 & 29.25 & 30.42 & 30.60 & 29.88 & 29.07 \\
\hline 30 & 27.51 & 28.67 & 28.78 & 28.09 & 27.27 \\
\hline 40 & 26.29 & 27.33 & 27.49 & 26.83 & 25.91 \\
\hline 50 & 25.42 & 26.42 & 26.54 & 25.94 & 25.04 \\
\hline 60 & 23.18 & 24.11 & 24.27 & 23.67 & 23.10 \\
\hline 70 & 21.93 & 22.99 & 23.03 & 22.45 & 22.08 \\
\hline 80 & 21.49 & 22.52 & 22.25 & 21.94 & 21.52 \\
\hline 90 & 20.78 & 21.76 & 21.74 & 21.09 & 20.85 \\
\hline 100 & 20.14 & 21.12 & 21.07 & 20.59 & 20.28 \\
\hline
\end{tabular}




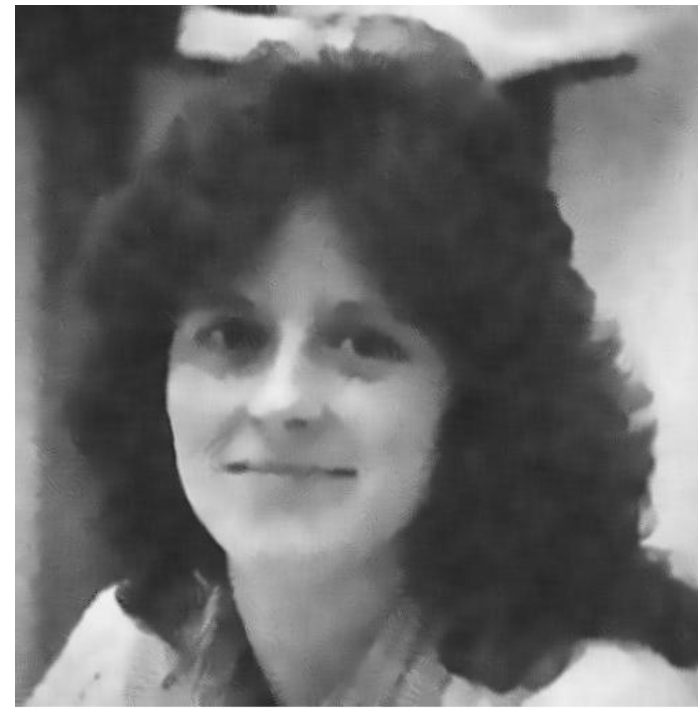

(a)

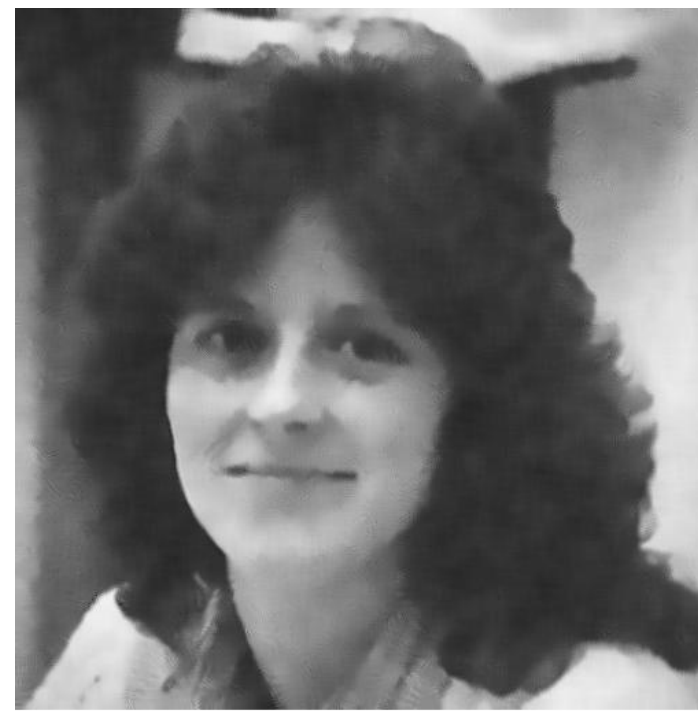

(c)

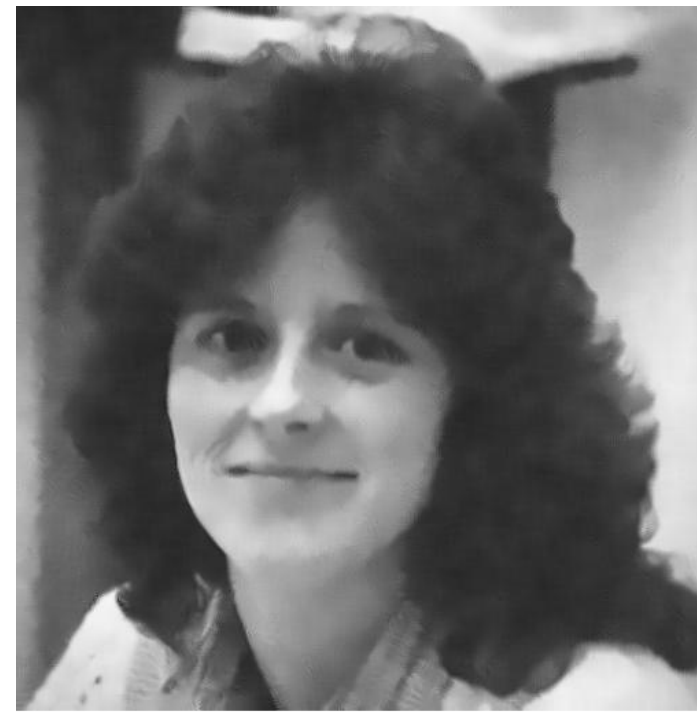

(b)

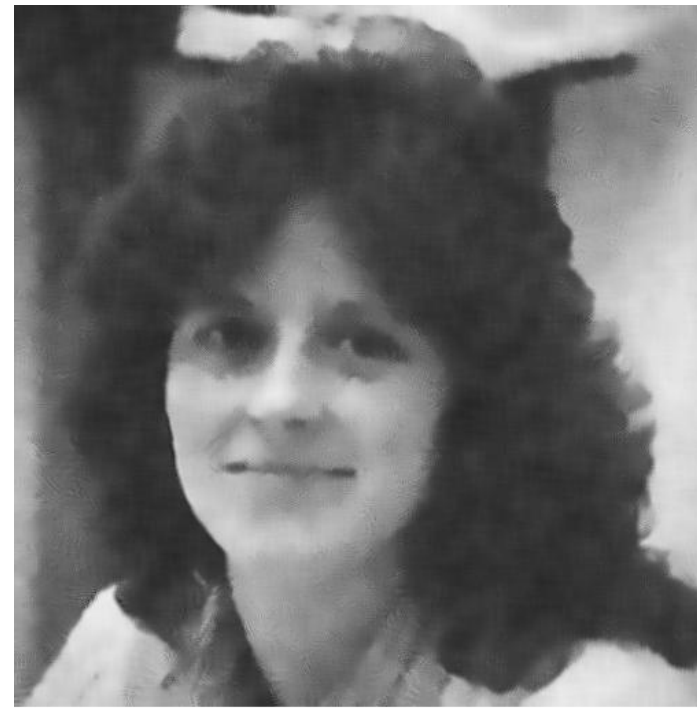

(d)

Figure 3 - 2: Performance analysis using Woman 1 image over different patch sizes where noise level is $\sigma=40$. (a) patch size $5 \times 5$, PSNR $=29.55$, (b) patch size $7 \times 7$, PSNR $=$ 29.79, (c) patch size 9×9, PSNR = 29.31, and (d) patch size $11 \times 11$, PSNR $=28.10$. 


\subsubsection{Search Region Size}

Large search region size helps to find more patches similar to the reference patch. Whereas, small search region size faces difficulty to find enough similar patches.

Here, we have fixed the size of the threshold value. We have taken the patch sizes from Section 3.1.4.1. For noise level $\sigma<80$, we have chosen patch size $7 \times 7$ and the threshold value 5 . For noise level $\sigma>80$, we have chosen patch size $5 \times 5$ and the threshold value 5 . Then we have observed the effect of different search region sizes over all test images at various noise levels.

Table 3-2 shows the effect of search region sizes on our test images and reported their average PSNR values. It has been found that, for patch size $7 \times 7$ and noise level $\sigma<80$, the search region sizes $21 \times 21,28 \times 28$ and $35 \times 35$ perform the best. For patch size $5 \times 5$ and noise level $\sigma>80$, the search region sizes $15 \times 15,20 \times 20$ and $25 \times 25$ show the best performance. We have selected the minimum search region size for each case to reduce complexity.

Figure 3-3 (a)-(d) show the effect of search region sizes $14 \times 14,21 \times 21,28 \times 28$ and $35 \times 35$, respectively on Woman 1 image for noise level $\sigma=40$. Figure 3-3 (b), (c) and (d) show almost similar performance and Figure 3-3(a) shows the worst performance. 
Table 3- 2: Average PSNR comparison for all test images between different search region sizes for different noise levels.

\begin{tabular}{|c|c|c|c|c|}
\hline Noise Level & $14 \times 14$ & $21 \times 21$ & $28 \times 28$ & $35 \times 35$ \\
\hline \multicolumn{5}{|c|}{ Patch size $7 \times 7$, threshold value $=5$} \\
\hline 10 & 32.12 & 33.75 & 33.75 & 33.75 \\
\hline 20 & 29.34 & 30.60 & 30.60 & 30.61 \\
\hline 30 & 27.60 & 28.78 & 28.78 & 28.78 \\
\hline 40 & 26.37 & 27.49 & 27.49 & 27.49 \\
\hline 50 & 25.47 & 26.54 & 26.54 & 26.55 \\
\hline 60 & 23.26 & 24.27 & 24.27 & 24.27 \\
\hline 70 & 22.08 & 23.03 & 23.03 & 23.04 \\
\hline Noise Level & $10 \times 10$ & $15 \times 15$ & $20 \times 20$ & $25 \times 25$ \\
\hline \multicolumn{5}{|c|}{ Patch size $5 \times 5$, threshold value $=5$} \\
\hline 80 & 21.45 & 22.52 & 22.52 & 22.52 \\
\hline 90 & 20.86 & 21.75 & 21.75 & 21.76 \\
\hline 100 & 20.25 & 21.12 & 21.12 & 21.12 \\
\hline
\end{tabular}




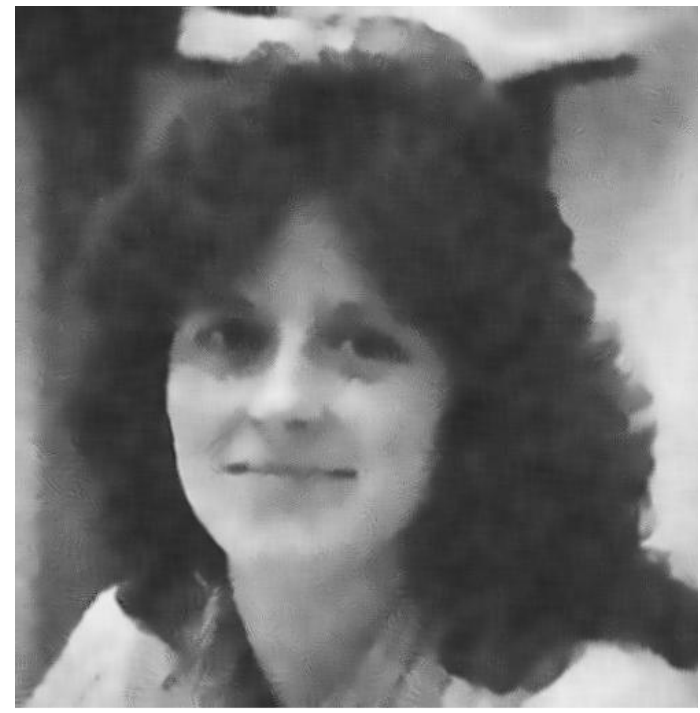

(a)

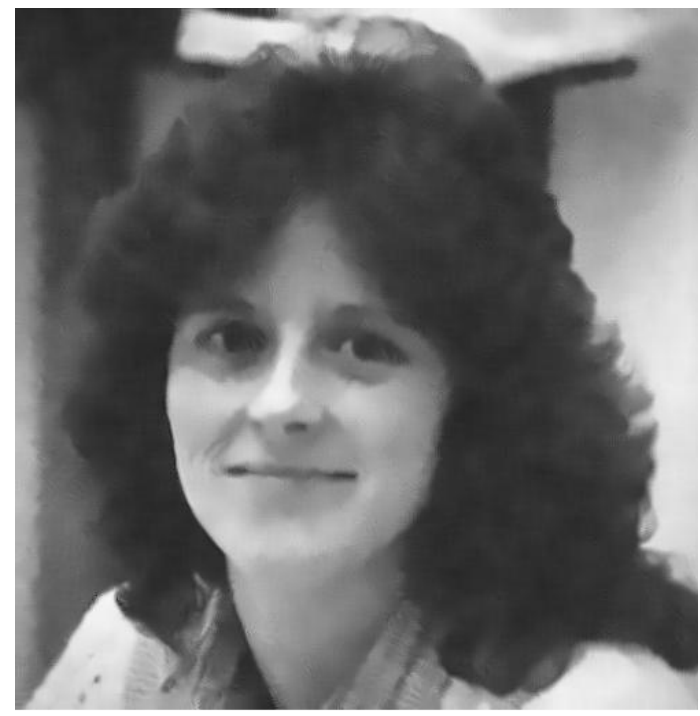

(c)

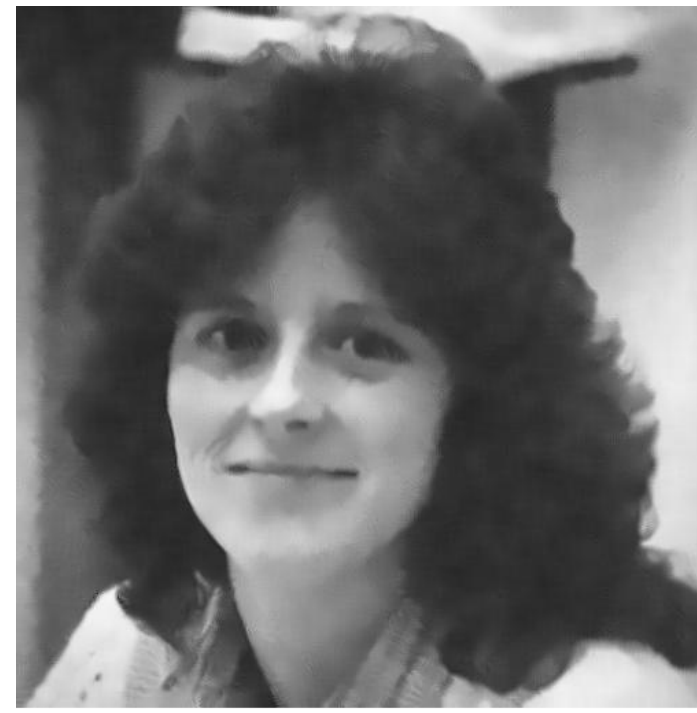

(b)

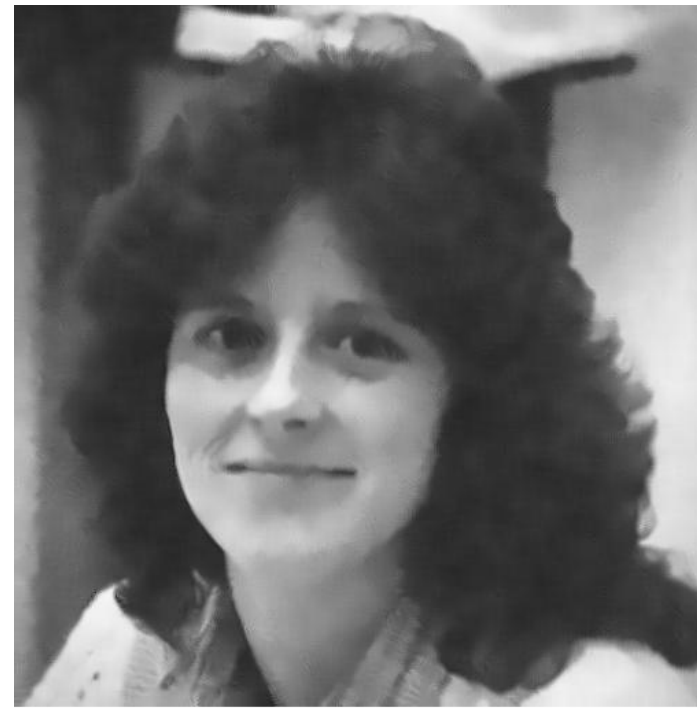

(d)

Figure 3 - 3: Performance analysis using Woman 1 image over different search region sizes where noise level is $\sigma=40$ (a) search region size $=14 \times 14, \operatorname{PSNR}=29.11$, (b) search region size $=21 \times 21$, PSNR $=29.78$, (c) search region size $=28 \times 28$, PSNR $=29.78$, and $(d)$ search region size $=35 \times 35, \mathrm{PSNR}=29.79$. 


\subsubsection{Threshold value}

The threshold value determines whether a hypothesis is accepted or rejected. Here, we have analyzed the effect of this threshold value for different noise levels.

Here, we have chosen parameters form the Section 3.1.4.1 and Section 3.1.4.2. For a noise level $\sigma<80$, we have chosen the patch size $7 \times 7$ and the search region size $21 \times 21$. For noise level $\sigma>80$, we have chosen the patch size $5 \times 5$ and the search region size $15 \times 15$. Then we have observed the effect of different threshold values over all test images at various noise levels.

Table 3-3 and Table 3-4 show the value of average PSNR and the average number of features selected for each noise levels at various threshold values. Here, the threshold value represents the percentage of rejection. The bolded PSNR values represent the best results among these two tables. If we check the values from these two tables, we can find that the threshold value 3 and the threshold value 5 show the best result for the noise levels $\sigma<80$, patch size of $7 \times 7$ and for the noise levels $\sigma>80$, patch size of $5 \times 5$, respectively. We have chosen threshold value 5 as it requires fewer features to produce the same result.

Figure 3-4 and Figure 3-5 show the average PSNR and the average number of features for the patch size $7 \times 7$ for the threshold value 3 and the threshold value 5 , respectively over all test images at various noise levels. Figure 3-6 and Figure 3-7 show the average PSNR and the average number of features for the patch size $5 \times 5$ for threshold value 3 and the threshold value 5, respectively. Figure 3-8 (a)-(d) show the effect of the threshold values at 3,5,7 and 9, respectively on Woman 1 image for noise level $\sigma=40$. It has been found that 3 and 5 work batter over others. 
Table 3- 3: Average PSNR and average number of features comparison for patch size $7 \times 7$ for all test images between different threshold values for different noise levels.

\begin{tabular}{|c|c|c|c|c|c|c|}
\hline Noise Level & & 3 & 5 & 7 & 9 & 11 \\
\hline \multicolumn{7}{|c|}{ Patch size $7 \times 7$, search region size $21 \times 21$} \\
\hline \multirow{2}{*}{10} & PSNR & 33.75 & 33.75 & 33.51 & 33.31 & 33.12 \\
\hline & \# of Features & 42.13 & 39.38 & 36.88 & 35.38 & 33.63 \\
\hline \multirow{2}{*}{20} & PSNR & 30.60 & 30.60 & 30.39 & 30.21 & 30.02 \\
\hline & \# of Features & 40.38 & 38.13 & 35.75 & 33.88 & 32.75 \\
\hline \multirow{2}{*}{30} & PSNR & 28.79 & 28.78 & 28.54 & 28.41 & 28.12 \\
\hline & \# of Features & 39.75 & 37.13 & 35.75 & 33.63 & 31.75 \\
\hline \multirow{2}{*}{40} & PSNR & 27.48 & 27.48 & 27.30 & 27.24 & 27.10 \\
\hline & \# of Features & 39.13 & 36.88 & 34.63 & 32.76 & 30.75 \\
\hline \multirow{2}{*}{50} & PSNR & 26.54 & 26.54 & 26.33 & 26.25 & 26.10 \\
\hline & \# of Features & 38.5 & 36 & 33.89 & 32.63 & 30.63 \\
\hline \multirow{2}{*}{60} & PSNR & 24.27 & 24.27 & 24.11 & 23.95 & 23.85 \\
\hline & \# of Features & 37.75 & 35.38 & 33 & 32.5 & 30.38 \\
\hline \multirow{2}{*}{70} & PSNR & 23.03 & 23.03 & 22.94 & 22.85 & 22.69 \\
\hline & \# of Features & 37.63 & 35.73 & 32.88 & 31.75 & 29.5 \\
\hline \multirow{2}{*}{80} & PSNR & 22.26 & 22.26 & 22.24 & 22.18 & 22.11 \\
\hline & \# of Features & 36.88 & 33.87 & 31 & 29.88 & 28.5 \\
\hline \multirow{2}{*}{90} & PSNR & 21.74 & 21.74 & 21.71 & 21.66 & 21.58 \\
\hline & \# of Features & 36.5 & 33.88 & 30.88 & 29.75 & 28.38 \\
\hline \multirow{2}{*}{100} & PSNR & 21.07 & 21.06 & 21.08 & 20.98 & 20.96 \\
\hline & \# of Features & 35.88 & 33 & 30 & 28.88 & 27.63 \\
\hline
\end{tabular}


Table 3- 4: Average PSNR and average number of features comparison for patch size $5 \times 5$ for all test images between different threshold values for different noise levels.

\begin{tabular}{|c|c|c|c|c|c|c|}
\hline Noise Level & & 3 & 5 & 7 & 9 & 11 \\
\hline \multicolumn{7}{|c|}{ Patch size $5 \times 5$, search region size $15 \times 15$} \\
\hline \multirow{2}{*}{10} & PSNR & 33.61 & 33.61 & 33.57 & 33.56 & 33.55 \\
\hline & \# of Features & 24.17 & 23.5 & 23.17 & 22.83 & 22.17 \\
\hline \multirow{2}{*}{20} & PSNR & 30.38 & 30.37 & 30.36 & 30.33 & 30.31 \\
\hline & \# of Features & 24.17 & 23.5 & 22.83 & 22.67 & 21.83 \\
\hline \multirow{2}{*}{30} & PSNR & 28.65 & 28.64 & 28.61 & 28.60 & 28.59 \\
\hline & \# of Features & 23.83 & 23 & 22.67 & 22.5 & 21.83 \\
\hline \multirow{2}{*}{40} & PSNR & 27.32 & 27.31 & 27.31 & 27.30 & 27.29 \\
\hline & \# of Features & 23.83 & 22.83 & 21.83 & 21.67 & 20.83 \\
\hline \multirow{2}{*}{50} & PSNR & 26.41 & 26.41 & 26.40 & 26.39 & 26.38 \\
\hline & \# of Features & 23 & 22.67 & 21.83 & 21 & 20.83 \\
\hline \multirow{2}{*}{60} & PSNR & 24.10 & 24.10 & 24.09 & 24.08 & 24.07 \\
\hline & \# of Features & 23 & 21.83 & 21 & 20 & 19.67 \\
\hline \multirow{2}{*}{70} & PSNR & 22.98 & 22.97 & 22.97 & 22.96 & 22.95 \\
\hline & \# of Features & 22.67 & 21.83 & 20.83 & 19.17 & 18.83 \\
\hline \multirow{2}{*}{80} & PSNR & 22.52 & 22.52 & 22.33 & 22.15 & 21.94 \\
\hline & \# of Features & 22.67 & 21 & 19 & 19 & 17.83 \\
\hline \multirow{2}{*}{90} & PSNR & 21.76 & 21.76 & 21.50 & 21.34 & 21.11 \\
\hline & \# of Features & 21.83 & 20.83 & 19 & 18 & 17.33 \\
\hline \multirow{2}{*}{100} & PSNR & 21.12 & 21.12 & 20.91 & 20.81 & 20.73 \\
\hline & \# of Features & 21.83 & 20.5 & 18.5 & 17.83 & 17 \\
\hline
\end{tabular}




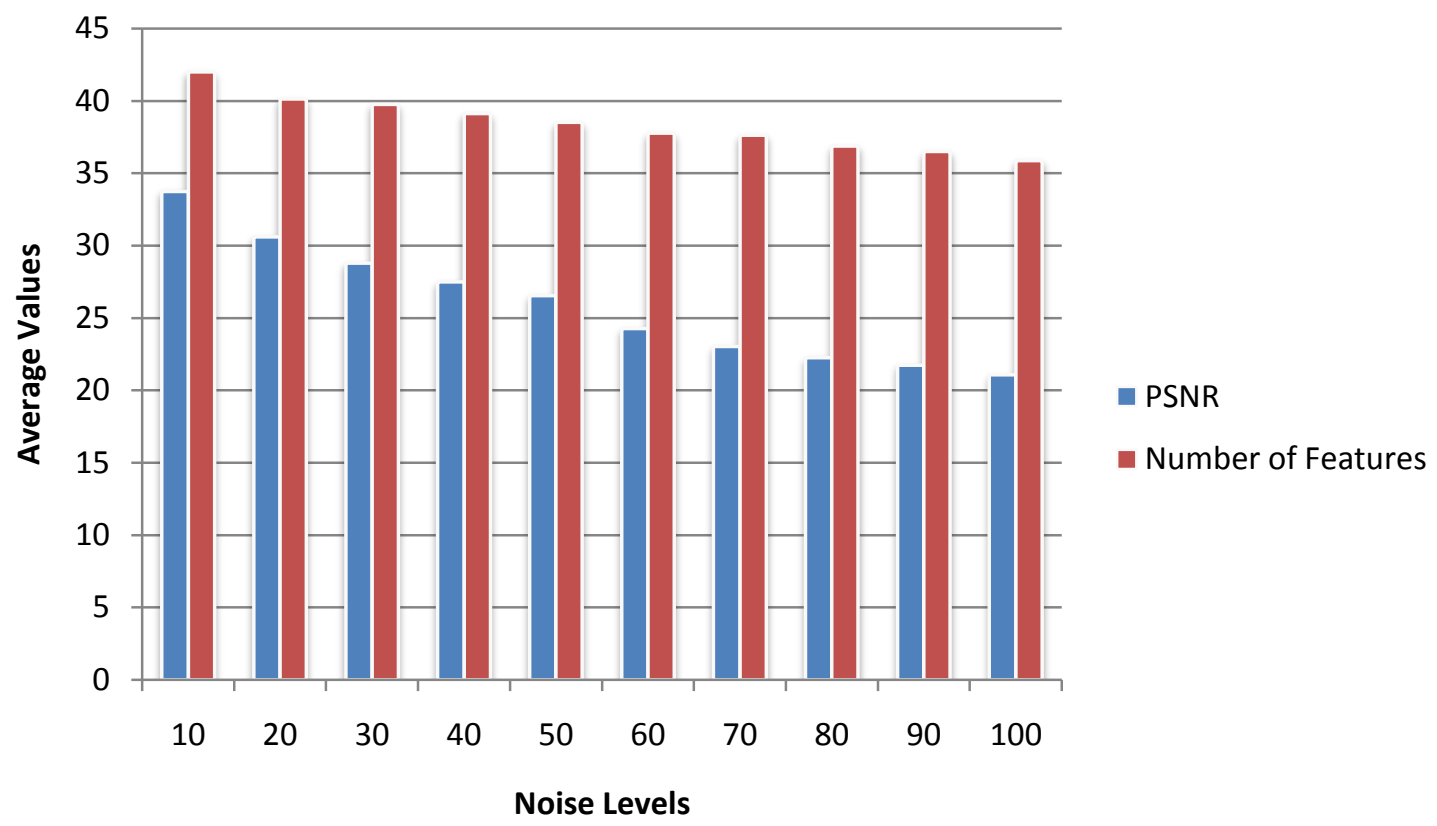

Figure 3 - 4: Average PSNR and average number of features comparison for patch size $7 \times 7$ and threshold value 3 over different noise levels.

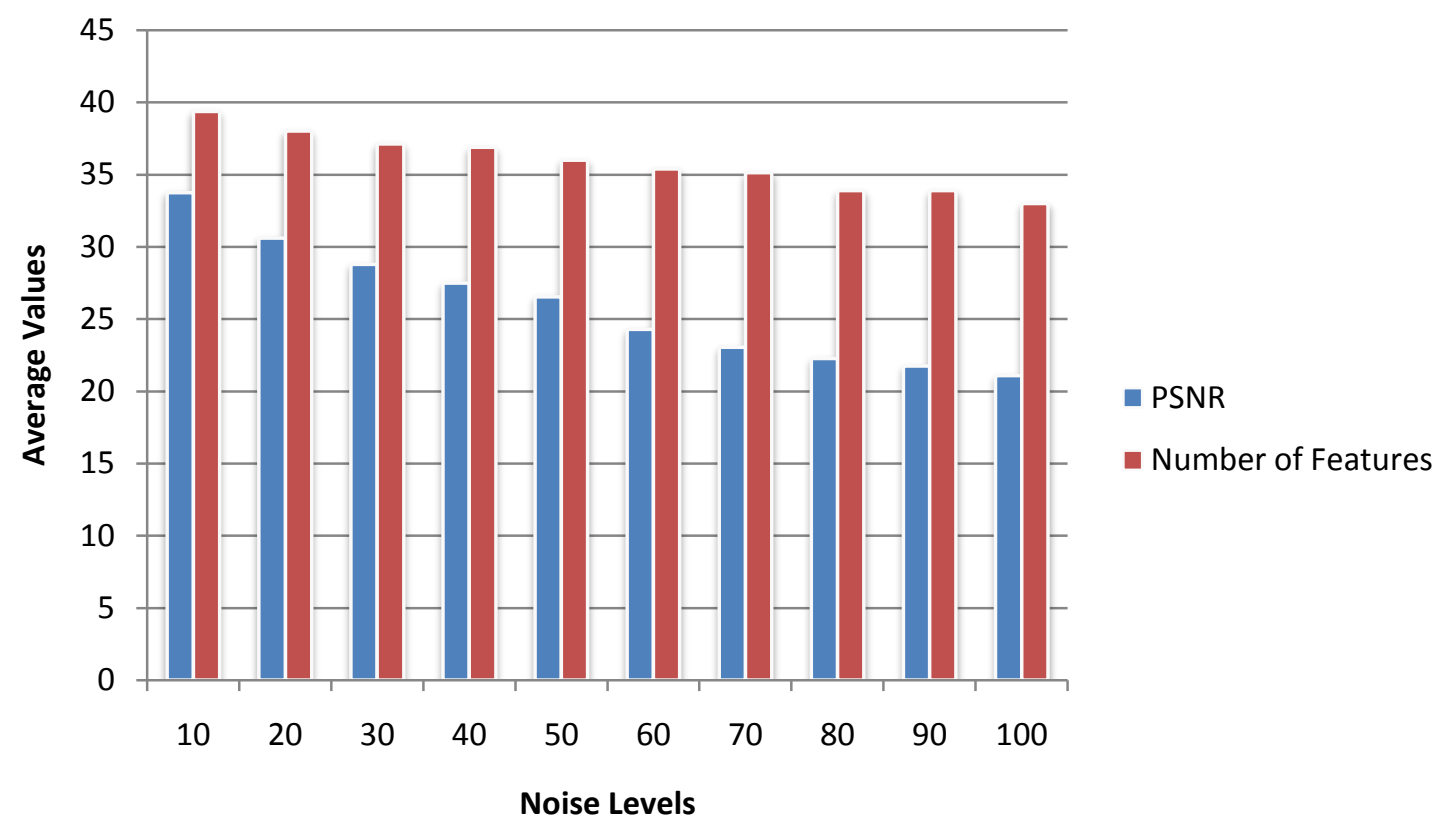

Figure 3 - 5: Average PSNR and average number of features comparison for patch size $7 \times 7$ and threshold value 5 over different noise levels. 


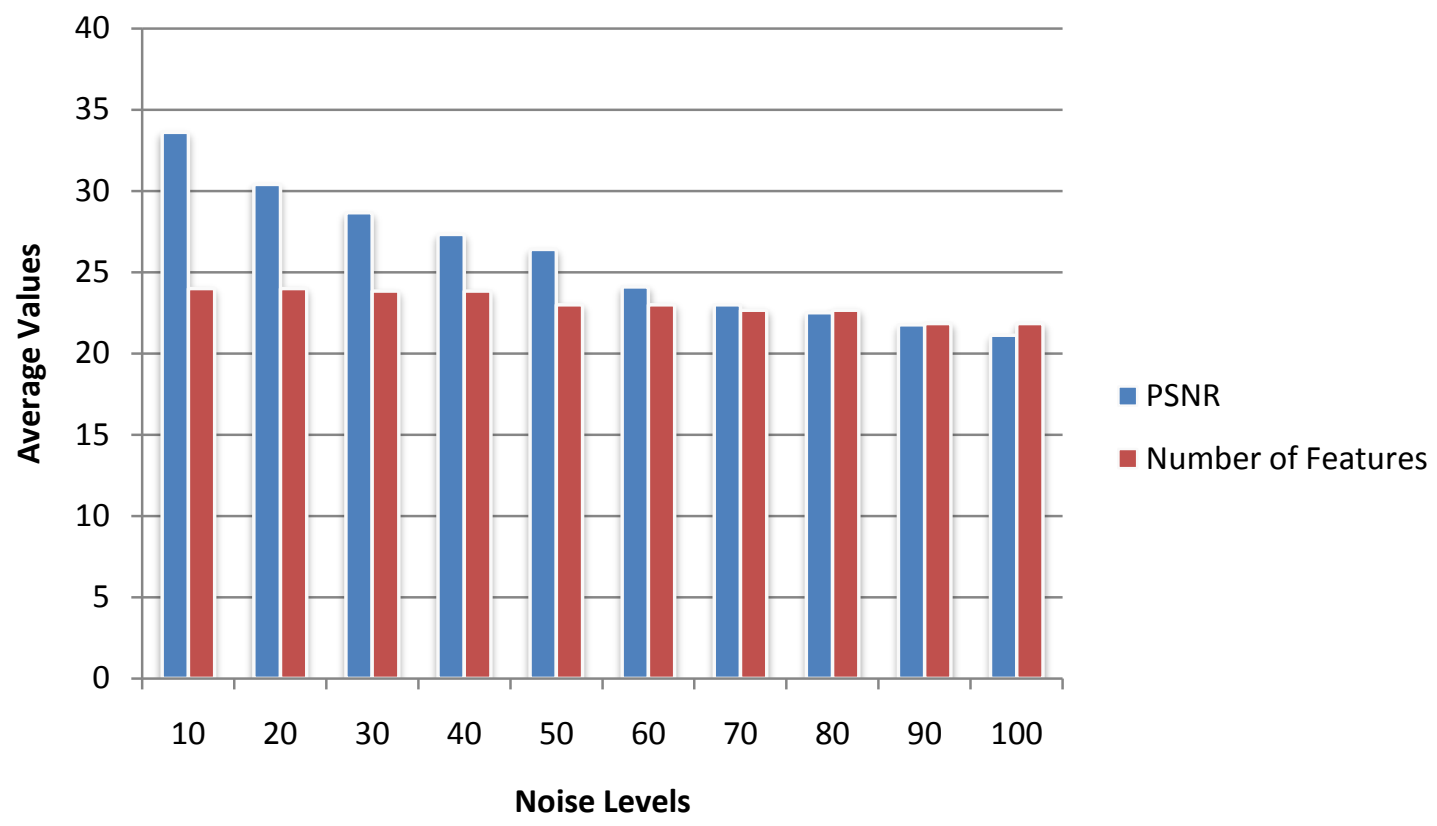

Figure 3 - 6: Average PSNR and average number of features comparison for patch size $5 \times 5$ and threshold value 3 over different noise levels.

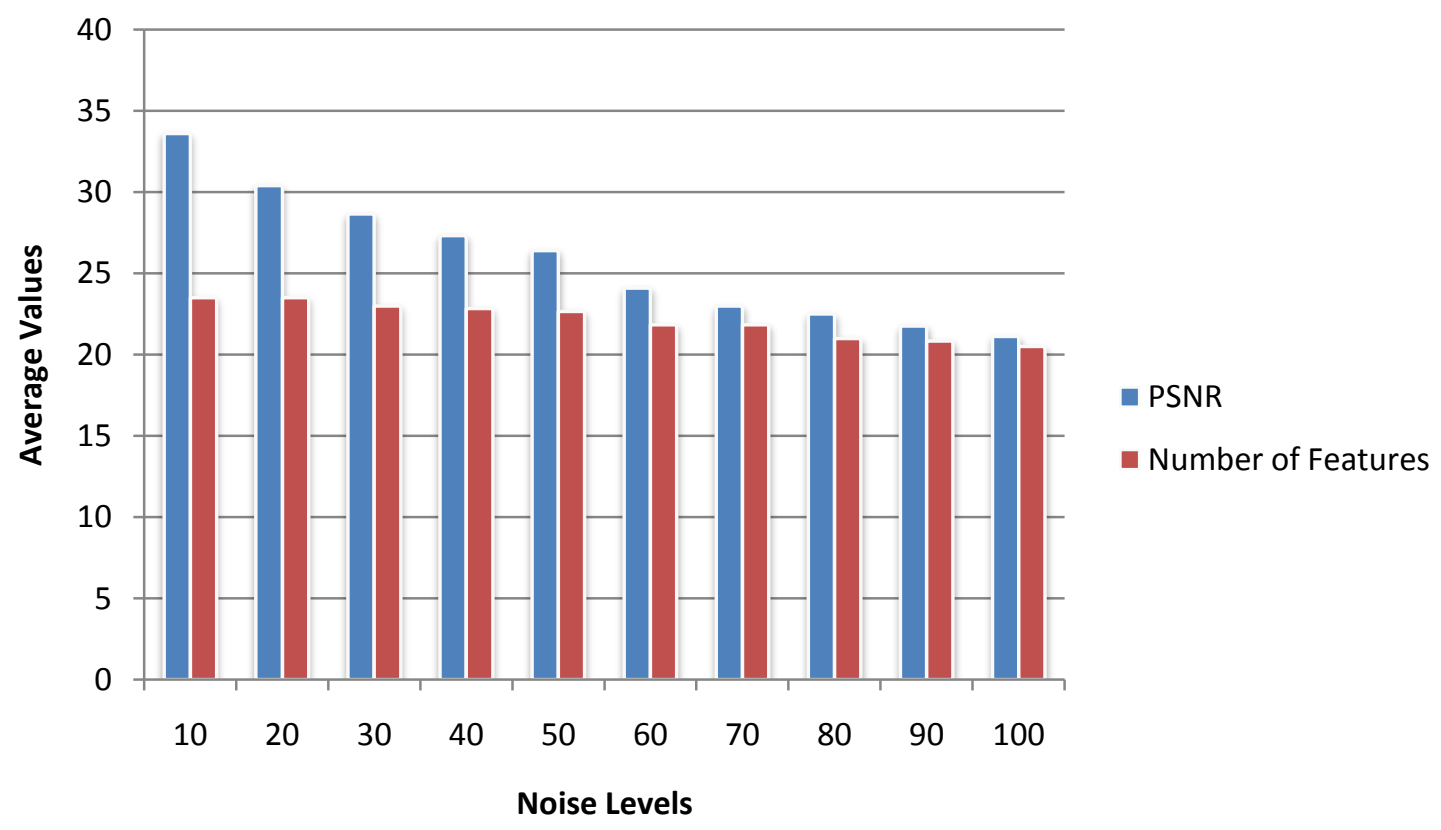

Figure 3 - 7: Average PSNR and average number of features comparison for patch size $5 \times 5$ and threshold value 5 over different noise levels. 


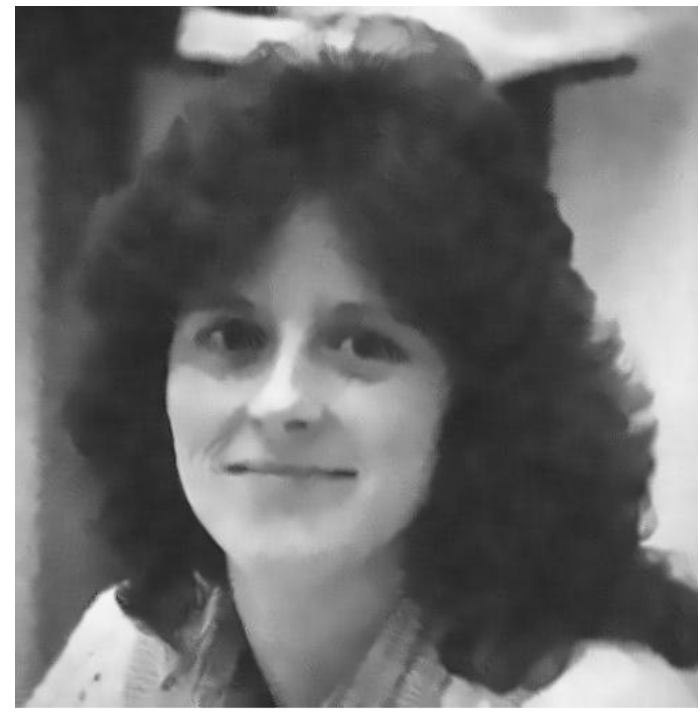

(a)

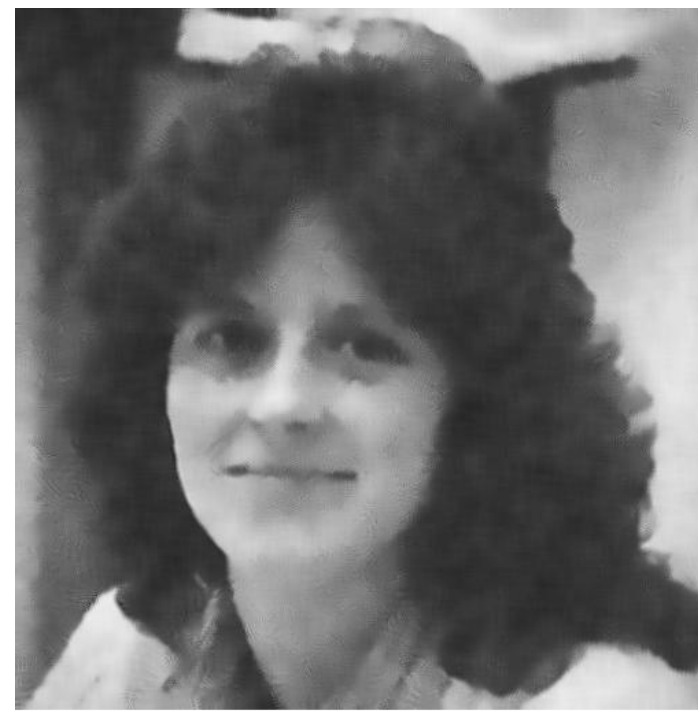

(c)

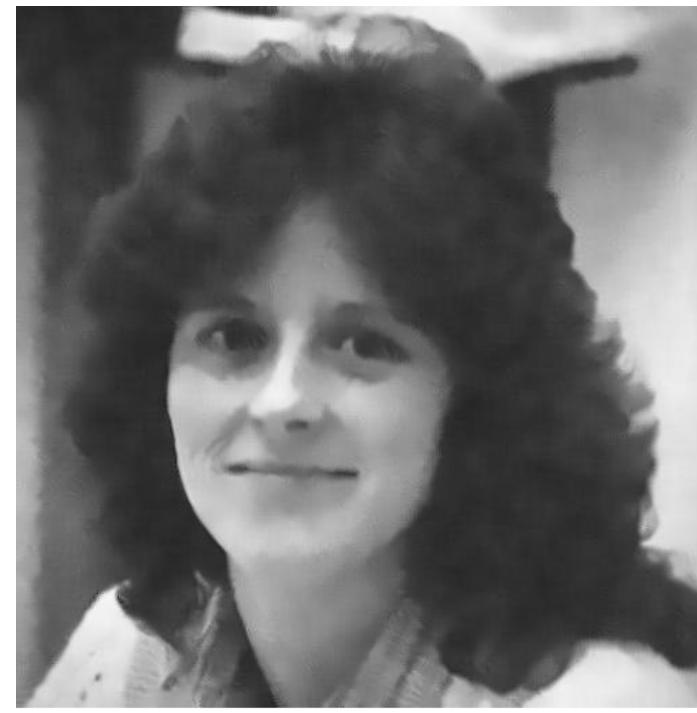

(b)

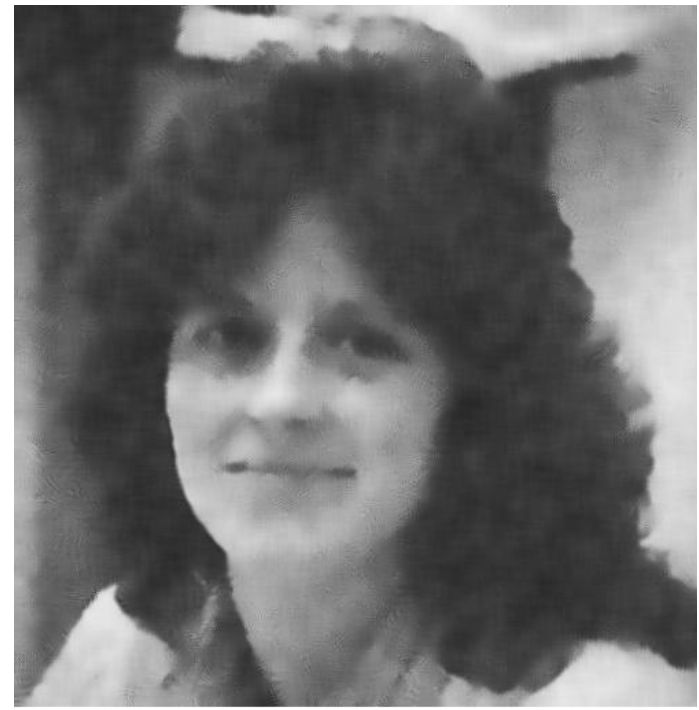

(d)

Figure 3 - 8: Performance analysis using Woman 1 image over different threshold values where noise level is $\sigma=40$ and patch size is $7 \times 7$ (a) threshold value $3, \operatorname{PSNR}=29.79$, (b) threshold value 5, PSNR $=29.79$, (c) threshold value 7, PSNR $=29.33$, and (d) threshold value $9, \mathrm{PSNR}=28.60$ 


\subsubsection{Selected Parameters}

To confirm the performance of our selected parameters, we have evaluated the effect of patch size by keeping the value of search region size and the threshold value constant.

Table 3-5 shows the effect of patch sizes while the search region size is $21 \times 21$ and the threshold value is 5 . It has been found that the patch size $7 \times 7$ performs the best. Table 3 6 shows the effect of patch size while the search region size is $15 \times 15$ and the threshold value is 5 . It has been found that patch size $5 \times 5$ performs the best. The bolded PSNR value represents the best results among these two tables. It has been found that for noise level $\sigma<80$, patch size $7 \times 7$ performs the best and for noise level $\sigma>80$, patch size $5 \times 5$ performs the best among these two tables.

Noise level estimation can be performed before assigning these parameters. Preclassification of homogeneous areas [28] [29], image filtering [30] [31], Wavelet transform [32] and local variance estimate [33] are most widely used models to estimate the noise from an image.

Finally, we can conclude that for noise level $\sigma<80$, patch size $7 \times 7$ performs the best. Whereas for noise level $\sigma>80$, patch size $5 \times 5$ performs the best (see Section 3.1.4.1). For patch size $7 \times 7$, search region size $21 \times 21$ performs the best and for patch size $5 \times 5$, search

region size $15 \times 15$ performs the best (see Section 3.1.4.2). Threshold value 5 shows the best result for these selected parameters (see Section 3.1.4.3). 
Table 3- 5: Average PSNR comparison for all test images between different patch sizes for different noise levels.

\begin{tabular}{|c|c|c|c|c|c|}
\hline Noise Level & $3 \times 3$ & $5 \times 5$ & $7 \times 7$ & $9 \times 9$ & $11 \times 11$ \\
\hline \multicolumn{6}{|c|}{ Search region size $21 \times 21$, threshold value $=5$} \\
\hline 10 & 32.09 & 33.62 & 33.75 & 32.91 & 32.06 \\
\hline 20 & 29.21 & 30.43 & 30.60 & 29.88 & 29.07 \\
\hline 30 & 27.52 & 28.64 & 28.78 & 28.07 & 27.28 \\
\hline 40 & 26.27 & 27.31 & 27.49 & 26.81 & 25.90 \\
\hline 50 & 25.41 & 26.41 & 26.54 & 25.92 & 25.03 \\
\hline 60 & 23.18 & 24.11 & 24.27 & 23.67 & 23.10 \\
\hline 70 & 21.93 & 22.99 & 23.03 & 22.45 & 22.08 \\
\hline 80 & 21.49 & 22.13 & 22.48 & 21.97 & 21.52 \\
\hline 90 & 20.78 & 21.38 & 21.65 & 21.13 & 20.85 \\
\hline 100 & 20.14 & 20.75 & 21.02 & 20.62 & 20.28 \\
\hline
\end{tabular}


Table 3- 6: Average PSNR comparison for all test images between different patch sizes for different noise levels.

\begin{tabular}{|c|c|c|c|c|c|}
\hline Noise Level & $3 \times 3$ & $5 \times 5$ & $7 \times 7$ & $9 \times 9$ & $11 \times 11$ \\
\hline \multicolumn{6}{|c|}{ Search region size $15 \times 15$, threshold value $=5$} \\
\hline 10 & 32.07 & 33.67 & 33.43 & 32.42 & 32.09 \\
\hline 20 & 29.21 & 30.39 & 30.31 & 29.41 & 29.07 \\
\hline 30 & 27.52 & 28.64 & 28.50 & 27.70 & 27.28 \\
\hline 40 & 26.30 & 27.32 & 27.23 & 26.46 & 25.91 \\
\hline 50 & 25.42 & 26.40 & 26.28 & 25.58 & 25.04 \\
\hline 60 & 23.18 & 24.10 & 24.03 & 23.35 & 23.10 \\
\hline 70 & 21.93 & 22.98 & 22.80 & 22.13 & 22.08 \\
\hline 80 & 21.41 & 22.52 & 22.23 & 22.09 & 21.47 \\
\hline 90 & 20.71 & 21.76 & 21.41 & 21.27 & 21.11 \\
\hline 100 & 20.01 & 21.12 & 20.89 & 20.71 & 20.08 \\
\hline
\end{tabular}




\section{Chapter 4}

\section{Experimental Results and Analysis}

We have tested our image denoising algorithm on a standard image set with noise standard deviation $\sigma$ ranging from 10 to 100 for additive white gaussian noise. We have compared our proposed method with the Non-Local Means algorithm as well as its variants. We have compared our results and analyzed it based on the PSNR and the SSIM measures. We also analyzed and compared the results subjectively.

\subsection{Data set}

We have used standard images for our test purpose. Figure 4-1 shows the test images. These standard images are initially noise free. We contaminated them with additive white gaussian noise for testing purpose.

\subsection{Noise Generation}

Noise can be defined as a deviation from the ideal signal. In general, additive noise is evenly distributed over the original image. To generate a noisy image for our testing purpose, the generated noise is added to the original image. The final signal is kept under the maximum range of intensity value of gray image. Figure 4-2 shows the example of a noisy image. 


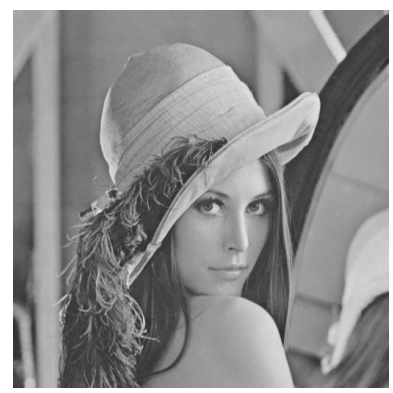

(a) Lena

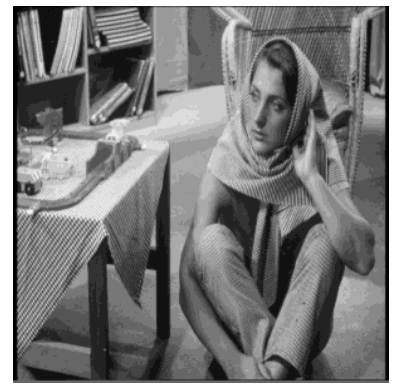

(e) Barbara

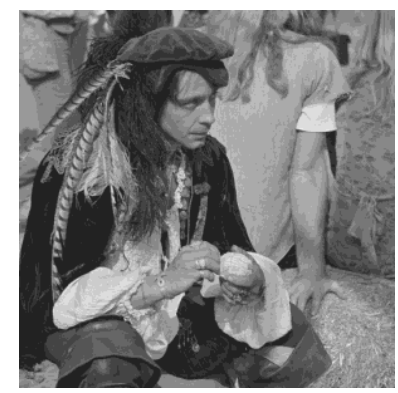

(b) Man

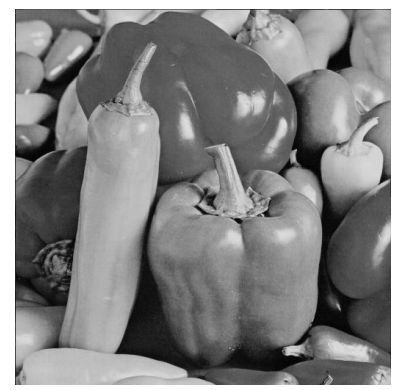

(f) Peppers

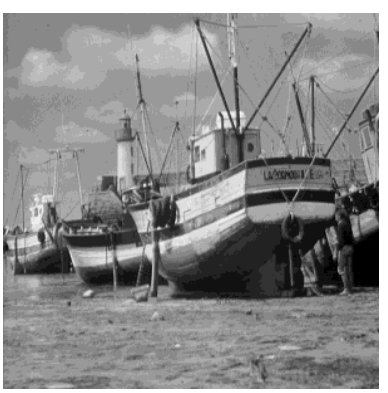

(c) Boat

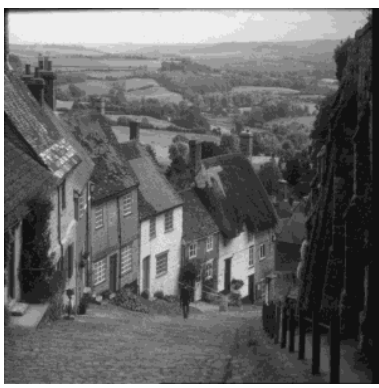

(g) Hill

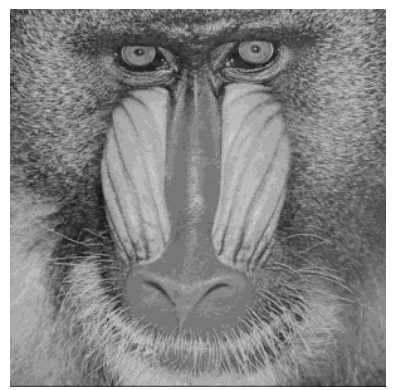

(d) Baboon

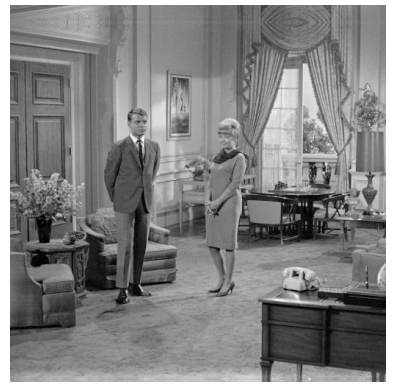

(h) Couple

Figure 4- 1: Set of images for performance analysis.

\subsection{Performance measure}

To evaluate the performance of our denoising algorithm we have used the Peak Signal to Noise Ratio (PSNR) and the Structural Similarity (SSIM) measure. These are widely used objective measures for evaluating the performance of image denoising algorithms. 


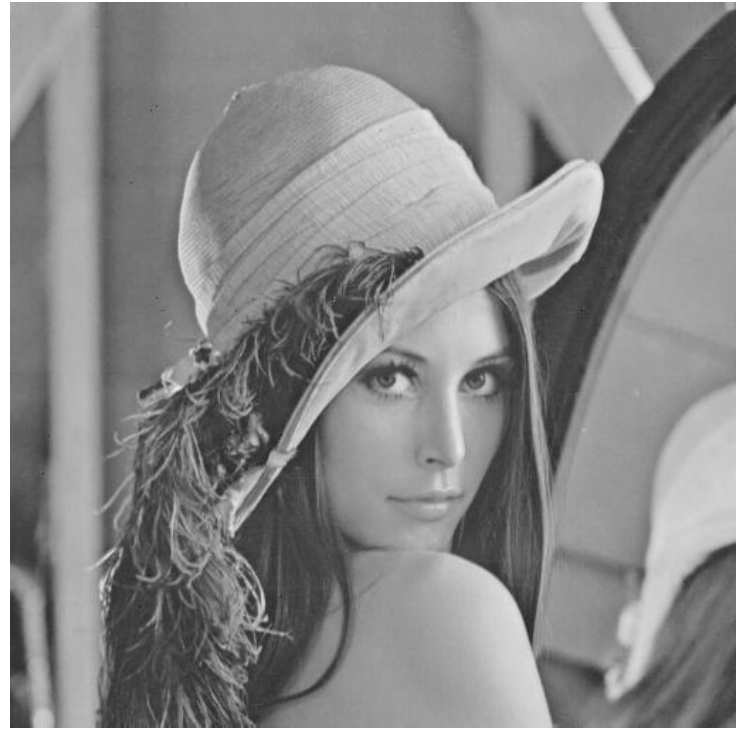

(a)

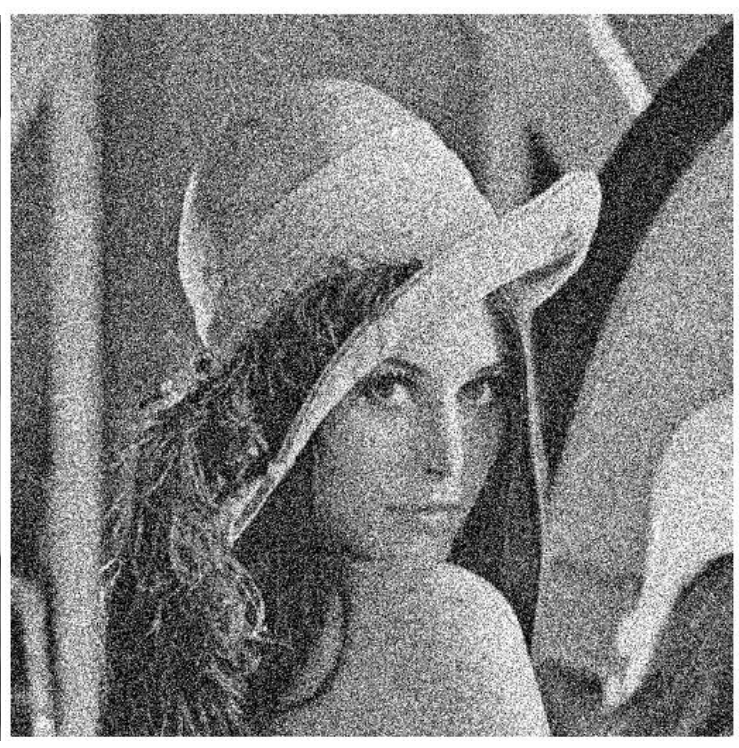

(b)

Figure 4- 2: Noise generation (a) Noise free image Lena. (b) Noisy image with Additive white Gaussian noise (noise level $\sigma=50$ ).

\subsubsection{Peak Signal to Noise Ratio}

The peak signal to noise ratio (PSNR) represents the ratio between the maximum powers of a signal to the noise which degrades the original image. This measure is based on the Mean Squared Error (MSE) which assesses the difference between the original image data and the degraded image data. Given the original image data $u_{i j}$ and the degraded image data $v_{i j}$ of size $M \times N$, MSE is defined as,

$$
\begin{gathered}
M S E=\frac{1}{M \times N} \sum_{i=0}^{M} \sum_{j=0}^{N}\left(u_{i j}-v_{i j}\right)^{2} \\
P S N R=10 \log _{10}\left\{\frac{M A X^{2}}{M S E}\right\}
\end{gathered}
$$


where MAX is the maximum possible pixel intensity value. High PSNR value indicates a better reconstruction or denoising. The drawback of PSNR is that it relies only on pixel numerical values rather than any structural similarity.

\subsubsection{Structural Similarity Index (SSIM)}

The structural similarity index is used to find similarity between two images. Similar pixels have strong inter-dependencies when they are closer. The following equation measures SSIM

$$
S S I M=\frac{\left(2 \mu_{x} \mu_{y}+c_{1}\right)\left(2 \sigma_{x y}+c_{2}\right)}{\left(\mu_{x}^{2}+\mu_{y}^{2}+c_{1}\right)\left(\sigma_{x}^{2}+\sigma_{y}^{2}+c_{2}\right)}
$$

Where, $x$ and $y$ are two windows of identical size. In this equation $\mu_{x}$ and $\mu_{y}$ are the average of $x$ and $y, \sigma_{x}^{2}$ and $\sigma_{y}^{2}$ are the variance of $x$ and $y$ and $\sigma_{x y}$ is the co-variance. $c_{1}=\left(k_{1} L\right)^{2}$ and $c_{2}=\left(k_{2} L\right)^{2} ; k_{1} \ll 1, k_{2} \ll 1$ and $L$ is the dynamic range of pixel values.

\subsection{Results and Analysis}

\subsubsection{Parameter Setting}

The performance of the proposed method is assessed using peak signal to noise ratio (PSNR) and the structural similarity measure (SSIM). For noise levels $\sigma<80$, we have considered the patch size to be $7 \times 7$, the search region size to be $21 \times 21$ and the threshold value to be 5 . For noise levels $\sigma>80$, the patch size is set to be $5 \times 5$, the search region size is set to be $15 \times 15$ and the threshold value is set to be 5 (see Section 3.1.5). During the rest of our results and analysis we have used these parameters for our proposed method. We have used Matlab R2013b (version 8.2.0.29) for our implementation purposes. All of the results are produced using $2.30 \mathrm{GHz}$ Intel(R) Core i5 processor with 4GB RAM 
under Linux OS using Ubuntu 14.1. The execution time was recorded in milliseconds. All of the test results are recorded and averaged after 10 runs.

\subsubsection{Performance analysis using PSNR}

The performance of our proposed method is compared in terms of PSNR with other denoising schemes, namely the original NLM method, the principal component analysis based NLM method (PCA-NLM), the patch regression based NLM method (NLMPatch) and the BM3D method.

Table 4-1 and Table 4-2 show the comparative performance for Lena image and the average comparative performance for all test images at different noise levels, respectively. The bolded values represent the highest PSNR value among all of the algorithms for a given noise level. Figure 4-3 compares the average PSNR for the proposed method and all other denoising algorithms.

It has been found that, the proposed method performs better than all other methods except BM3D. In case of the BM3D method, the proposed method performs better than the BM3D method only when $\sigma<50$. The BM3D method performs better at the higher noise levels. 
Table 4-1: PSNR $(\mathrm{dB})$ comparison for Lena image among the proposed method, the NLM method, variants of the NLM method and the BM3D method for different noise levels.

\begin{tabular}{|c|c|c|c|c|c|}
\hline Noise Level & NLM & PCA-NLM & NLM-Patch & $\begin{array}{c}\text { Proposed } \\
\text { Method } \\
\end{array}$ & BM3D \\
\hline 10 & 34.57 & 34.58 & 33.21 & 35.91 & 35.79 \\
\hline 20 & 31.89 & 31.92 & 30.09 & 33.32 & 32.94 \\
\hline 30 & 30.0 & 31.05 & 27.71 & 31.55 & 31.16 \\
\hline 40 & 28.42 & 29.02 & 25.85 & 29.97 & 29.79 \\
\hline 50 & 27.10 & 26.99 & 25.46 & 27.65 & 28.70 \\
\hline 60 & 25.55 & 24.98 & 23.89 & 25.98 & 28.27 \\
\hline 70 & 23.99 & 24.01 & 23.10 & 24.93 & 27.57 \\
\hline 80 & 23.05 & 23.51 & 22.82 & 23.96 & 26.97 \\
\hline 90 & 22.99 & 22.98 & 21.95 & 23.09 & 26.45 \\
\hline 100 & 22.18 & 22.19 & 21.17 & 22.29 & 25.95 \\
\hline Average & 26.97 & 27.12 & 25.52 & 27.86 & 29.36 \\
\hline
\end{tabular}


Table 4-2: Average PSNR(dB) comparison for all test images among the proposed method, the NLM method, variants of the NLM method and the BM3D method for different noise levels.

\begin{tabular}{|c|c|c|c|c|c|}
\hline Noise Level & NLM & PCA-NLM & NLM-Patch & $\begin{array}{c}\text { Proposed } \\
\text { Method }\end{array}$ & BM3D \\
\hline 10 & 32.52 & 32.94 & 31.47 & 33.94 & 33.84 \\
\hline 20 & 29.87 & 29.95 & 29.04 & 31.0 & 30.50 \\
\hline 30 & 28.13 & 28.26 & 27.45 & 28.96 & 28.38 \\
\hline 40 & 26.69 & 26.43 & 25.87 & 27.72 & 27.70 \\
\hline 50 & 25.49 & 25.38 & 24.61 & 26.49 & 26.86 \\
\hline 60 & 23.85 & 23.87 & 22.75 & 24.30 & 25.94 \\
\hline 70 & 22.90 & 22.81 & 22.31 & 23.22 & 25.29 \\
\hline 80 & 22.32 & 22.32 & 21.92 & 22.60 & 24.75 \\
\hline 90 & 21.73 & 21.57 & 20.89 & 21.86 & 24.18 \\
\hline 100 & 21.13 & 20.94 & 20.14 & 21.19 & 23.68 \\
\hline Average & 25.46 & 25.45 & 24.64 & 26.15 & 27.11 \\
\hline
\end{tabular}




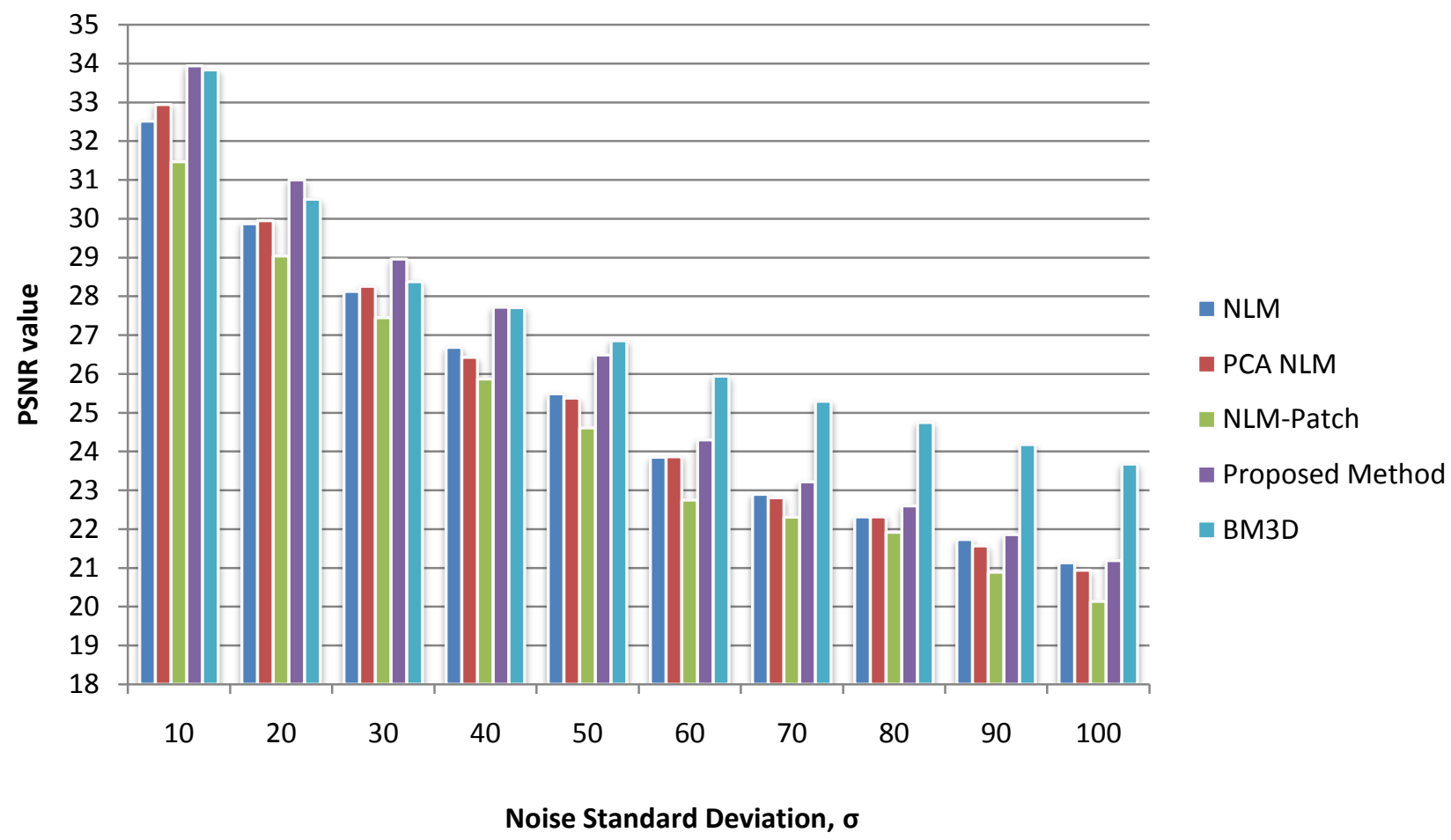

Figure 4- 3: Bar graph for average PSNR comparison for the proposed method, the NLM method, variants of the NLM method and the BM3D method for different noise levels.

\subsubsection{Performance analysis using SSIM}

Table 4-3 and Table 4-4 show the SSIM comparison for Lena image and the average SSIM comparison for all test images between the proposed method and the other denoising schemes, respectively. The bold face digits represent the highest SSIM value among all of these algorithms. Figure 4-4 compares average SSIM between the proposed method and other the denoising schemes.

For noise level $\sigma<50$, the proposed method performs better than all other denoising schemes. Yet, for noise level $\sigma>50$ the proposed method performs better than the original NLM and its variants. The BM3D performs better at higher noise levels.

Appendix A exhibits further analysis on Pepper, Boat and Couple image. 
Table 4-3: SSIM comparison for Lena image among the proposed method, the NLM method, variants of the NLM method and the BM3D method for different noise levels.

\begin{tabular}{|c|c|c|c|c|c|}
\hline Noise Level & NLM & PCA-NLM & NLM-Patch & $\begin{array}{l}\text { Proposed } \\
\text { Method }\end{array}$ & BM3D \\
\hline 10 & 0.9107 & 0.9097 & 0.9047 & 0.9218 & 0.9155 \\
\hline 20 & 0.8712 & 0.8695 & 0.8654 & 0.8917 & 0.8749 \\
\hline 30 & 0.8401 & 0.8391 & 0.8372 & 0.8508 & 0.8410 \\
\hline 40 & 0.8094 & 0.8071 & 0.8042 & 0.8113 & 0.8083 \\
\hline 50 & 0.7589 & 0.7597 & 0.7581 & 0.7714 & 0.7799 \\
\hline 60 & 0.7412 & 0.7387 & 0.7381 & 0.7512 & 0.7567 \\
\hline 70 & 0.7151 & 0.7147 & 0.7128 & 0.7324 & 0.7359 \\
\hline 80 & 0.7096 & 0.7072 & 0.7052 & 0.7216 & 0.7344 \\
\hline 90 & 0.7017 & 0.6915 & 0.7055 & 0.7109 & 0.7259 \\
\hline 100 & 0.6714 & 0.6621 & 0.6617 & 0.7008 & 0.7134 \\
\hline Average & 0.7928 & 0.7883 & 0.7876 & 0.8064 & 0.8073 \\
\hline
\end{tabular}


Table 4-4: Average SSIM comparison for all test images among the proposed method, the NLM method, variants of the NLM method and the BM3D method for different noise levels.

\begin{tabular}{|c|c|c|c|c|c|}
\hline Noise Level & NLM & PCA-NLM & NLM-Patch & $\begin{array}{c}\text { Proposed } \\
\text { Method } \\
\end{array}$ & BM3D \\
\hline 10 & 0.9078 & 0.9015 & 0.9051 & 0.9201 & 0.9124 \\
\hline 20 & 0.8625 & 0.8605 & 0.8610 & 0.8785 & 0.8711 \\
\hline 30 & 0.8389 & 0.8341 & 0.8291 & 0.8469 & 0.8415 \\
\hline 40 & 0.8071 & 0.8065 & 0.8017 & 0.8202 & 0.8201 \\
\hline 50 & 0.7689 & 0.7597 & 0.7659 & 0.7810 & 0.7841 \\
\hline 60 & 0.7487 & 0.7491 & 0.7412 & 0.7524 & 0.7617 \\
\hline 70 & 0.7059 & 0.7032 & 0.7015 & 0.7195 & 0.7217 \\
\hline 80 & 0.6925 & 0.6912 & 0.6907 & 0.7079 & 0.7138 \\
\hline 90 & 0.6857 & 0.6815 & 0.6851 & 0.6992 & 0.7051 \\
\hline 100 & 0.6711 & 0.6504 & 0.6522 & 0.6975 & 0.7004 \\
\hline Average & 0.7878 & 0.7819 & 0.7823 & 0.8022 & 0.8099 \\
\hline
\end{tabular}




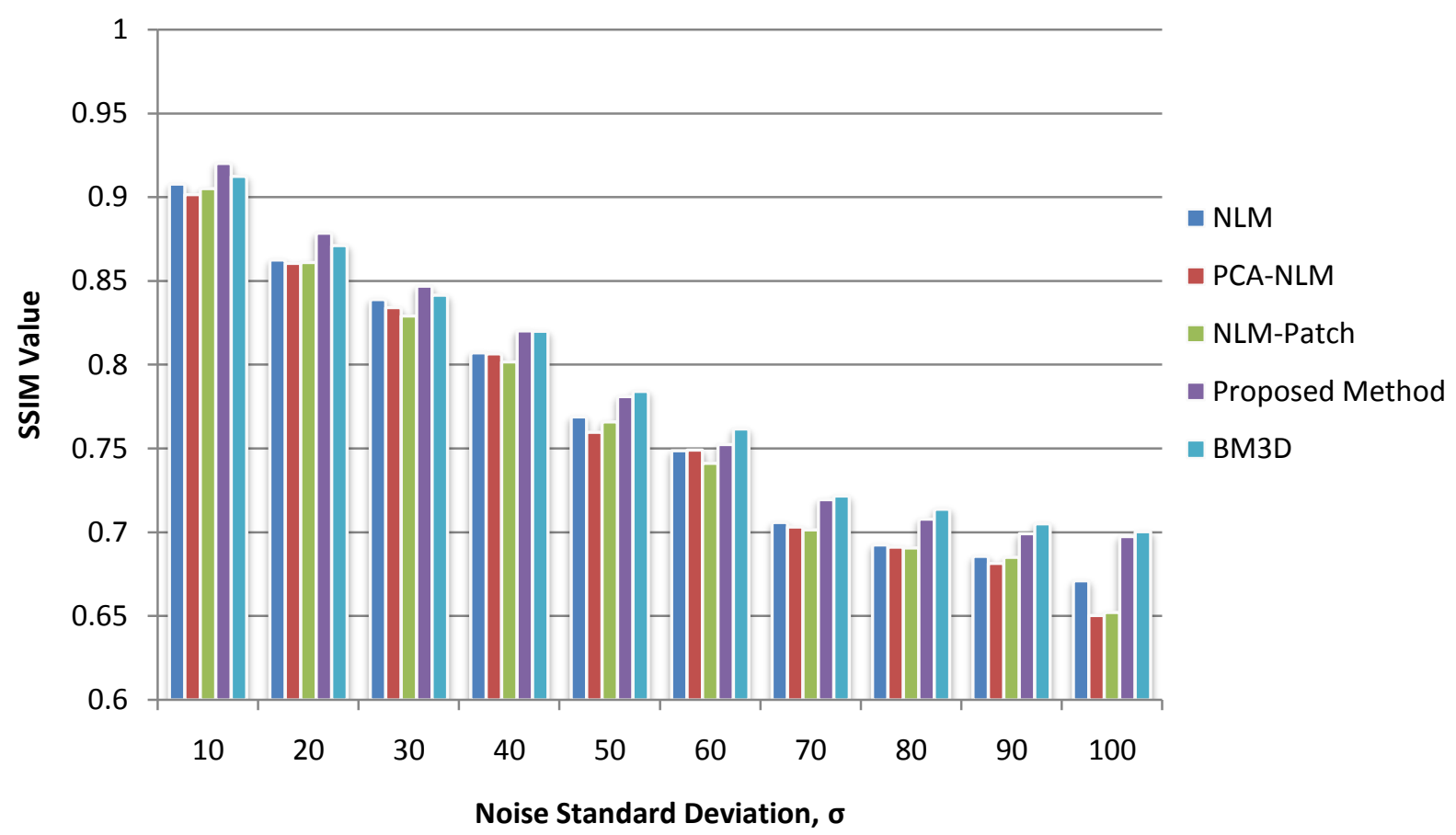

Figure 4- 4: Bar graph for average SSIM comparison for all test images among the proposed method, the NLM method, variants of the NLM method and the BM3D method for different noise levels.

\subsubsection{Running time performance analysis}

Table 4-5 and Table 4-6 compare the running time performance for Lena image and the average running time performance for all test images between the proposed method and the other denoising schemes, respectively. Figure 4-5 shows the average running time for the proposed method and all other denoising algorithms. It has been found that our proposed method outperforms the NLM method, variants of the NLM method and the BM3D method at all noise levels, as it requires fewer features to compare and calculate weights. Thus the computational time is dramatically reduced while keeping the denoising performance in an acceptable range. 
Table 4-5: Running time (in milliseconds) performance analysis for Lena image among the proposed method, the NLM method, variants of the NLM method and the BM3D method for different noise levels.

\begin{tabular}{|c|c|c|c|c|c|}
\hline Noise Level & NLM & PCA - NLM & NLM-Patch & $\begin{array}{c}\text { Proposed } \\
\text { Method }\end{array}$ & BM3D \\
\hline 10 & 209.9 & 195.5 & 208.2 & 151.8 & 224.8 \\
\hline 20 & 210.4 & 196.6 & 209.8 & 162.9 & 225.4 \\
\hline 30 & 211.1 & 198.1 & 209.9 & 163.1 & 228.9 \\
\hline 40 & 211.8 & 200.1 & 210.1 & 174.5 & 229.5 \\
\hline 50 & 212.7 & 201.2 & 211.5 & 181.9 & 230.8 \\
\hline 60 & 212.8 & 205.2 & 211.8 & 182.8 & 231.1 \\
\hline 70 & 214.0 & 208.7 & 212.4 & 183.0 & 232.5 \\
\hline 80 & 214.6 & 209.1 & 213.3 & 183.6 & 233.6 \\
\hline 90 & 216.9 & 209.7 & 214.7 & 184.2 & 234.6 \\
\hline 100 & 217.5 & 210.1 & 214.8 & 185.0 & 235.1 \\
\hline Average & 213.2 & 203.4 & 211.7 & 175.3 & 230.6 \\
\hline
\end{tabular}


Table 4-6: Average running time (in milliseconds) performance analysis for all test images among the proposed method, the NLM method, variants of the NLM method and the BM3D method for different noise levels.

\begin{tabular}{|c|c|c|c|c|c|}
\hline Noise Level & NLM & PCA-NLM & NLM-Patch & $\begin{array}{c}\text { Proposed } \\
\text { Method }\end{array}$ & BM3D \\
\hline 10 & 209.5 & 195.1 & 208.1 & 161.2 & 223.2 \\
\hline 20 & 210.7 & 196.7 & 210.6 & 164.5 & 224.2 \\
\hline 30 & 212.3 & 197.4 & 210.0 & 165.7 & 225.1 \\
\hline 40 & 212.6 & 198.8 & 211.5 & 169.9 & 229.3 \\
\hline 50 & 212.4 & 200.3 & 211.0 & 173.9 & 230.2 \\
\hline 60 & 213.0 & 204.4 & 212.8 & 181.2 & 230.8 \\
\hline 70 & 214.5 & 207.9 & 213.1 & 182.5 & 231.3 \\
\hline 80 & 214.9 & 208.6 & 213.9 & 184.0 & 231.6 \\
\hline 90 & 216.0 & 209.9 & 214.0 & 185.2 & 232.9 \\
\hline 100 & 217.1 & 210.1 & 216.4 & 185.9 & 233.1 \\
\hline Average & 213.3 & 202.9 & 212.1 & 175.4 & 229.8 \\
\hline
\end{tabular}




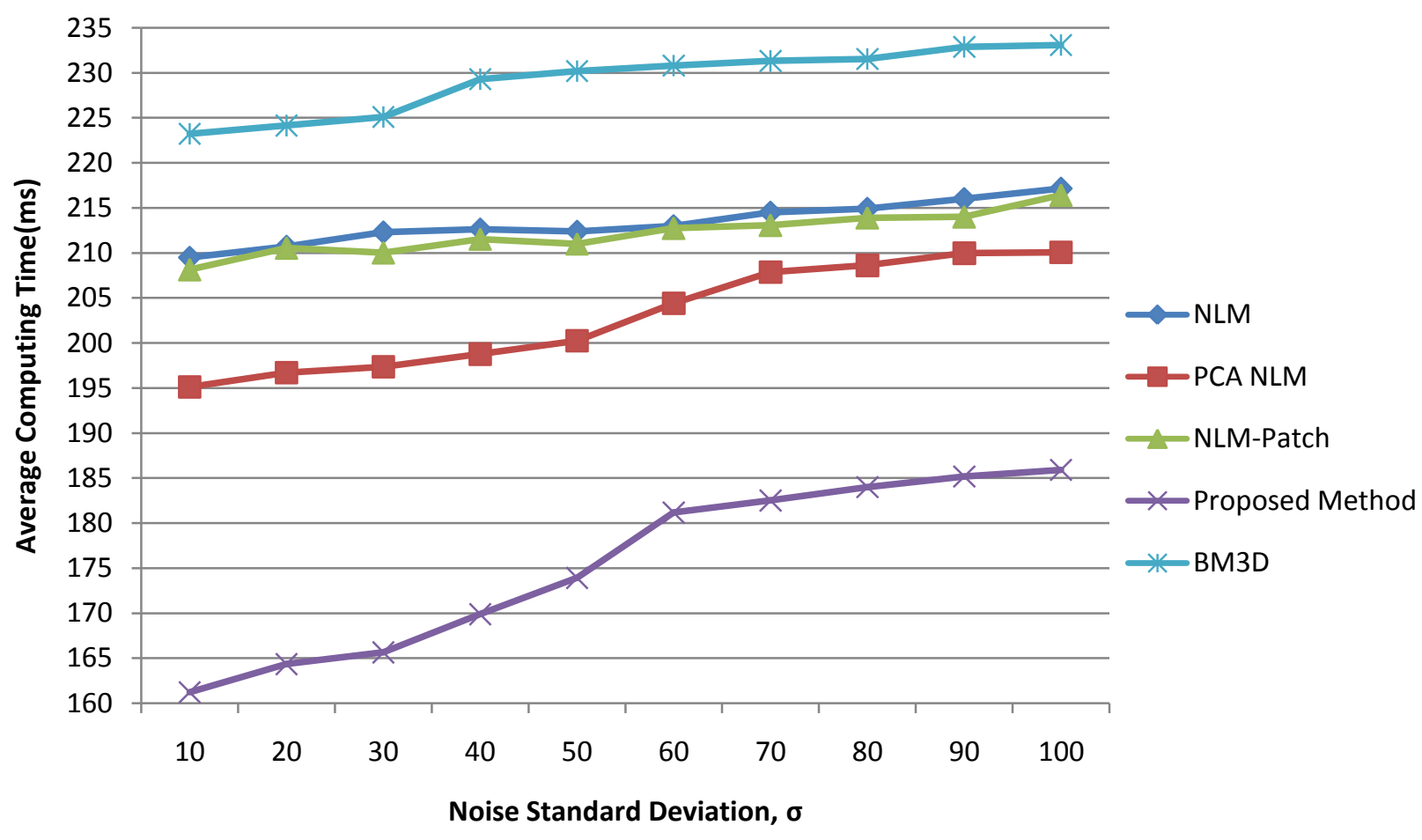

Figure 4- 5: Performance analysis for the average running time (in milliseconds) among the proposed method, the NLM method, variants of the NLM method and the BM3D method for different noise levels.

\subsubsection{Visual quality comparison and intensity profile}

\subsubsection{Visual quality comparison}

Producing visually improved image is an important preprocessing step in computer vision. Visually improved images can help many image processing algorithms to perform better. So, the visual quality comparison is one of the important criteria to measure the performance of the denoising algorithms. 
Figure 4-6 to Figure 4-10 show the output of the proposed method and the original NLM method on Lena image at different noise levels. We have also presented the same comparison on Peppers, Boat and Couple image in Appendix A. From Figure 4-6 (c) and Figure 4-6 (d) we can find that Figure 4-6 (d) is clear in the area of the feather if it is compared with Figure 4-6 (c). Also in the cap area for Lena image, Figure 4-6 (d) shows more clear stripes when it is compared with Figure 4-6 (c). Reported PSNR value also supports our claim that the proposed method is better than the original NLM method for noise level $\sigma=20$. Figure 4-7 to Figure 4-10 also supports that the proposed method performs better than the original NLM method at all noise levels.

Figure 4-11 compares the denoising performance on a zoomed area from Lena image. By observing the outputs, it has been found that the proposed method provides better performance over the original Non-Local Means in terms of edge preservation. By observing closely to the white bar area Figure 4-11 (d) and Figure 4-11(e), we can find that the proposed method could preserve the edges perfectly over the original Non-Local Means algorithm. 


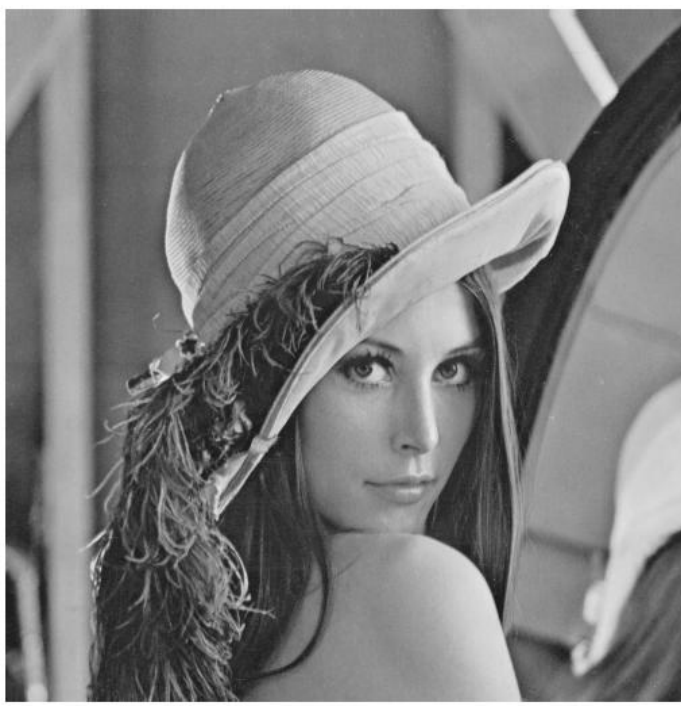

(a)

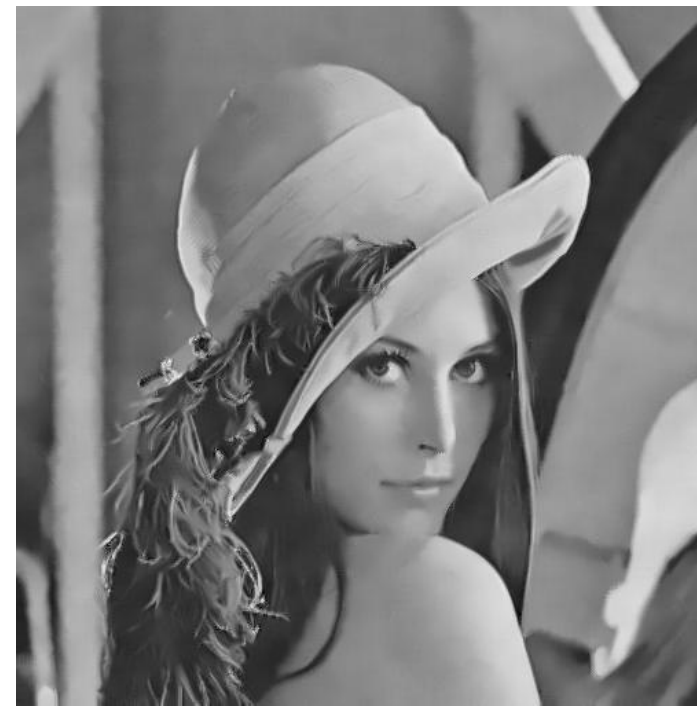

(c)

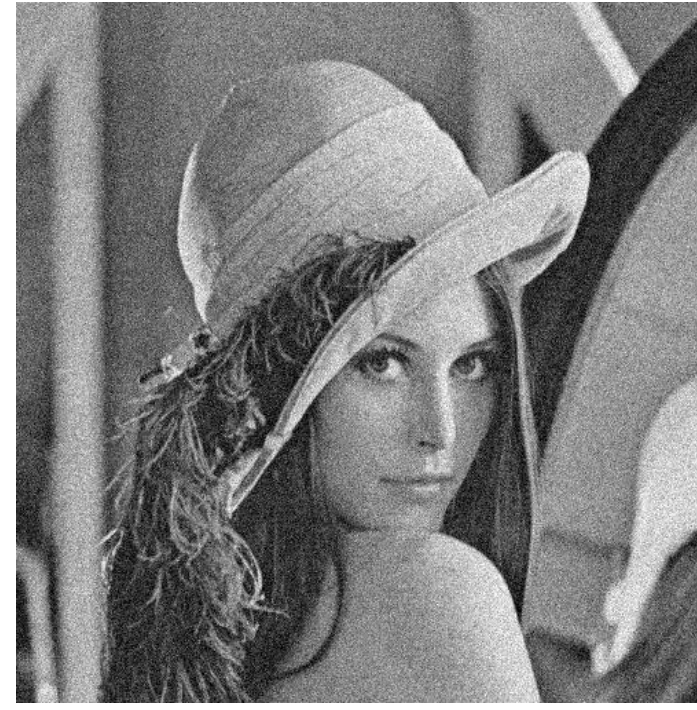

(b)

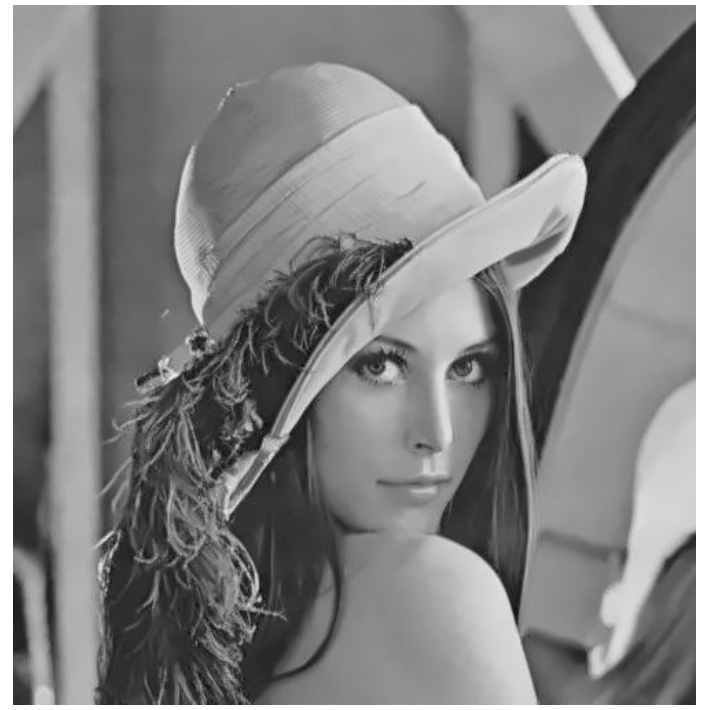

(d)

Figure 4- 6: Subjective comparison for denoising performance for Non-Local Means at noise level $\sigma=20$. (a) Noise free image Lena, (b) noisy image with Additive white Gaussian noise, (c) denoised image using the NLM method, PSNR=31.89, and (d) denoised image using the proposed method, PSNR $=33.32$. 


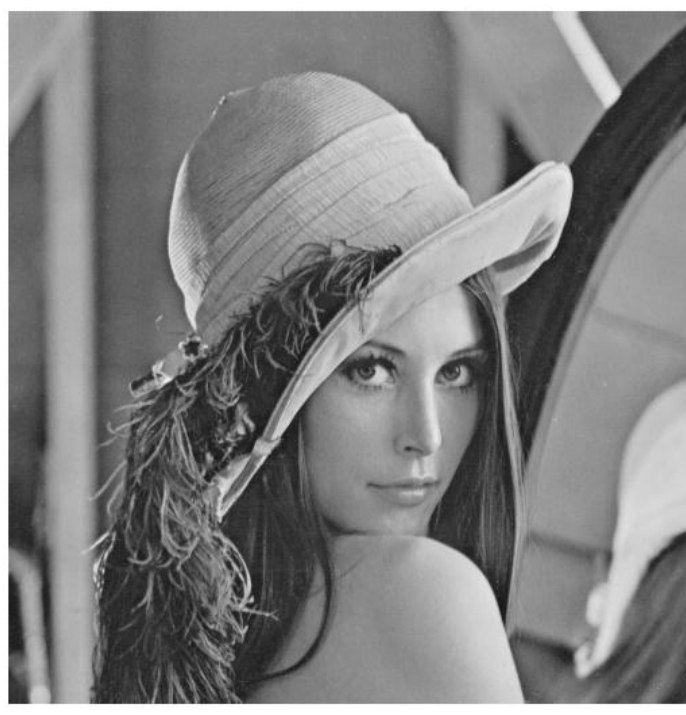

(a)

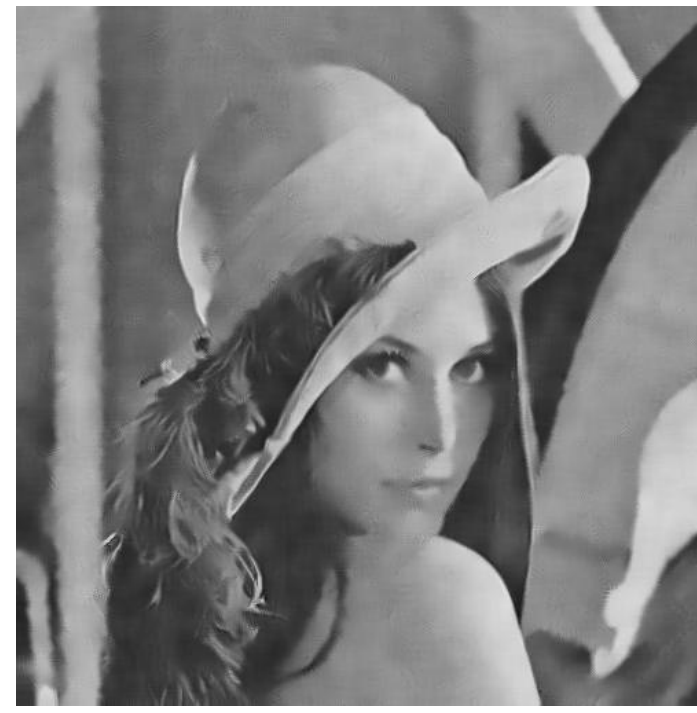

(c)

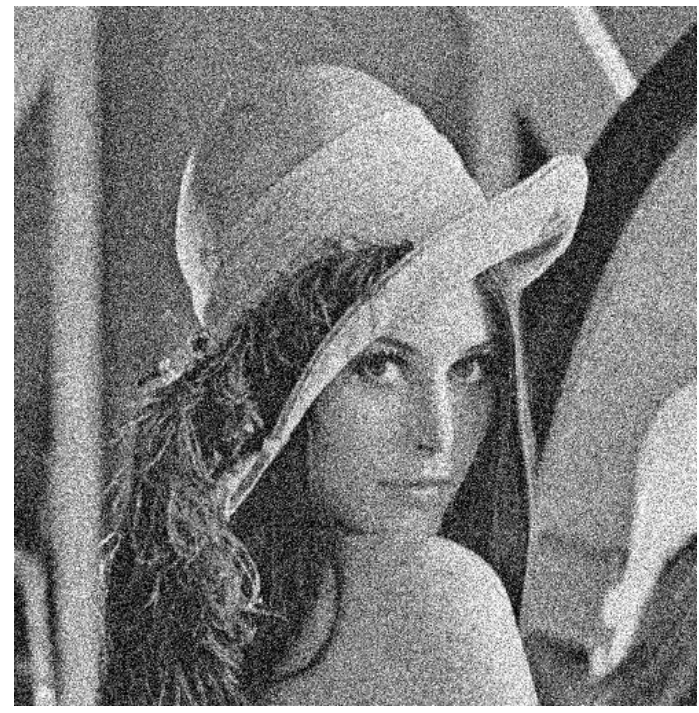

(b)

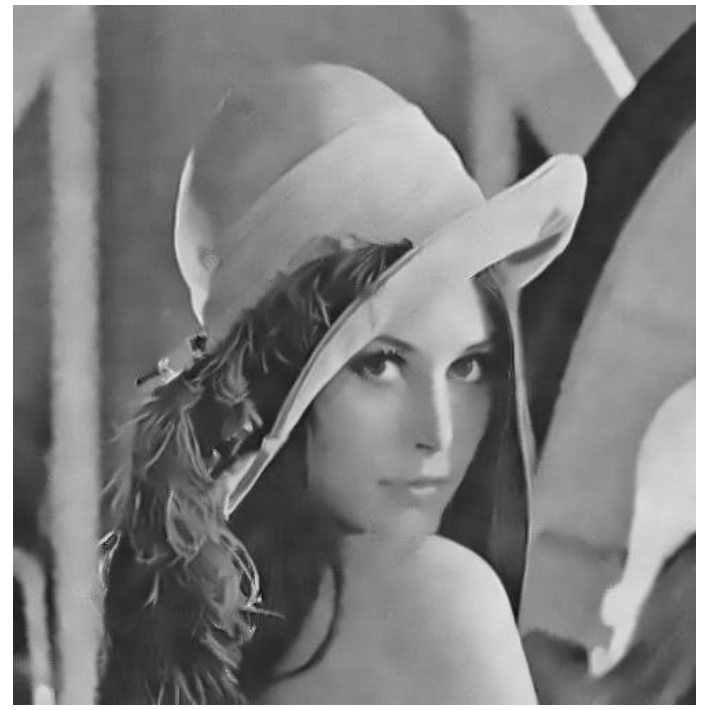

(d)

Figure 4- 7: Subjective comparison for denoising performance for Non-Local Means at noise level $\sigma=40$. (a) Noise free image Lena, (b) noisy image with Additive white Gaussian noise, (c) denoised image using the NLM method, PSNR= 28.42, and (d) denoised image using the proposed method, PSNR $=29.97$. 


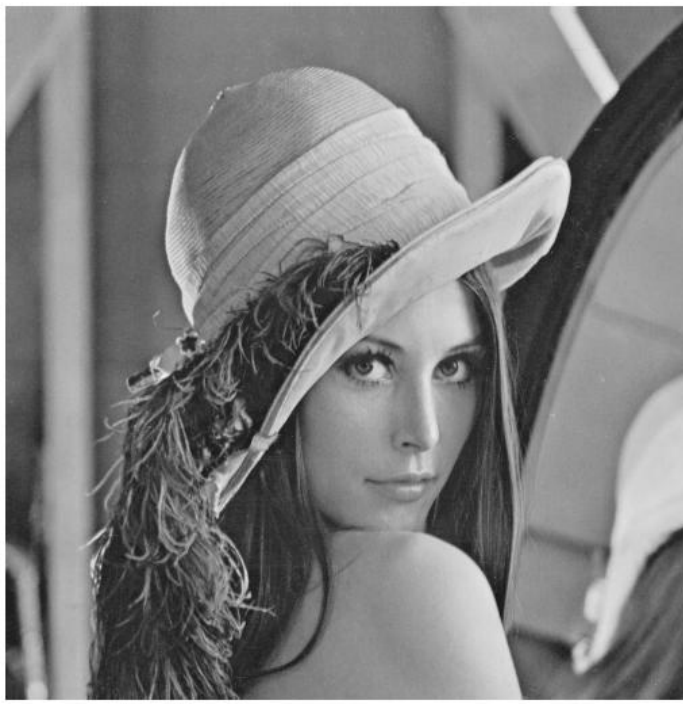

(a)

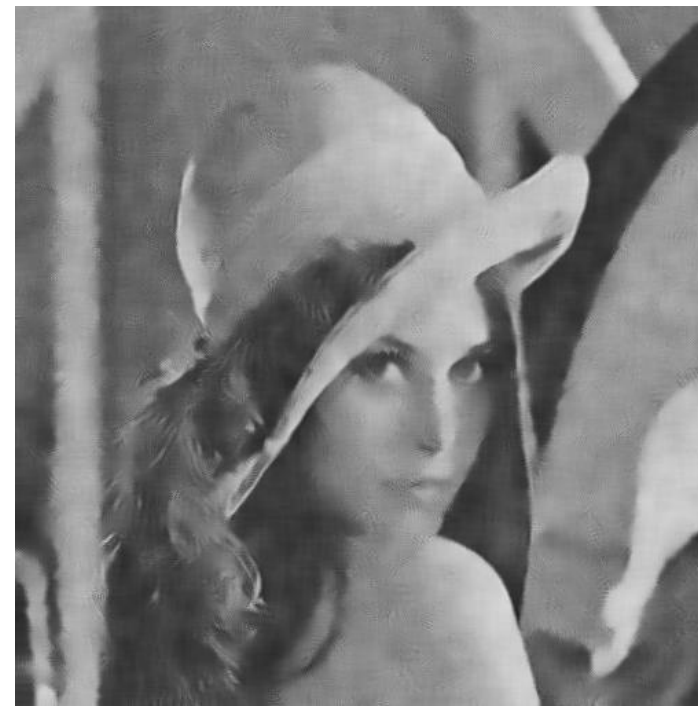

(c)

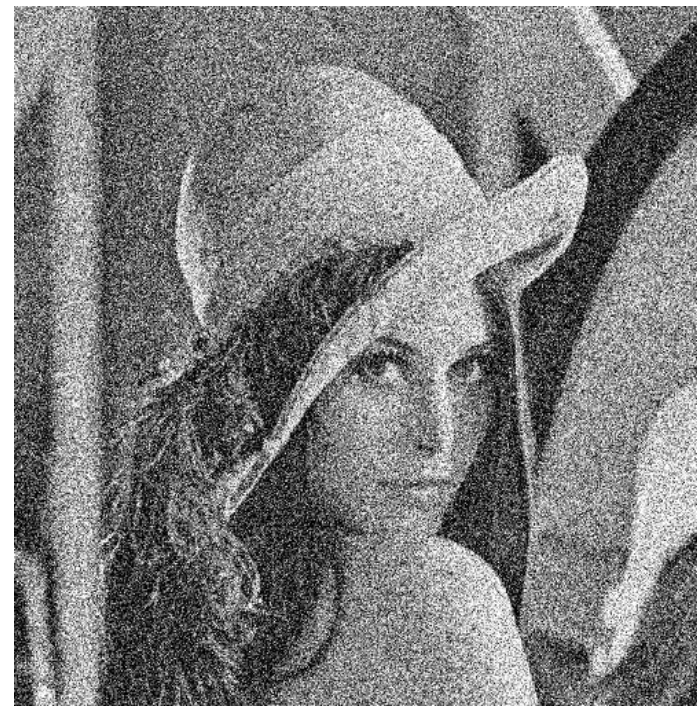

(b)

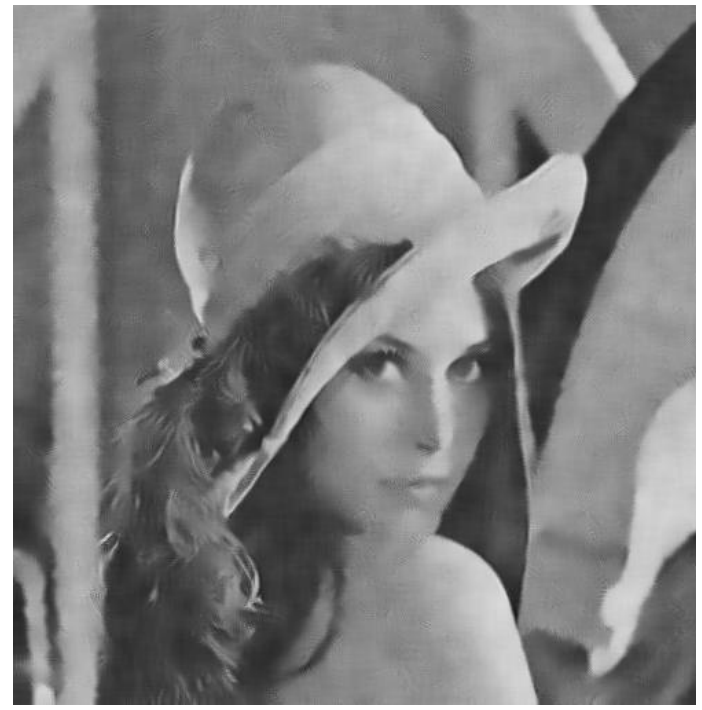

(d)

Figure 4- 8: Subjective comparison for denoising performance for Non-Local Means at noise level $\sigma=60$. (a) Noise free image Lena, (b) noisy image with Additive white Gaussian noise, (c) denoised image using the NLM method, PSNR= 25.55, and (d) denoised image using the proposed method, PSNR $=25.98$. 


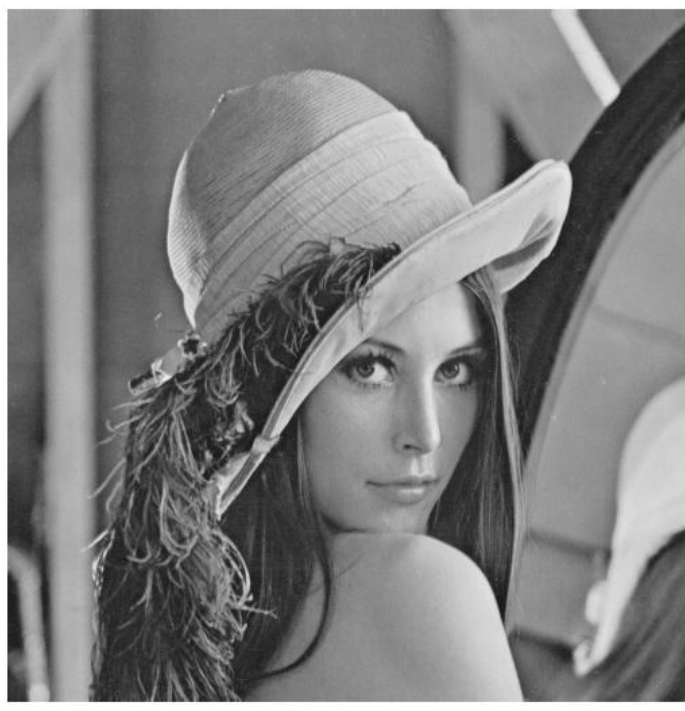

(a)

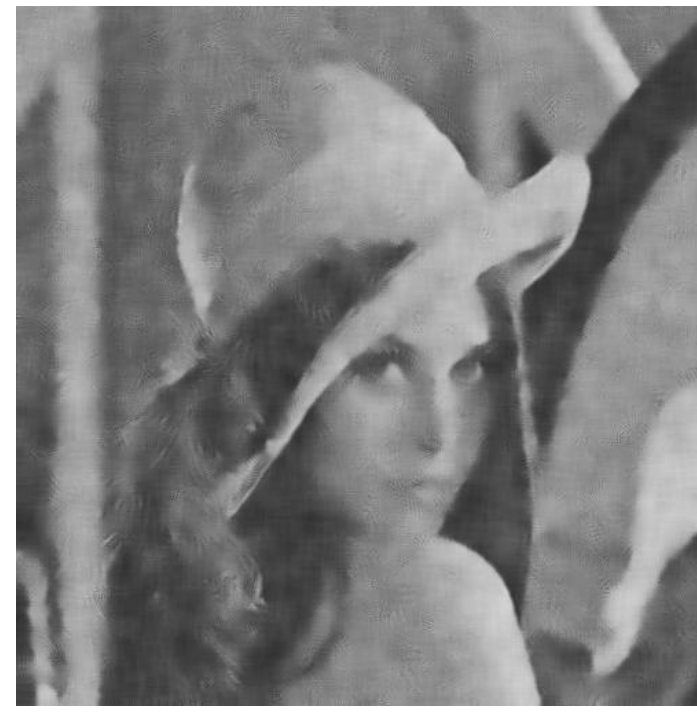

(c)

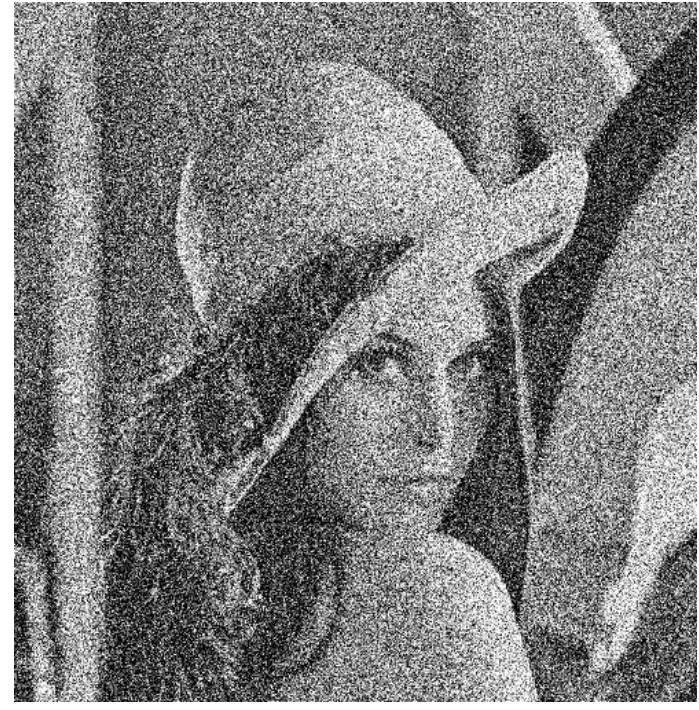

(b)

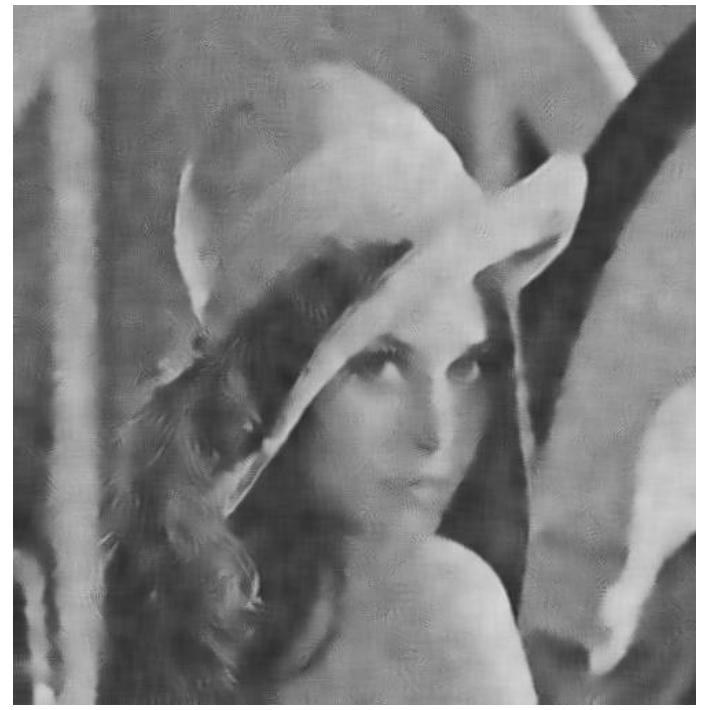

(d)

Figure 4- 9: Subjective comparison for denoising performance for Non-Local Means at noise level $\sigma=80$. (a) Noise free image Lena, (b) noisy image with Additive white Gaussian noise, (c) denoised image using the NLM method, PSNR= 23.05, and (d) denoised image using the proposed method, PSNR $=23.96$. 


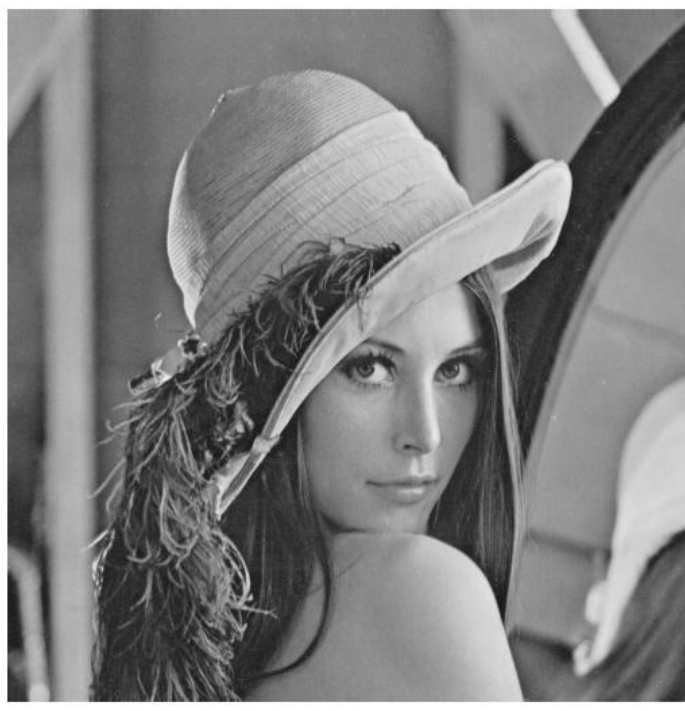

(a)

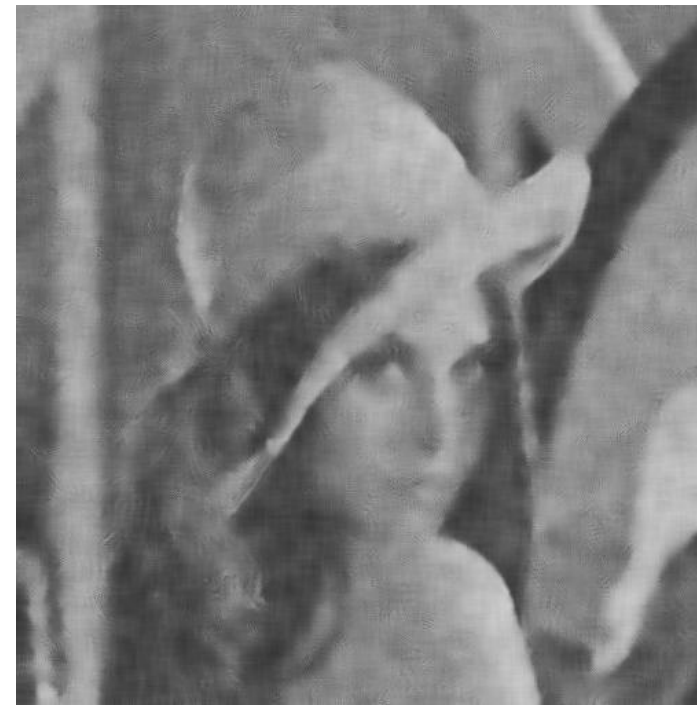

(c)

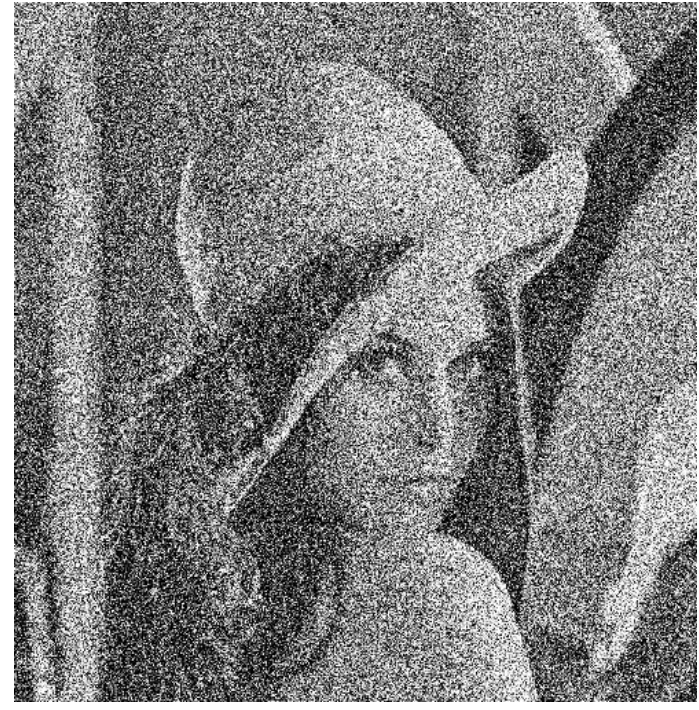

(b)

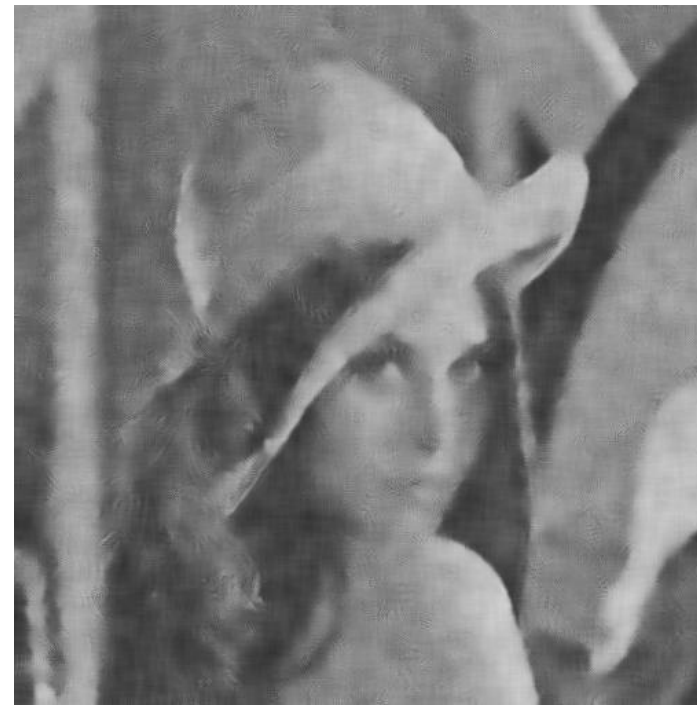

(d)

Figure 4- 10: Subjective comparison for denoising performance for Non-Local Means at noise level $\sigma=100$. (a) Noise free image Lena, (b) noisy image with Additive white Gaussian noise, (c) denoised image using the NLM method, PSNR= 22.18, and (d) denoised image using the proposed method, PSNR $=22.29$. 


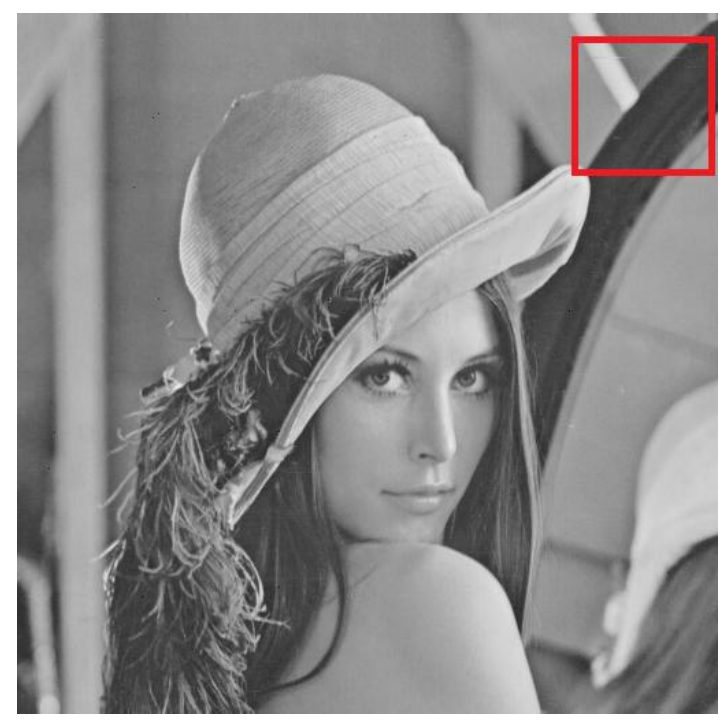

(a)

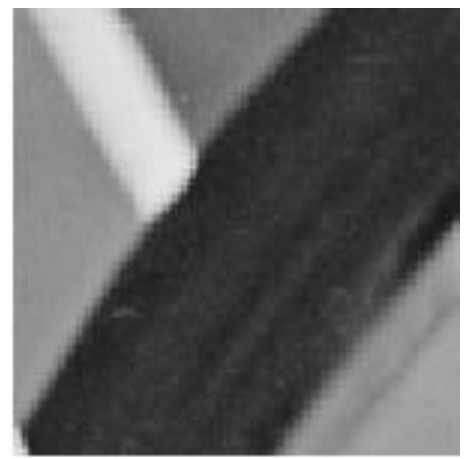

(b)

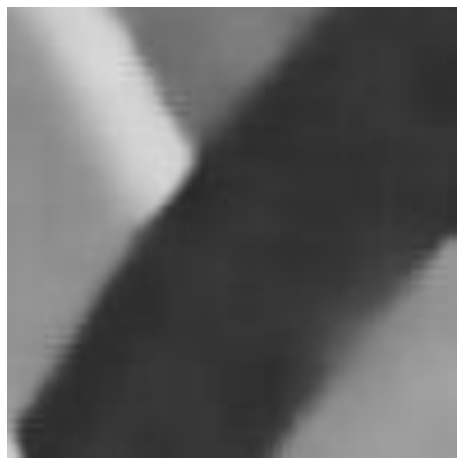

(d)

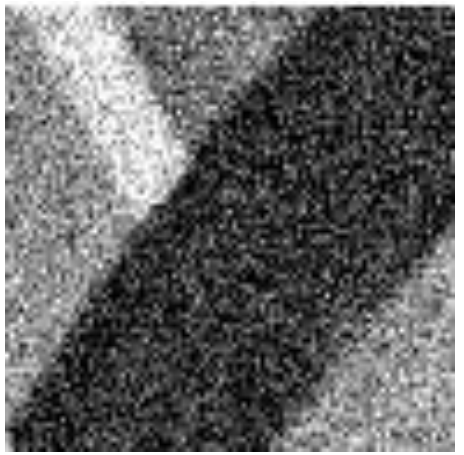

(c)

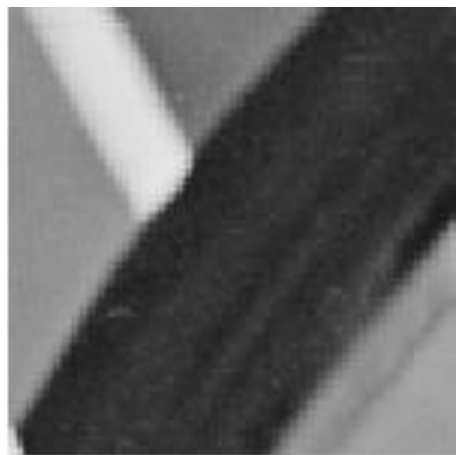

(e)

Figure 4- 11: Output analysis for edge and contrast preservation for Lena image. (a) Original Lena image (b) Noise free fragment of Lena image (c) noisy fragment, $\sigma=40$ (d)denoised fragment using the original NLM method, and (e) denoised fragment using the proposed method. 


\subsubsection{Intensity profile}

The image intensity profile can explain the performance of the proposed method. Figure 4-12 shows the chosen horizontal scan line 50 from the house image. Figure 4-13 and Figure 4-14 show the intensity profile for the true image and the noisy image at noise level 10 and 50, respectively. Figure 4-15, Figure 4-16, Figure 4-17 and Figure 4-18 plot the intensity profile corresponding to different noise levels. In these figures, the blue line represents the true image, the red line represents the denoised image and the green line represents the noisy image. Figure 4-15 and Figure 4-16 show that the proposed method performs better than the original NLM method. Figure 4-17 and Figure 4-18 compares the intensity profile for noise level $\sigma=50$ and it has been found that, at the edge location, the intensity profile of the proposed method is much closer to the noise free image than that of the original NLM method.

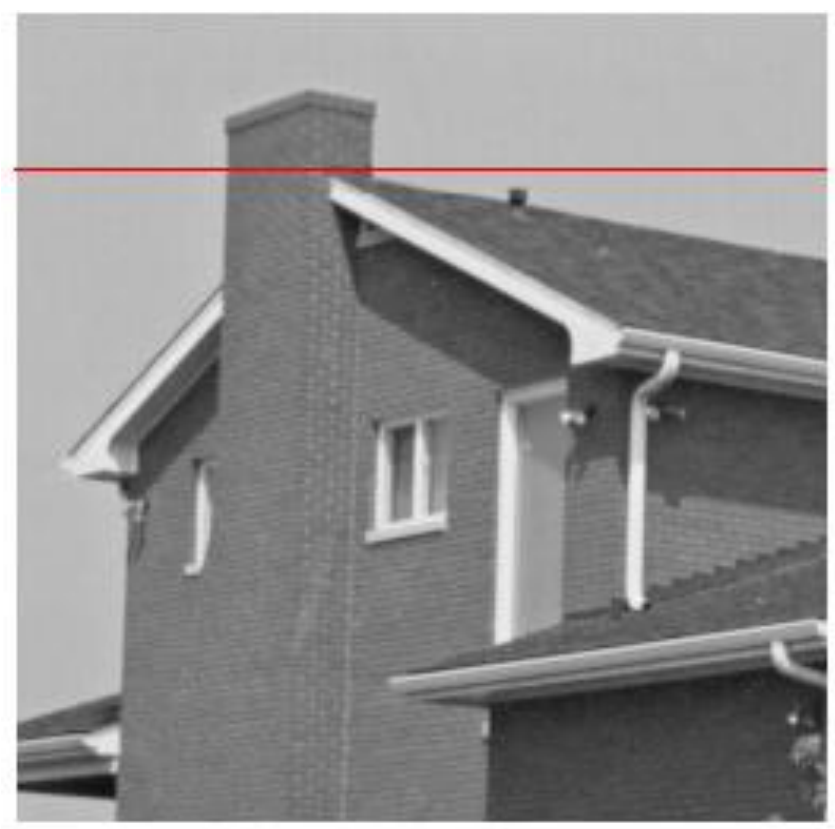

Figure 4- 12: Row number 50 of the House image is chosen as the scan line (dark red horizontal line) to generate intensity profiles. 


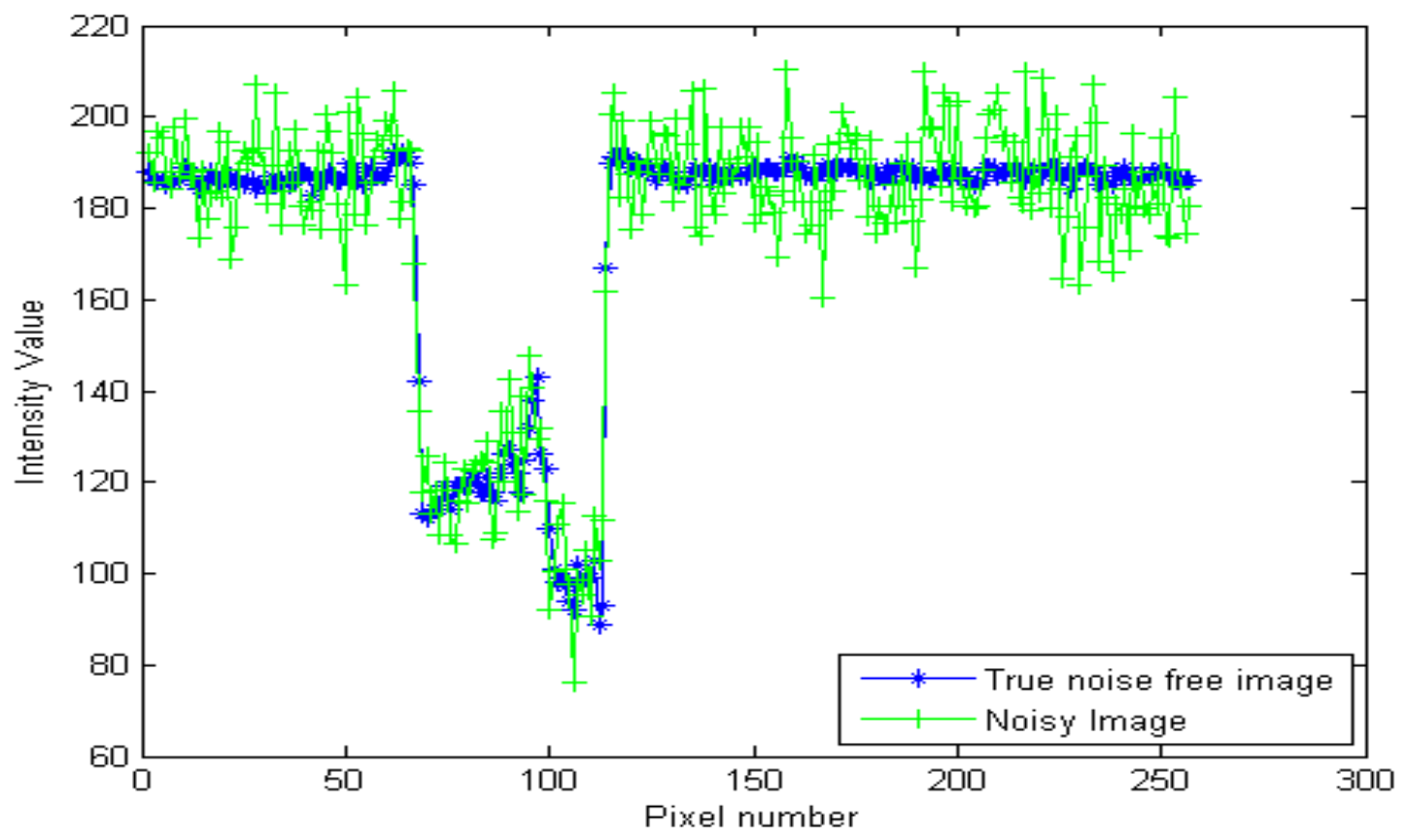

Figure 4- 13: Intensity profile of the House image at scan Line $50(\sigma=10)$.

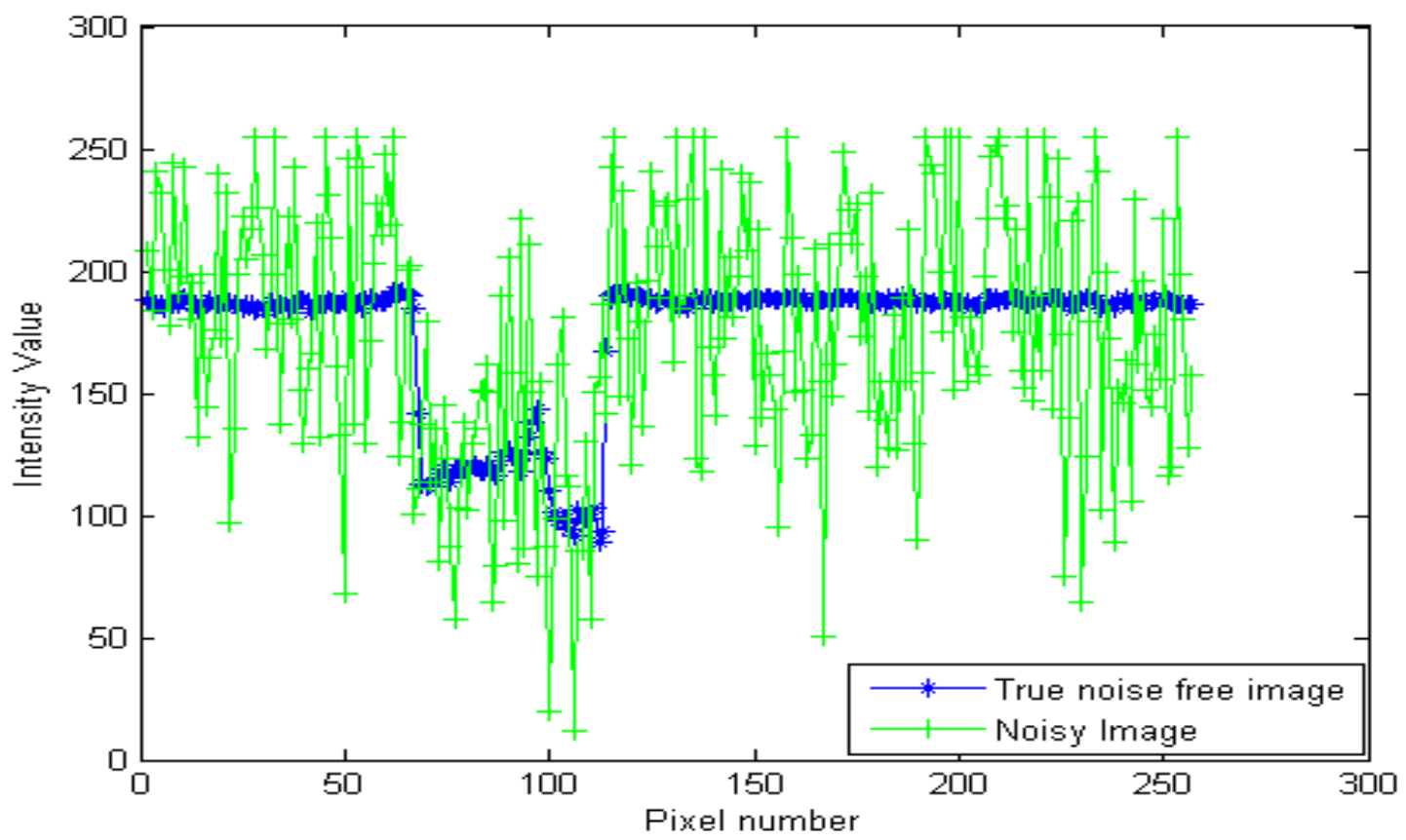

Figure 4- 14: Intensity profile of the House image at scan Line $50(\sigma=50)$. 


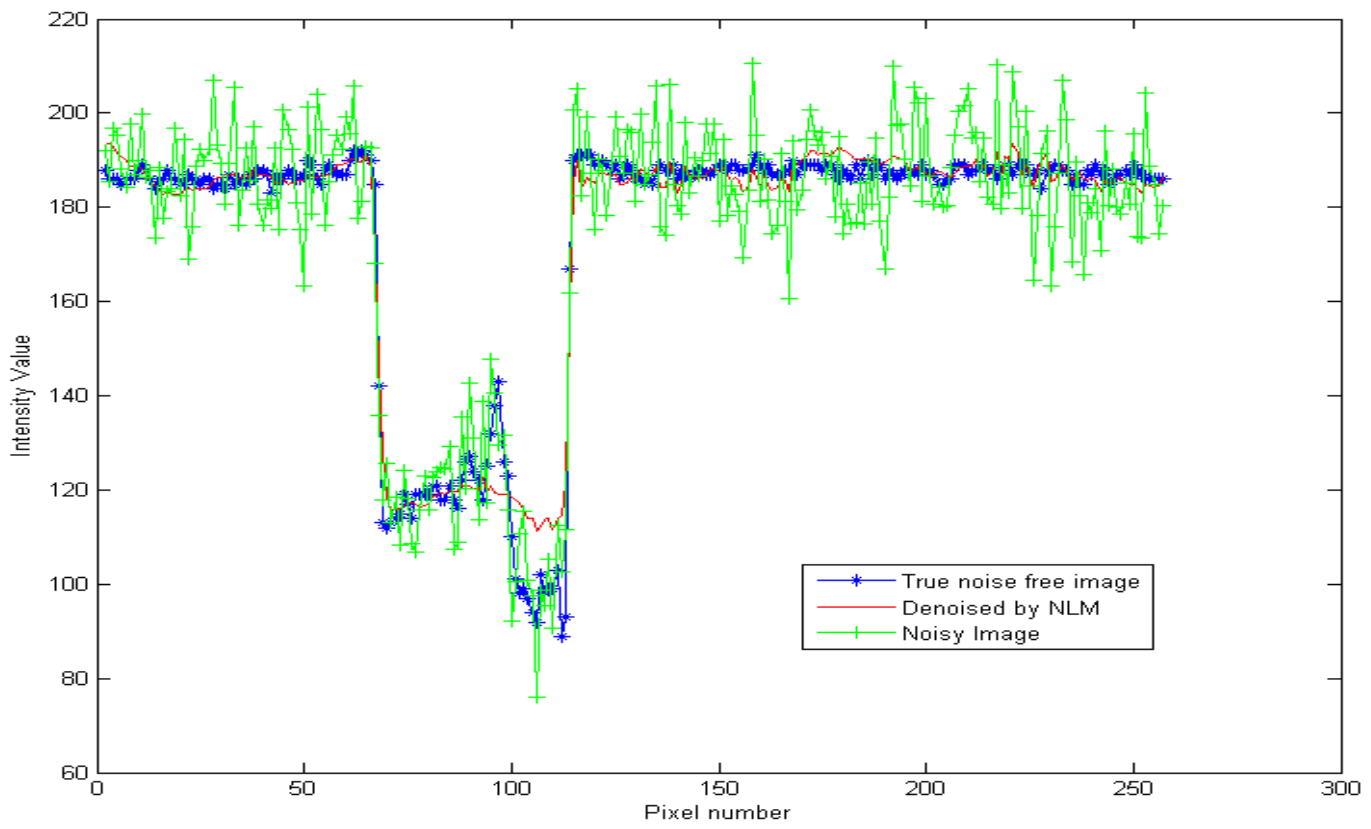

Figure 4- 15: Intensity profile of the House image and denoised image by the NLM method at scan Line $50(\sigma=10)$.

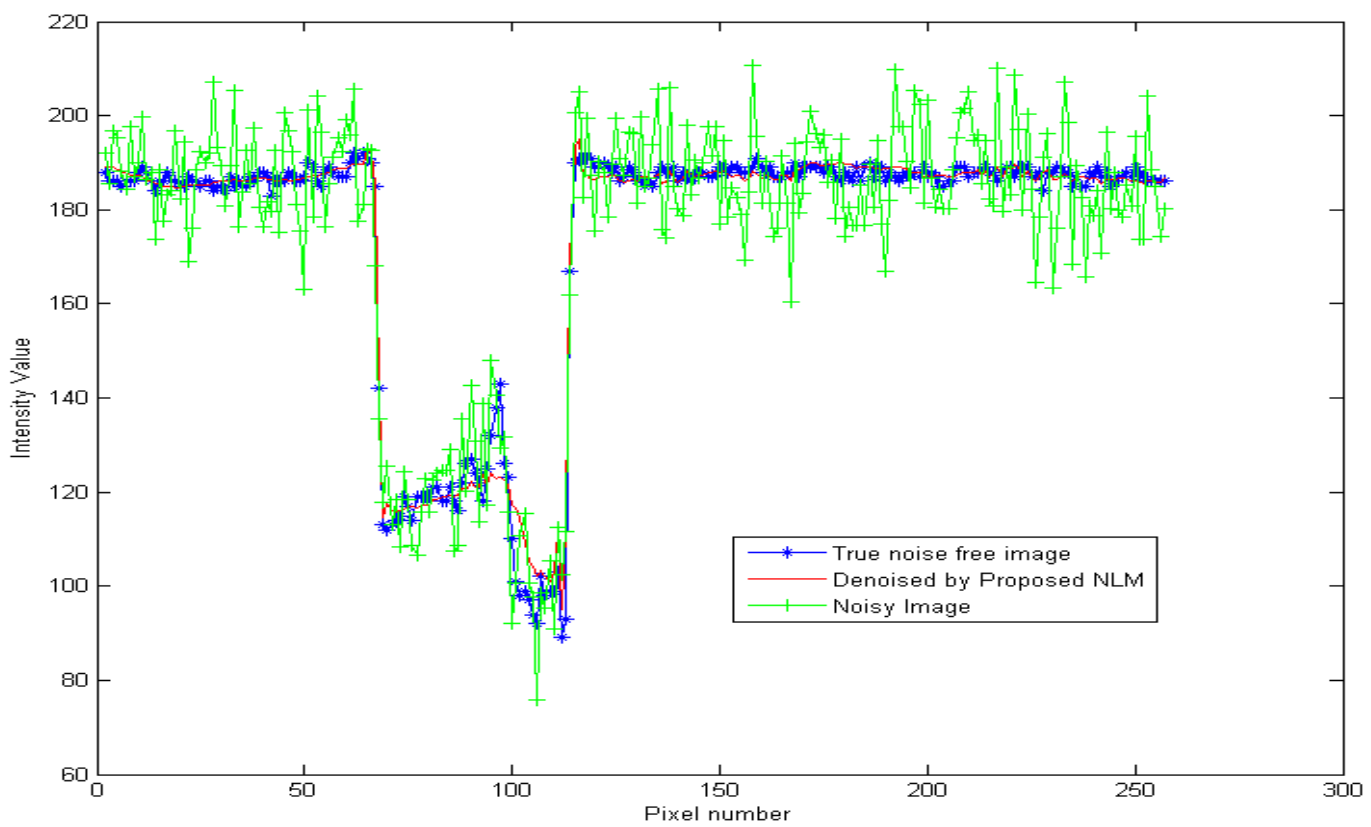

Figure 4- 16: Intensity profile of the House image and denoised image by the proposed method at scan Line $50(\sigma=10)$. 


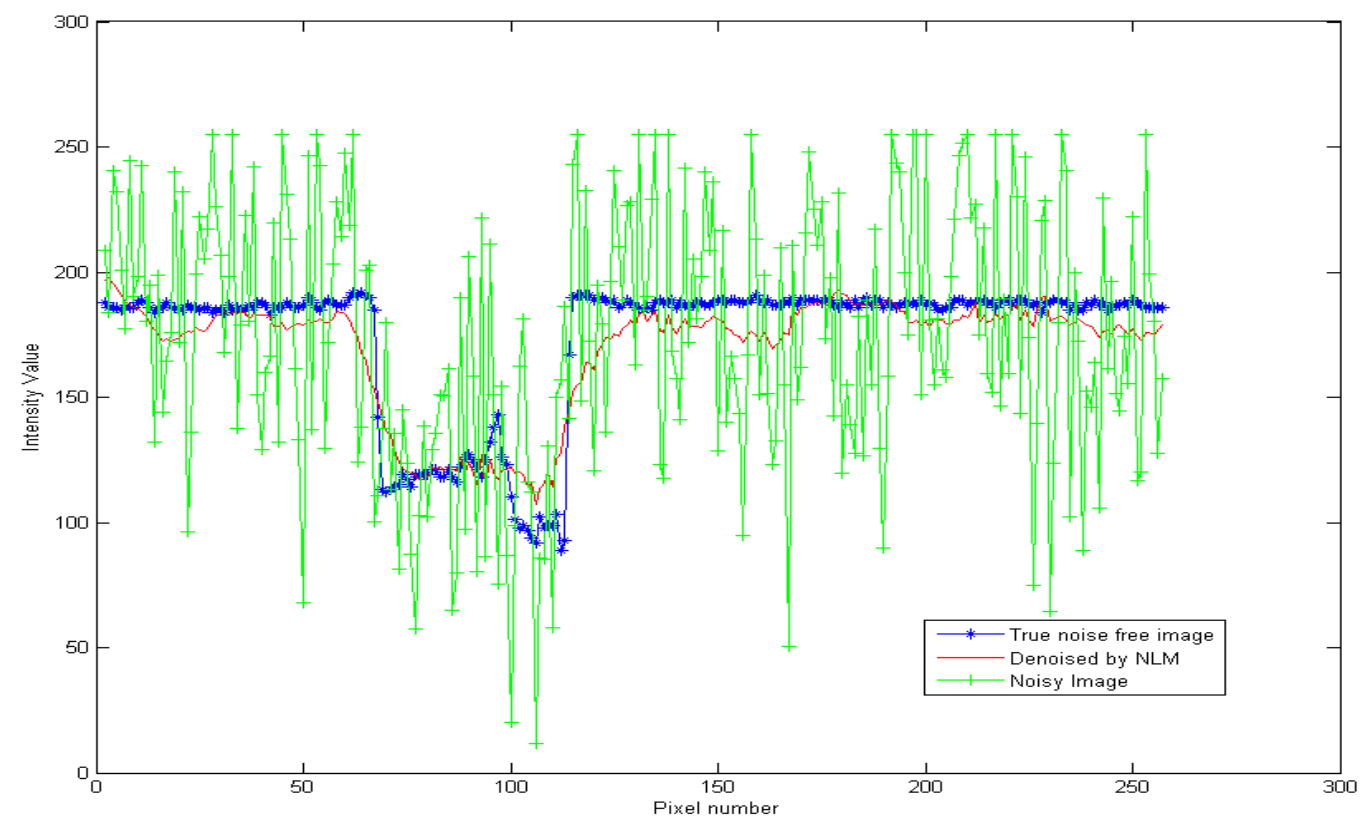

Figure 4- 17: Intensity profile of the House image and denoised image by the NLM at scan Line $50(\sigma=50)$.

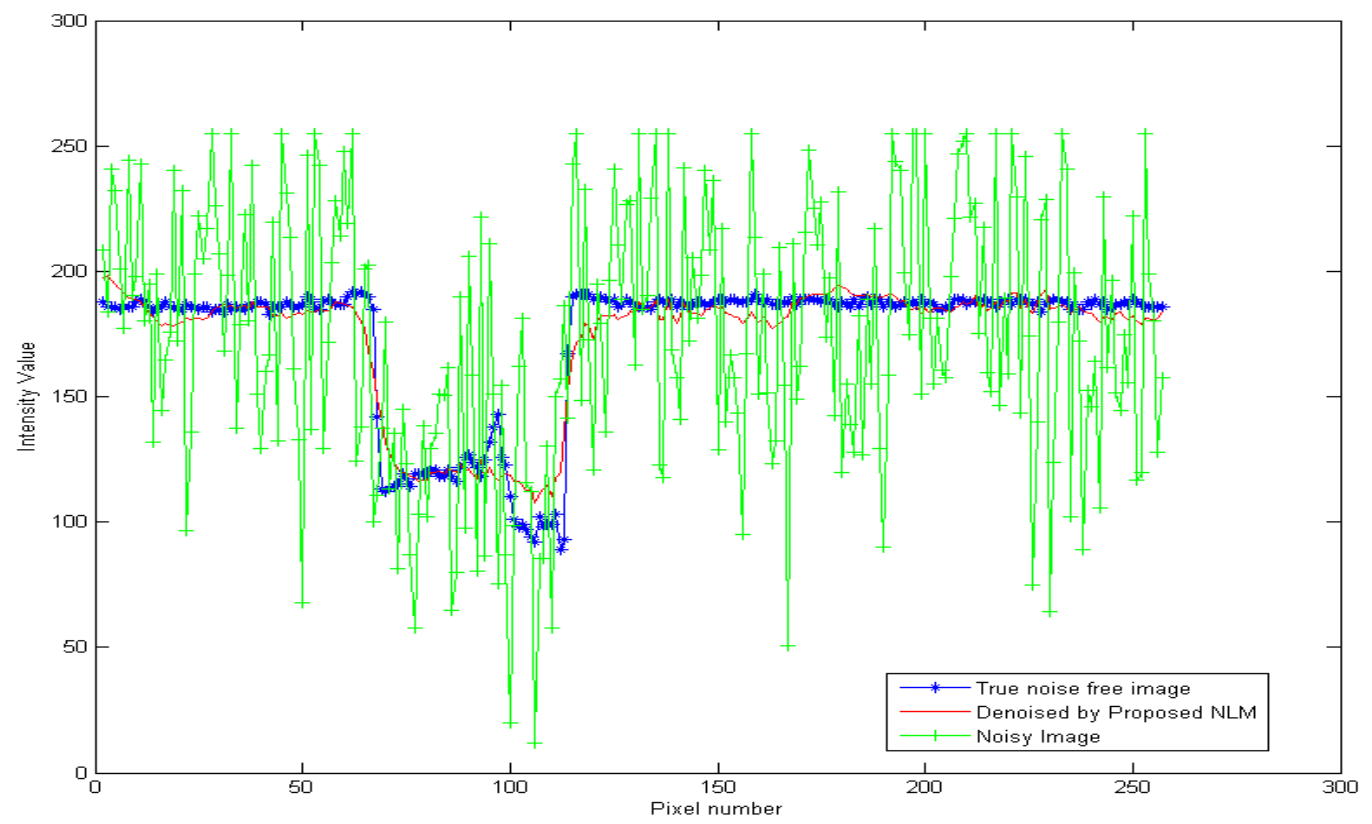

Figure 4- 18: Intensity profile of House image and denoised image by the proposed method at scan Line $50(\sigma=50)$. 


\subsubsection{Summary}

Our proposed method reduces the size of the feature vectors and requires less time over the other versions of the NLM algorithm. Experimental result shows that the proposed method performs the best in terms of running time among the other denoisng algorithms and provides better performance in terms of PSNR and SSIM. In visual quality comparison, it is also clear that it shows a better performance over the original NLM. Result was simulated using different noise levels. To compare the performance, we have tested our method on different test images. In all of the cases, it performs better than the NLM and the variants of NLM. Yet, it performs better than BM3D for lower noise levels and performs close to BM3D for higher noise levels.

Visual quality comparison clearly shows that our proposed method reduces noise and preserves fine edges over the original NLM method for all of the test cases. Also it reduces artifacts and gives better denoisng for all of the test cases.

In summary, it shows:

- Better performance in running time.

- Better preservation of edges.

- Fewer artifacts on denoising and exploits fine details.

- Better PSNR and SSIM values over the original NLM method. 


\section{Chapter 5}

\section{Conclusion and Future Work}

\subsection{Conclusion}

Non-Local Means is a popular image denoising algorithm implemented in the spatial domain. In this thesis we have proposed a statistics based improvement for the Non-Local Means algorithm. The key of this improvement is to reduce the size of the feature space, which reduces the patch similarity measurement time and increases the overall denoising performance. We have utilized a statistical t-test to reduce the dimensionality of the feature space. This reduced feature space is used during the denoising process.

The proposed method has three parameters. The patch size, the search region size and the threshold value for the $t$-test. We optimized these parameters on a set of test images. The optimized parameters are used in our proposed method to improve the performance of the denoising scheme.

We have extensively tested and analyzed our proposed method using both objective and subjective measures. We have compared the proposed method with the original NonLocal Means algorithm and its variants. We have also compared the proposed method with the BM3D image denoising algorithm. Experimental results show that our proposed method provides the best running time among all other algorithms in all test cases at various noise levels. It also provides a good denoising improvement in terms of the PSNR and the SSIM values. In addition, it performs better than the NLM method and its variants at all noise levels and perform better than the BM3D method for lower noise levels. However, the BM3D method performs better at the higher noise levels.

We have also showed visual quality comparisons for various test images. It has been

found that the proposed method performs better than the original NLM method in visual quality comparison. 
To evaluate the performance in terms of edge and contrast preservation, we have tested the proposed method on a fragment of Lena image. It has been found that our proposed method performs better than the original NLM method. This finding has been demonstrated and confirmed by comparing intensity profiles from the output of the proposed method and the NLM method.

Finally, we can conclude that the proposed method performs better than the NLM method and its variants for all test cases. Moreover, it performs better than the BM3D method for lower noise levels. However, the BM3D method performs better at higher noise levels.

\subsection{Future Work}

Our proposed scheme can be extended to video data. In that case, denoising a pixel will depend on the reference patch at time frame $t$ and also on the same patch at the previous time frame $t-1$. Thus more pixels can contribute into the denoising process.

Color image denoising can also be considered as a future work. Instead of denoising the intensity value of the noisy pixel, luminance and chrominance information can be considered to denoise a color pixel. The proposed method can also be implemented in different applications. 


\section{References}

[1] G. Portilla, J. Strela, V. Wainwright, and M. Simoncelli, Image denoising using scale mixtures of gaussians in the wavelet domain. IEEE Transactions on Image Processing 12(11), 1338-1351, 2003.

[2] L. Yuan, J. Sun, L. Quan, and H. Shum, Progressive interscale and intra-scale non-blind image deconvolution, ACM Transactions on Graphics 27(3), 1-10, 2008.

[3] S. Roth and M. Black, Fields of experts: A framework for learning image priors, IEEE Computer Society Conference on Computer Vision and Pattern Recognition, pp. 860-867, 2005.

[4] A. Buades, B. Coll, and J-M. Morel, A non-local algorithm for image denoising, IEEE Computer Society Conference on Computer Vision and Pattern Recognition, pp. 60-65, 2005.

[5] K. Dabov, A. Foi, V. Katkovnik, and K. Egiazarian, Image denoising by sparse 3-D transform-domain collaborative filtering, IEEE Transactions on Image Processing, 16(8), pp. 2080-2095, 2007.

[6] K. Chaudhury, and A. Singer, Non-local patch regression: Robust image denoising in patch space, IEEE International Conference on Acoustics, Speech, and Signal Processing (ICASSP), pp. 1345 1349, 2013.

[7] K. Chaudhury, and A. Singer, Non-local Euclidean medians, IEEE Signal Processing Letters, vol. 19(11), pp. 745 - 748, 2012.

[8] R. Vignesh, B. Oh, and C.-C. Kuo, Fast Non-Local Means (NLM) Computation With Probabilistic Early Termination, IEEE Signal Processing Letters, 17(3), pp.277-280, 2010.

[9] H. Bhujle, and S. Chaudhuri, Novel Speed-Up Strategies for Non-Local Means Denoising With Patch and Edge Patch Based Dictionaries, IEEE Transactions on Image Processing, 23(1), pp.356-365, 2014.

[10]M. Mahmoudi, and G. Sapiro, Fast image and video denoising via nonlocal means of similar neighborhoods, IEEE Signal Processing Letters, 12(12), pp.839-842, 2005.

[11]P. Chatterjee and P. Milanfar, Is denoising dead?, IEEE Transactions on Image Processing, 19(4), pp. 895-911, 2010.

[12]T. Tasdizen, Principal components for non-local means image denoising, 15th IEEE International Conference on Image Processing, pp.1728-1731, 2008.

[13]T. Brox, O. Kleinschmidt and D. Cremers, Efficient Nonlocal Means for Denoising of Textural Patterns, IEEE Transactions on Image Processing, 17(7), pp.1083-1092, 2008.

[14]H. Kwan, Intelligent digital filters with application to salt and pepper noise reduction in MR brain images. 2013 Constantinides International Workshop on Signal Processing, pp. 1-4, 2013.

[15]M. Iftikhar, S. Rathore, A. Jalil and M. Hussain. A novel extension to non-local means algorithm: Application to brain MRI de-noising. 2013 16th International Multi Topic Conference, pp. 195-200, 2013. 
[16]Z. Kelm, D. Blezek, B. Bartholmai and B. Erickson. Optimizing non-local means for denoising low dose CT, IEEE International Symposium on Biomedical Imaging: From Nano to Macro, pp. 662-665, 2009.

[17] S. Hu and W. Hou. Denosing 3D ultrasound images by non-local means accelerated by GPU, 2011 International Conference on Intelligent Computation and Bio-Medical Instrumentation, pp. 43-45, 2011.

[18]X. Lian and T. Wang. Restoration of medical ultrasound images via modified homomorphic deconvolution, 5th International Conference on Visual Information Engineering, pp.504-507, 2008.

[19] A. Rudnitskii. Separating heart sound from lung sound using non-local means filter, 2014 IEEE 34th International Conference on Electronics and Nanotechnology, pp. 361-364, 2014 .

[20] V. Basavaraja, A. Bopardikar and S. Velusamy, Detail warping based video super-resolution using image guides. 17th IEEE International Conference on Image Processing, pp. 2009-2012, 2010.

[21]H. Seo and P. Milanfar. Video denoising using higher order optimal space-time adaptation, IEEE International Conference on Acoustics, Speech and Signal Processing, pp. 1249-1252, 2008.

[22]Q. Xu, H. Jiang, R. Scopigno and M. Sbert. A new approach for very dark video denoising and enhancement, 17th IEEE International Conference on Processing, pp. 1185-1188, 2010.

[23] G.Martino, G. Poggi, D. Riccio and L. Verdoliva. Effects of despeckling on the estimation of fractal dimension from SAR images, 2013 IEEE International Geoscience and Remote Sensing Symposium, pp. 3950-3953, 2013 .

[24]Z. Hongyu, W. Quan, W. Weiwei, W. Qingping and Y. Naichang. SAR image despeckling based on improved non-local means algorithm, 2014 International Conference on Electromagnetics in Advanced Applications, pp. 844-847, 2014.

[25] Y. Zhao and Y. Liu. Patch based saliency detection method for 3D surface simplification. 21st International Conference on Pattern Recognition, pp. 845-848, 2012 .

[26] Y. Zhao, Y. Liu, R. Song and M. Zhang. Extended non-local means filter for surface saliency detection. 19th IEEE International Conference on Image Processing, pp. 633-636, 2012.

[27] A. Mouton, N. Megherbi, G. T. Flitton, S. Bizot and T. P. Breckon. A novel intensity limiting approach to metal artefact reduction in 3D CT baggage imagery, 19th IEEE International Conference on Image Processing, pp. 2057-2060, 2012.

[28] M. Salmeri, A. Mencattini, E. Ricci, and A. Salsano, Noise estimation in digital images using fuzzy processing, 2001 International Conference on Image Processin, pp. 517-520, 2001.

[29]D. Shin, R. Park, S. Yang, and J. Jung, Block-based noise estimation using adaptive gaussian filtering, IEEE Transactions on Consumer Electronics, 51(1), pp. 218-226, 2005. 
[30] R. Bilcu and M. Vehvilainen, New method for noise estimation in images, IEEE-Eurasip in Nonlinear Signal and Image Processing, p. 25-28. 2005.

[31] F. Russo, Gaussian noise estimation in digital images using nonlinear sharpening and genetic optimization, IEEE Instrumentation and Measurement Technology Conference, pp. 1-5, 2007.

[32] A. De Stefano, P. White, and W. Collis, Training methods for image noise level estimation on wavelet components, EURASIP Journal on Applied Signal Processing, pp. 2400-2407, 2004.

[33] S. Aja-Fernandez, G. Ferrero, M. Mart', Fernandez, and C. Alberola-Lopez, Automatic noise estimation in images using local statistics. additive and multiplicative cases, Image and Vision Computing, 27(6), pp. 756-770, 2009. 


\section{Appendices}

Appendix A

PSNR, SSIM and Subjective comparison

Table A- 1: PSNR (dB) comparison for Peppers image among the proposed method, the NLM method, variants of the NLM method and the BM3D method for different noise levels.

\begin{tabular}{|c|c|c|c|c|c|}
\hline Noise Level & NLM & PCA-NLM & NLM-Patch & $\begin{array}{l}\text { Proposed } \\
\text { Method } \\
\end{array}$ & BM3D \\
\hline \multicolumn{6}{|c|}{ Peppers } \\
\hline 10 & 33.24 & 33.72 & 33.52 & 35.01 & 34.68 \\
\hline 20 & 31.29 & 30.95 & 30.41 & 31.70 & 31.29 \\
\hline 30 & 29.72 & 29.84 & 27.64 & 30.19 & 29.28 \\
\hline 40 & 28.26 & 28.40 & 25.71 & 29.74 & 27.70 \\
\hline 50 & 26.93 & 27.07 & 25.12 & 28.40 & 26.68 \\
\hline 60 & 24.24 & 25.01 & 23.96 & 25.77 & 25.81 \\
\hline 70 & 23.41 & 23.59 & 23.07 & 23.60 & 25.07 \\
\hline 80 & 23.31 & 23.24 & 22.52 & 23.58 & 24.45 \\
\hline 90 & 22.54 & 22.67 & 21.98 & 22.61 & 23.87 \\
\hline 100 & 21.48 & 21.39 & 21.13 & 21.77 & 23.39 \\
\hline Average & 26.44 & 26.59 & 25.50 & 27.23 & 27.24 \\
\hline
\end{tabular}


Table A- 2: SSIM comparison for Peppers image among the proposed method, the NLM method, variants of the NLM method and the BM3D method for different noise levels.

\begin{tabular}{|c|c|c|c|c|c|}
\hline Noise Level & NLM & PCA-NLM & $\begin{array}{l}\text { NLM- } \\
\text { Patch } \\
\end{array}$ & $\begin{array}{c}\text { Proposed } \\
\text { Method }\end{array}$ & BM3D \\
\hline \multicolumn{6}{|c|}{ Peppers } \\
\hline 10 & 0.9121 & 0.9095 & 0.9042 & 0.9312 & 0.9259 \\
\hline 20 & 0.8791 & 0.8692 & 0.8665 & 0.8837 & 0.8789 \\
\hline 30 & 0.8411 & 0.8394 & 0.8367 & 0.8475 & 0.8432 \\
\hline 40 & 0.8096 & 0.8084 & 0.8062 & 0.8124 & 0.8094 \\
\hline 50 & 0.7555 & 0.7534 & 0.7511 & 0.7678 & 0.7784 \\
\hline 60 & 0.7384 & 0.7392 & 0.7403 & 0.7412 & 0.7467 \\
\hline 70 & 0.7274 & 0.7212 & 0.7191 & 0.7313 & 0.7365 \\
\hline 80 & 0.7106 & 0.7092 & 0.7068 & 0.7162 & 0.7321 \\
\hline 90 & 0.7023 & 0.6982 & 0.7017 & 0.7092 & 0.7242 \\
\hline 100 & 0.6831 & 0.6659 & 0.6623 & 0.6974 & 0.7119 \\
\hline Average & 0.7964 & 0.79098 & 0.7882 & 0.80468 & 0.80883 \\
\hline
\end{tabular}




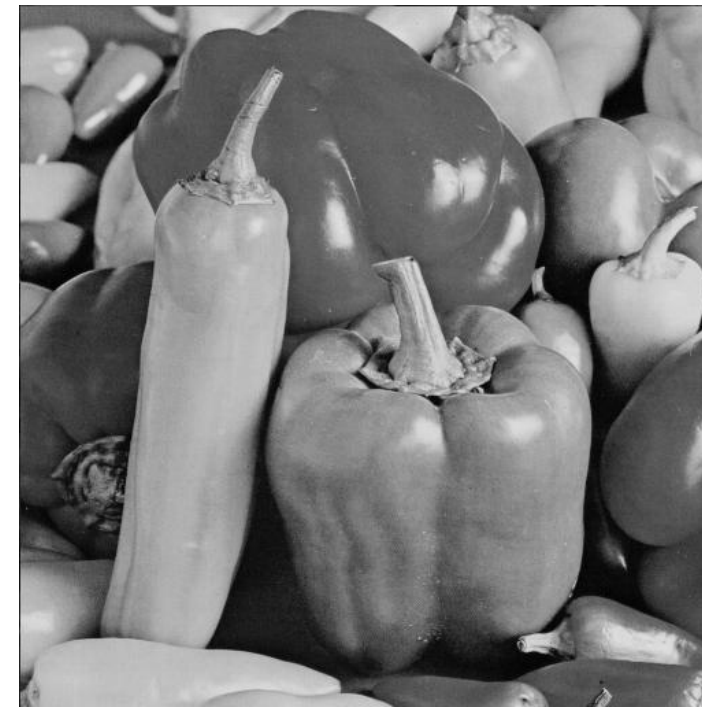

(a)

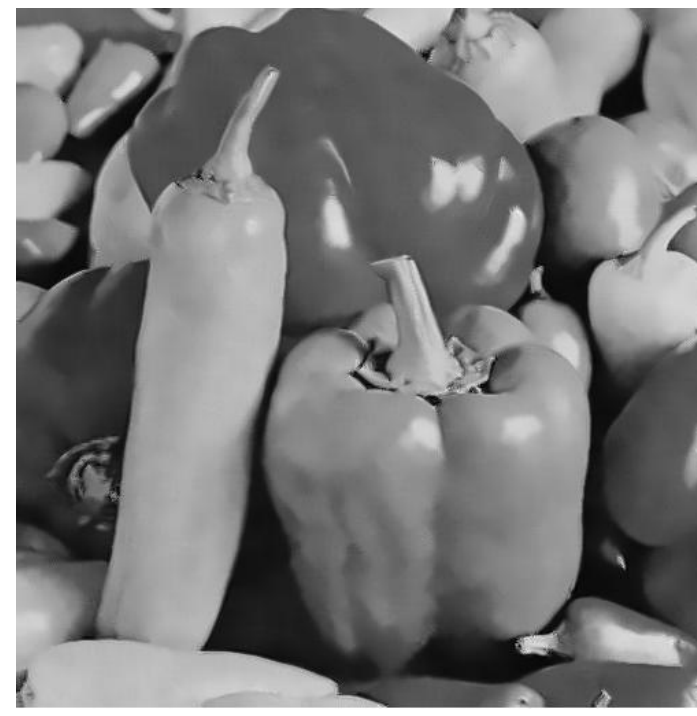

(c)

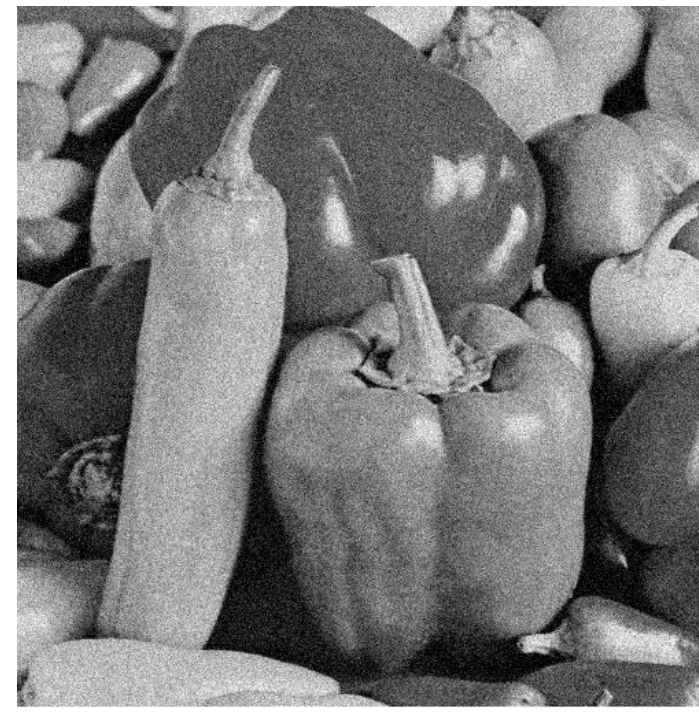

(b)

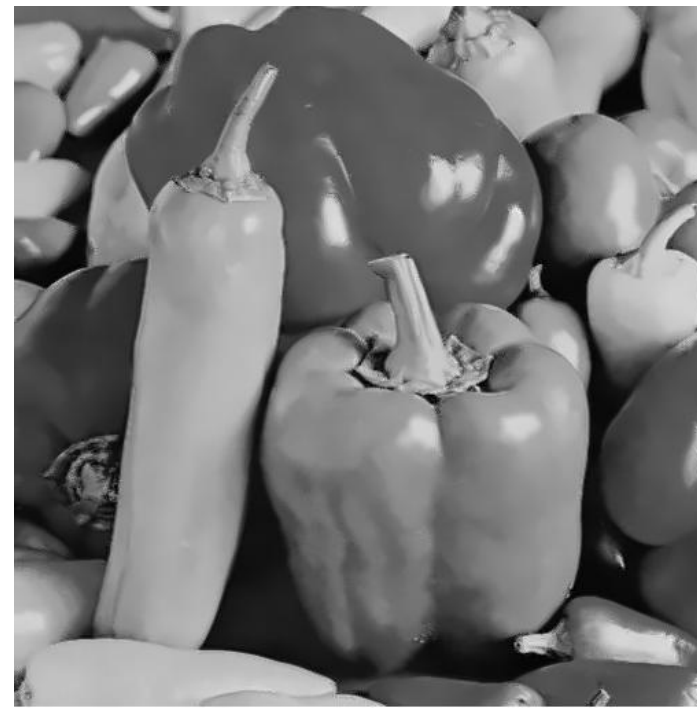

(d)

Figure A- 1: Subjective comparison for denoising performance for Non-Local Means at noise level $\sigma=20$. (a) noise free image Peppers. (b) noisy image with Additive white Gaussian noise. (c) denoised image using the NLM method, PSNR= 31.2973, and (d) denoised image using the proposed method, PSNR $=31.7027$. 


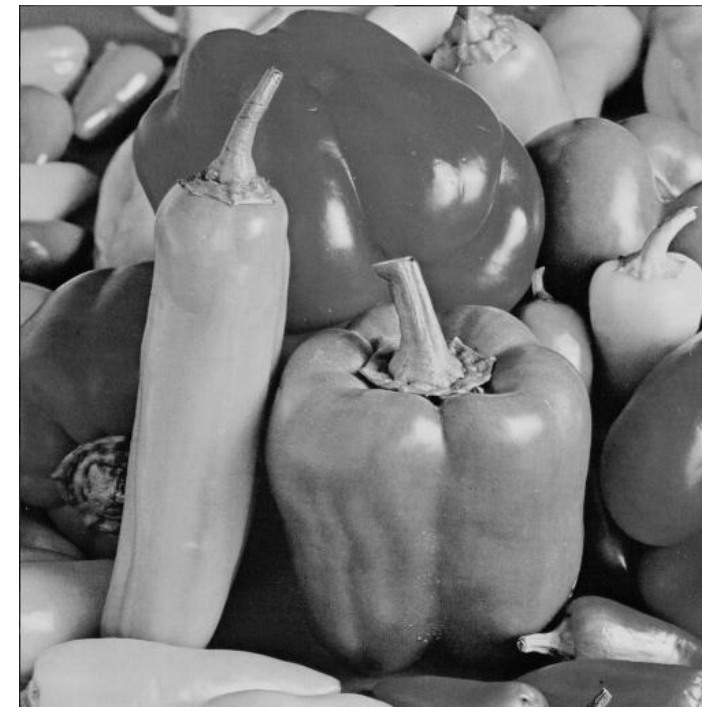

(a)

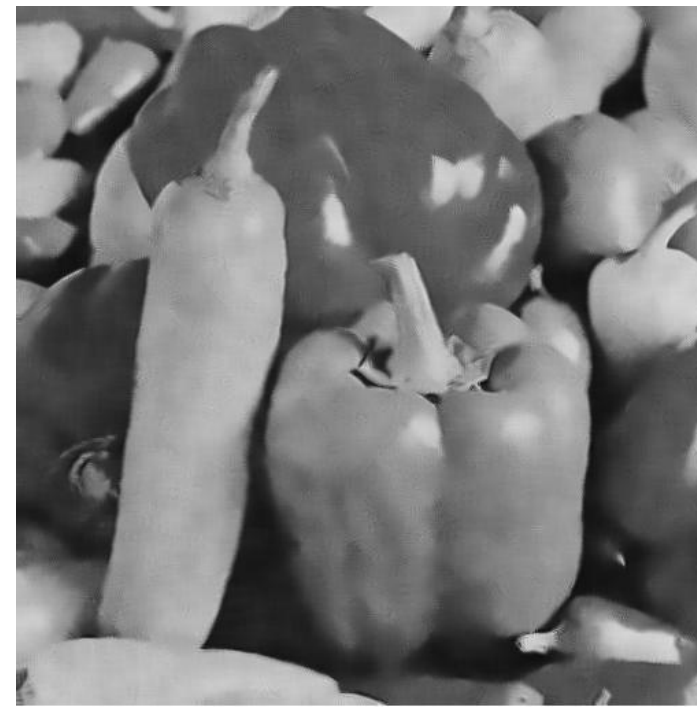

(c)

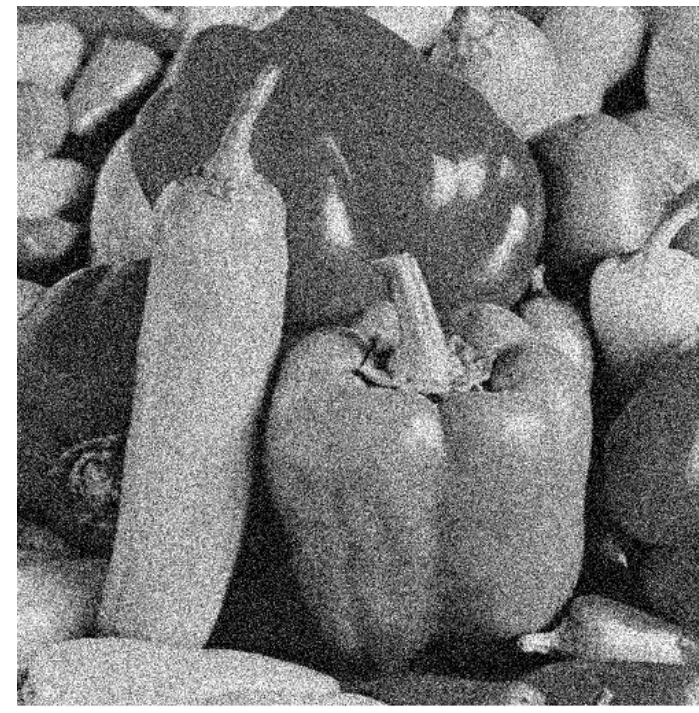

(b)

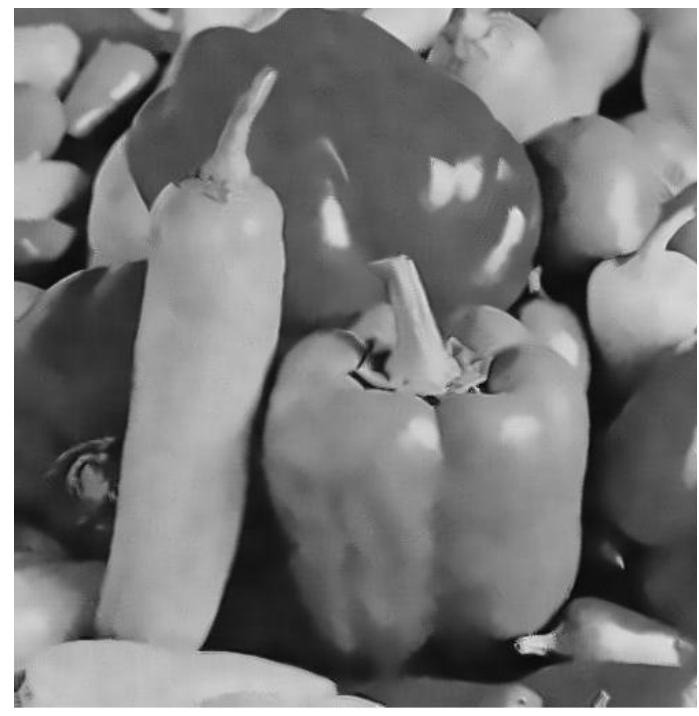

(d)

Figure A- 2: Subjective comparison for denoising performance for Non-Local Means at noise level $\sigma=40$. (a) noise free image Peppers. (b) noisy image with Additive white Gaussian noise. (c) denoised image using the NLM method, PSNR= 28.2603, and (d) denoised image using the proposed method, PSNR $=29.7362$. 


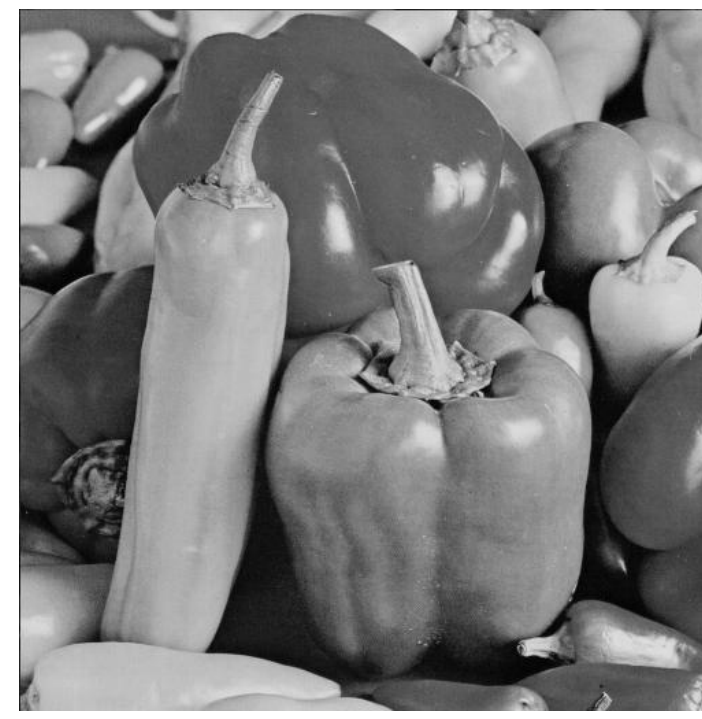

(a)

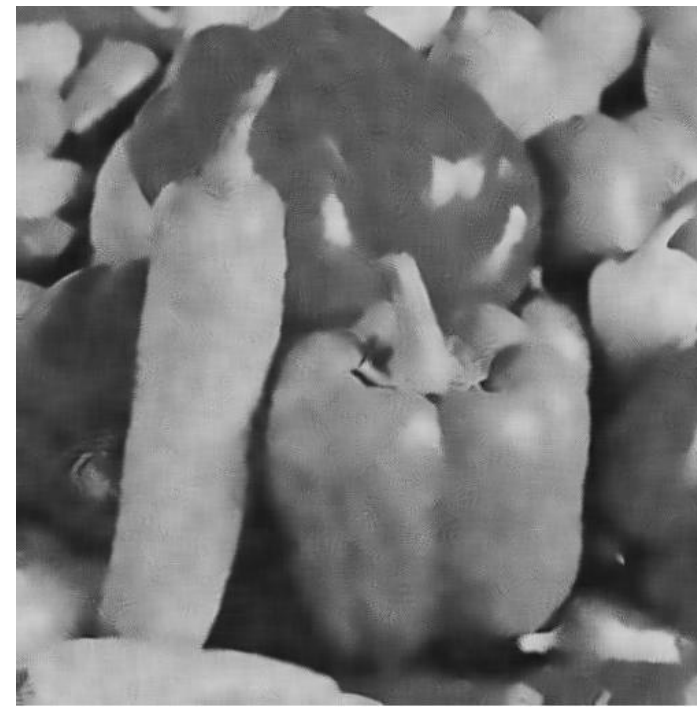

(c)

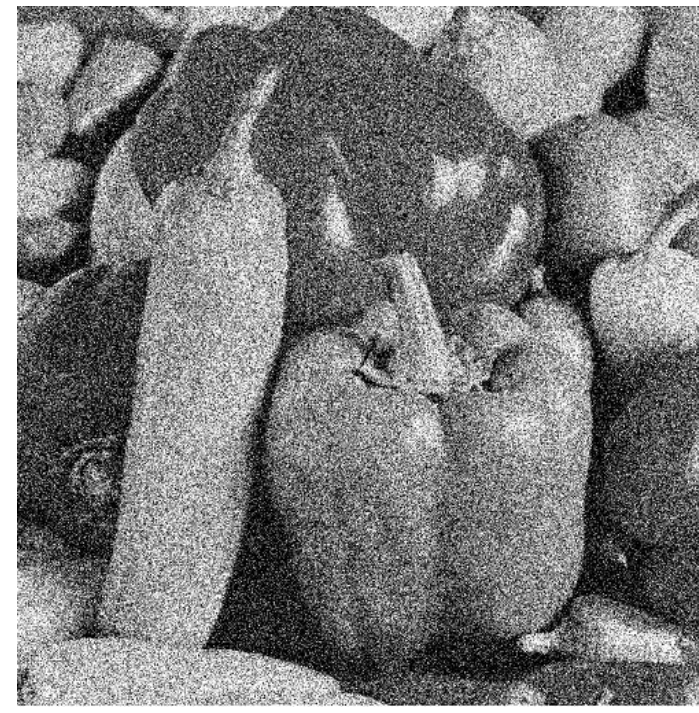

(b)

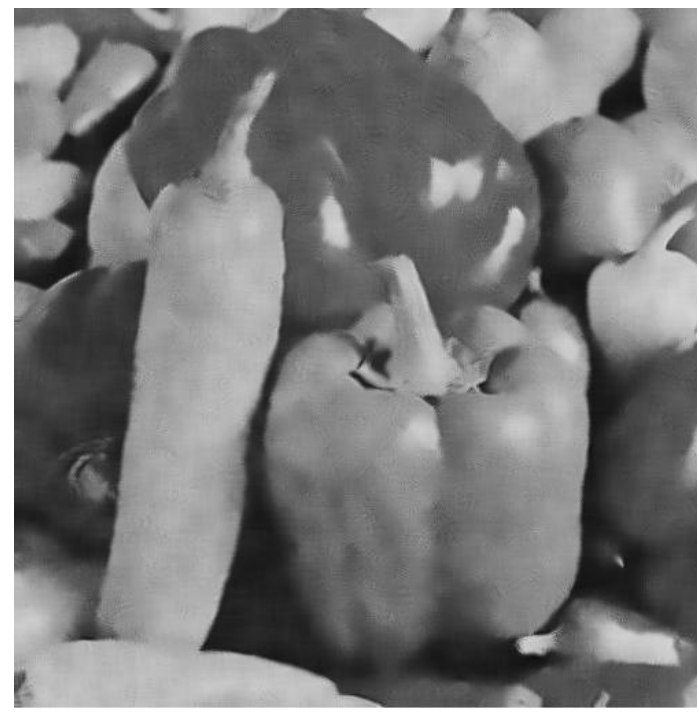

(d)

Figure A- 3: Subjective comparison for denoising performance for Non-Local Means at noise level $\sigma=60$. (a) noise free image Peppers. (b) noisy image with Additive white Gaussian noise. (c) denoised image using the NLM method, PSNR= 24.2415, and (d) denoised image using the proposed method, PSNR $=25.7656$. 


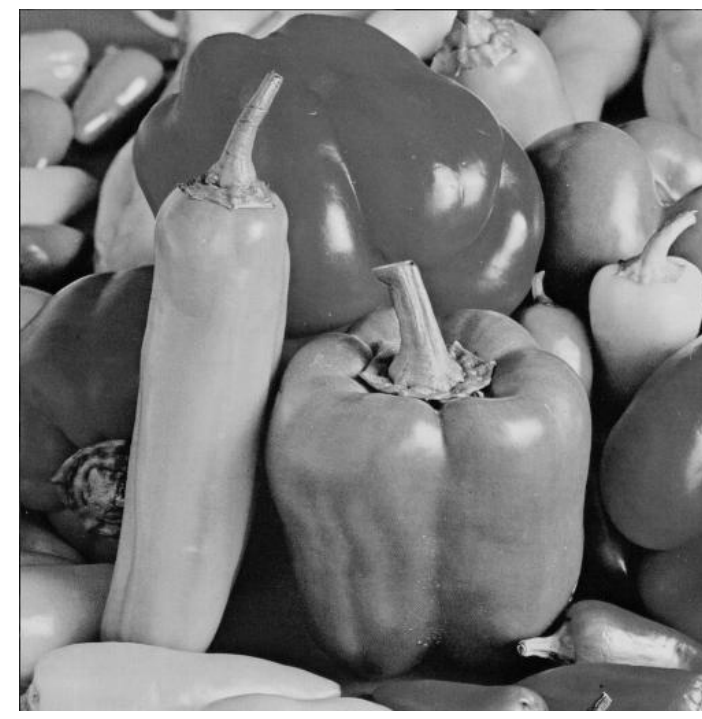

(a)

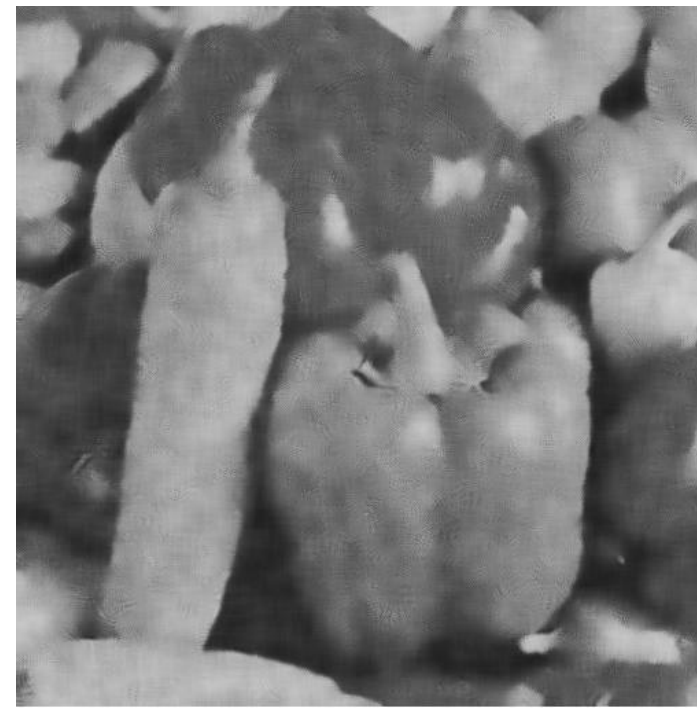

(c)

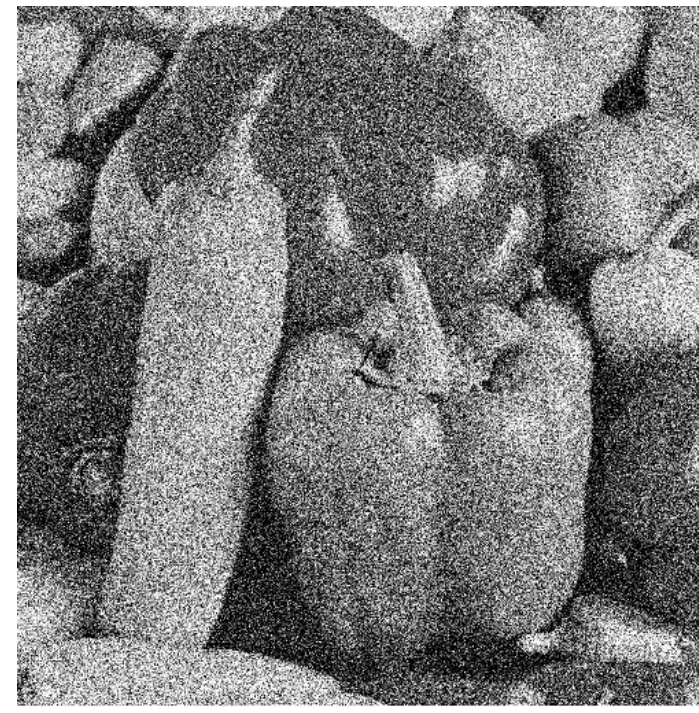

(b)

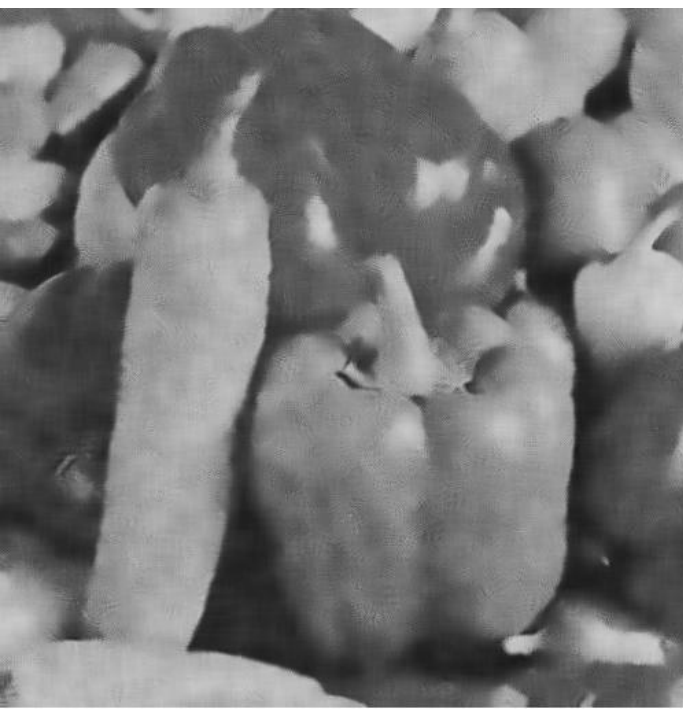

(d)

Figure A- 4: Subjective comparison for denoising performance for Non-Local Means at noise level $\sigma=80$. (a) noise free image Peppers. (b) noisy image with Additive white Gaussian noise. (c) denoised image using the NLM method, PSNR= 23.3125, and (d) denoised image using the proposed method, PSNR $=23.5756$. 


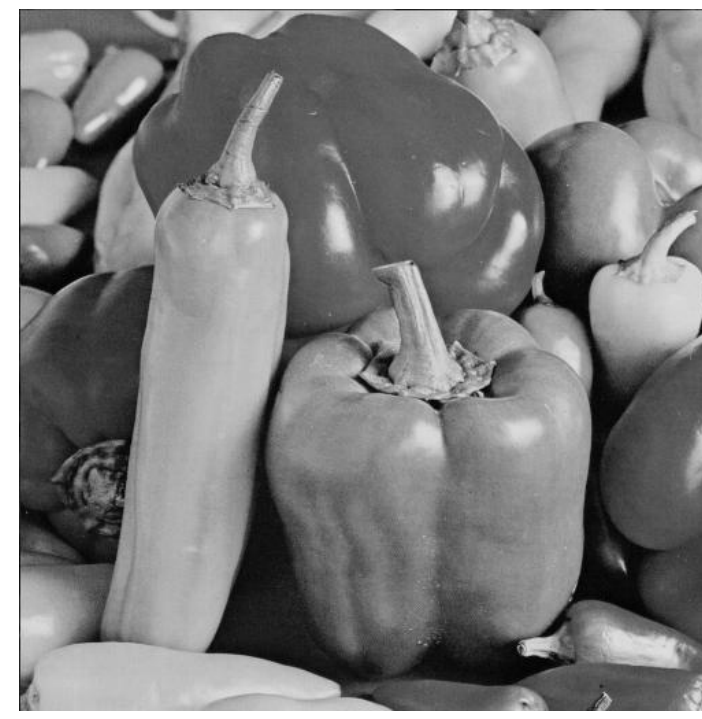

(a)

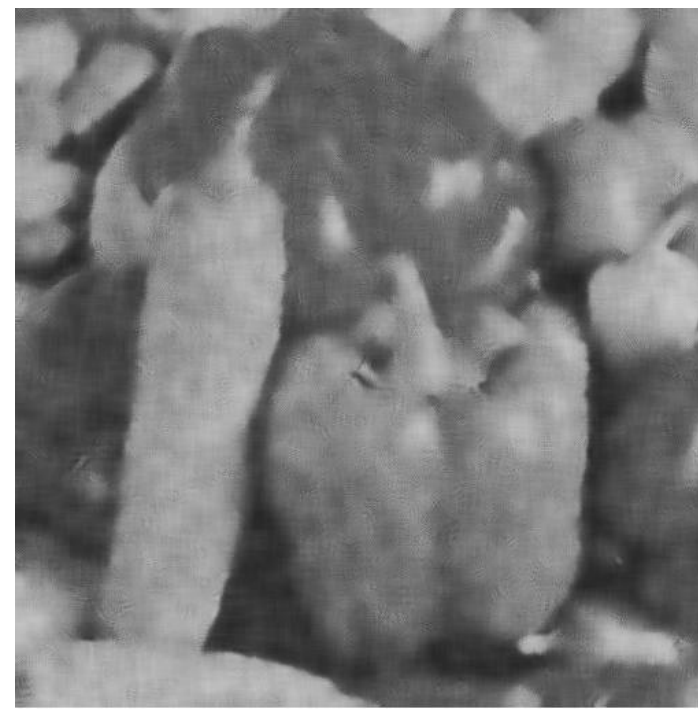

(c)

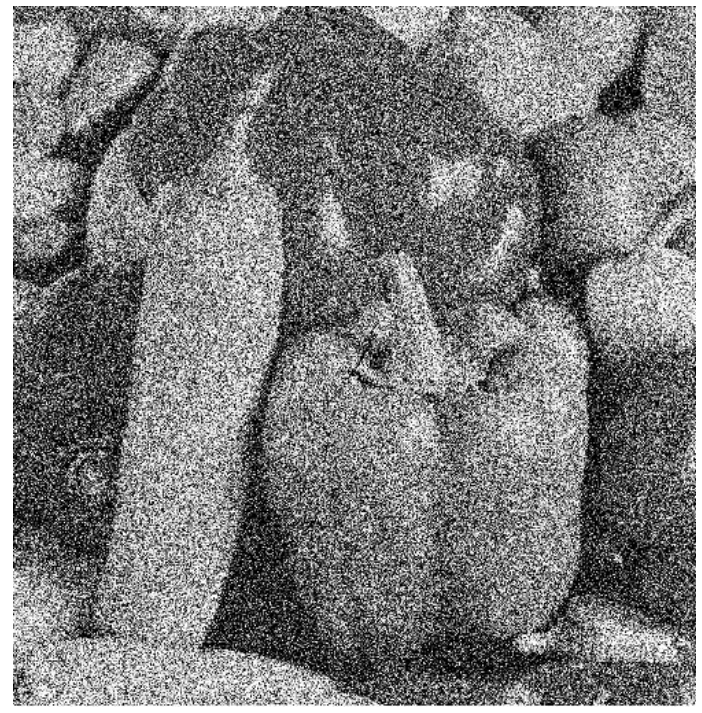

(b)

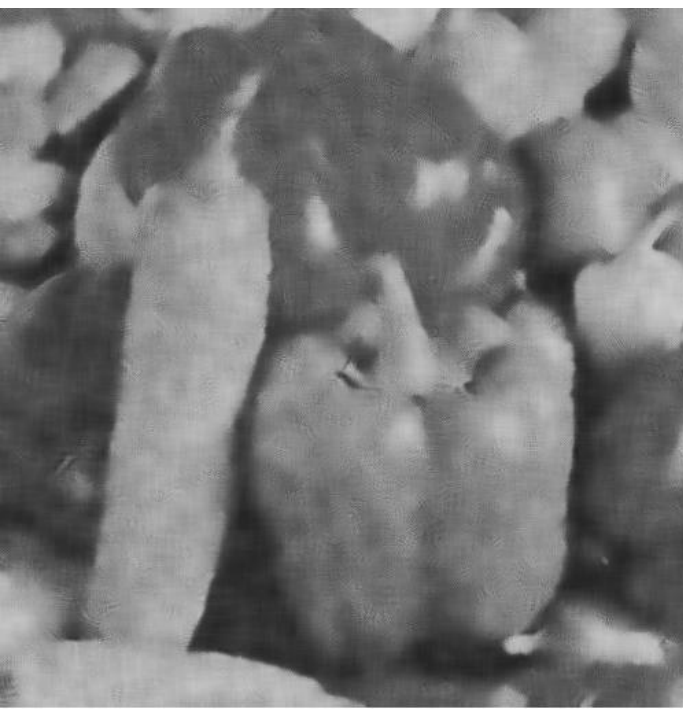

(d)

Figure A- 5: Subjective comparison for denoising performance for Non-Local Means at noise level $\sigma=100$. (a) noise free image Peppers. (b) noisy image with Additive white Gaussian noise. (c) denoised image using the NLM method, PSNR=21.4862, and (d) denoised image using the proposed method, PSNR $=21.7658$. 
Table A- 3: PSNR (dB) comparison for Boat image among the proposed method, the NLM method, variants of the NLM method and the BM3D method for different noise levels.

\begin{tabular}{|c|c|c|c|c|c|}
\hline Noise Level & NLM & PCA-NLM & NLM-Patch & $\begin{array}{c}\text { Proposed } \\
\text { Method } \\
\end{array}$ & BM3D \\
\hline \multicolumn{6}{|c|}{ Boat } \\
\hline 10 & 32.28 & 33.92 & 33.37 & 34.04 & 33.86 \\
\hline 20 & 29.62 & 29.90 & 30.11 & 30.72 & 30.71 \\
\hline 30 & 27.85 & 28.69 & 27.82 & 29.02 & 29.01 \\
\hline 40 & 26.37 & 26.07 & 25.74 & 27.43 & 27.60 \\
\hline 50 & 25.14 & 25.06 & 25.23 & 26.34 & 26.38 \\
\hline 60 & 23.88 & 23.88 & 23.93 & 24.78 & 26.02 \\
\hline 70 & 22.45 & 22.28 & 23.04 & 22.98 & 25.40 \\
\hline 80 & 22.07 & 22.12 & 22.69 & 22.19 & 24.86 \\
\hline 90 & 21.41 & 20.44 & 21.89 & 21.52 & 24.39 \\
\hline 100 & 20.91 & 20.16 & 21.04 & 20.95 & 23.97 \\
\hline Average & 25.20 & 25.25 & 25.48 & 25.99 & 27.22 \\
\hline
\end{tabular}


Table A- 4: SSIM comparison for Boat image among the proposed method, the NLM method, variants of the NLM method and the BM3D method for different noise levels.

\begin{tabular}{|c|c|c|c|c|c|}
\hline Noise Level & NLM & PCA-NLM & NLM-Patch & $\begin{array}{c}\text { Proposed } \\
\text { Method } \\
\end{array}$ & BM3D \\
\hline \multicolumn{6}{|c|}{ Boat } \\
\hline 10 & 0.9381 & 0.9315 & 0.9142 & 0.9452 & 0.9331 \\
\hline 20 & 0.8772 & 0.8669 & 0.8632 & 0.8843 & 0.8792 \\
\hline 30 & 0.8409 & 0.8396 & 0.8368 & 0.8471 & 0.8412 \\
\hline 40 & 0.7864 & 0.7813 & 0.7839 & 0.7924 & 0.8012 \\
\hline 50 & 0.7561 & 0.7527 & 0.7512 & 0.7669 & 0.7715 \\
\hline 60 & 0.7357 & 0.7361 & 0.7412 & 0.7469 & 0.7492 \\
\hline 70 & 0.7291 & 0.7245 & 0.7184 & 0.7311 & 0.7395 \\
\hline 80 & 0.7113 & 0.7059 & 0.7044 & 0.7195 & 0.7251 \\
\hline 90 & 0.7045 & 0.6998 & 0.7043 & 0.7096 & 0.7186 \\
\hline 100 & 0.6845 & 0.6673 & 0.6697 & 0.6982 & 0.7105 \\
\hline Average & 0.7970 & 0.7901 & 0.7874 & 0.8046 & 0.8065 \\
\hline
\end{tabular}




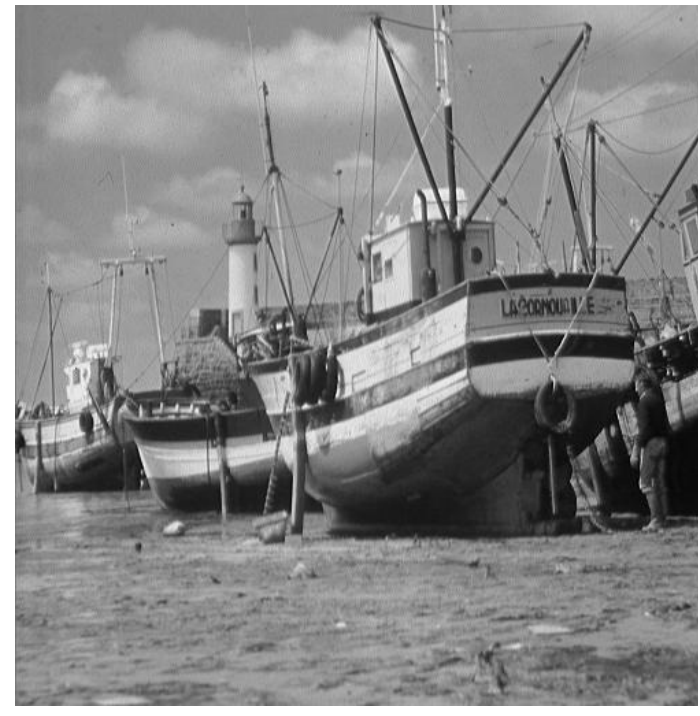

(a)

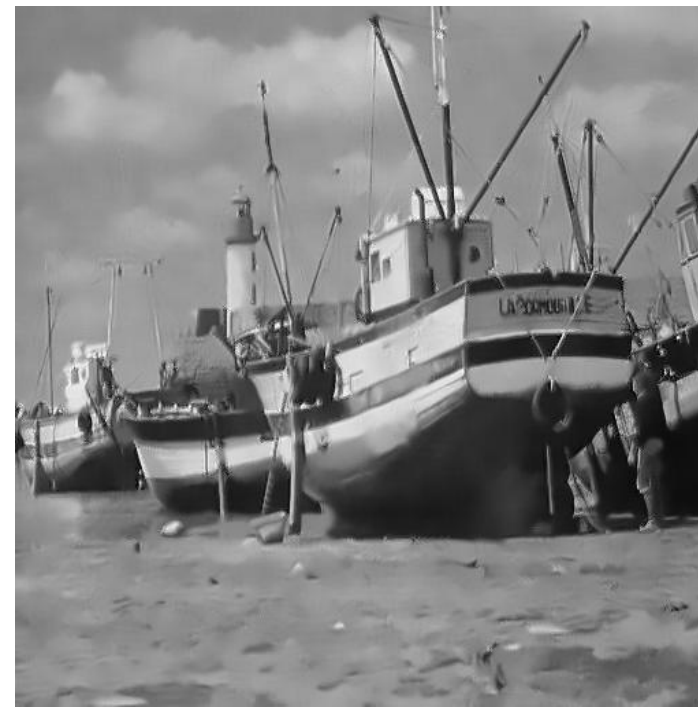

(c)

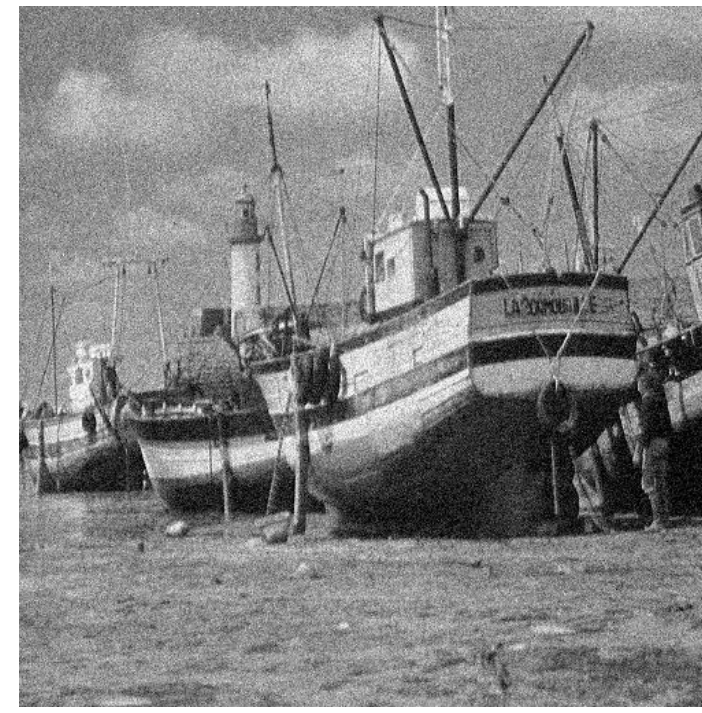

(b)

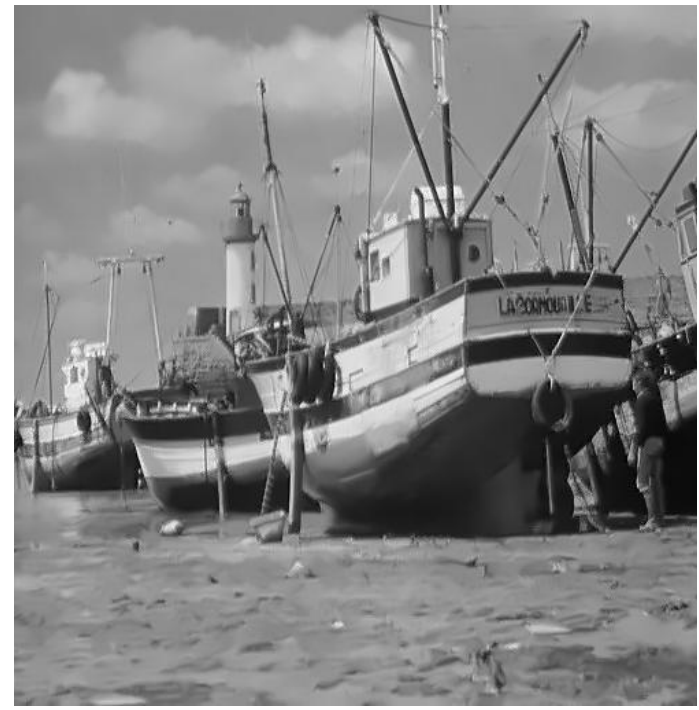

(d)

Figure A- 6: Subjective comparison for denoising performance for Non-Local Means at noise level $\sigma=20$. (a) noise free image Boat. (b) noisy image with Additive white Gaussian noise. (c) denoised image using the NLM method, PSNR=29.6207, and (d) denoised image using the proposed method, PSNR $=30.7237$. 


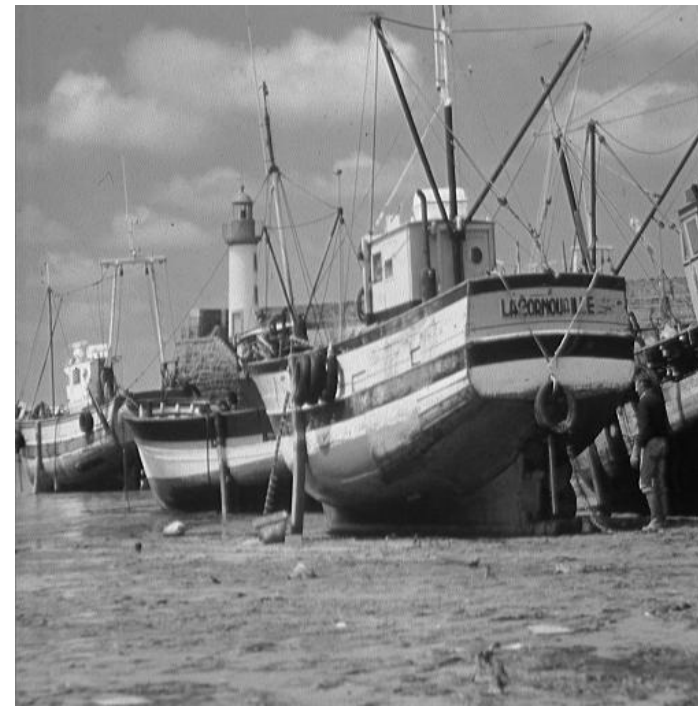

(a)

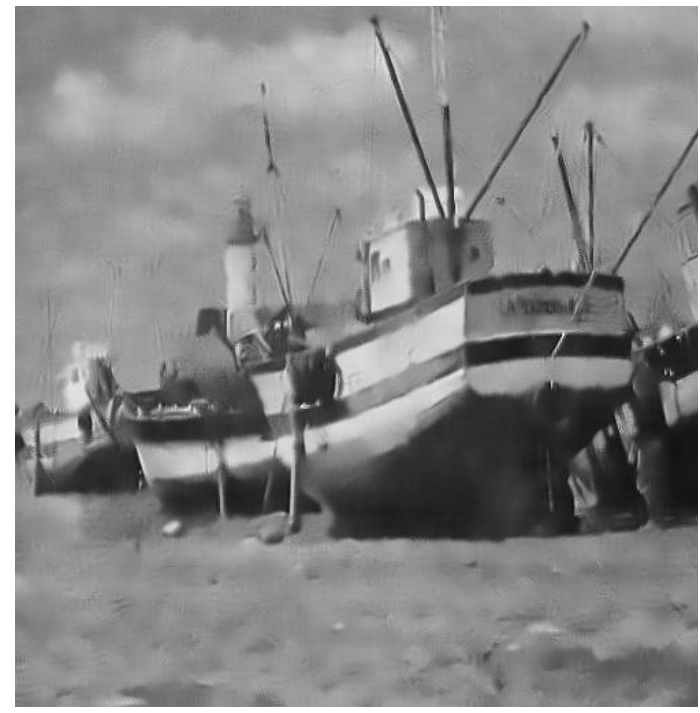

(c)

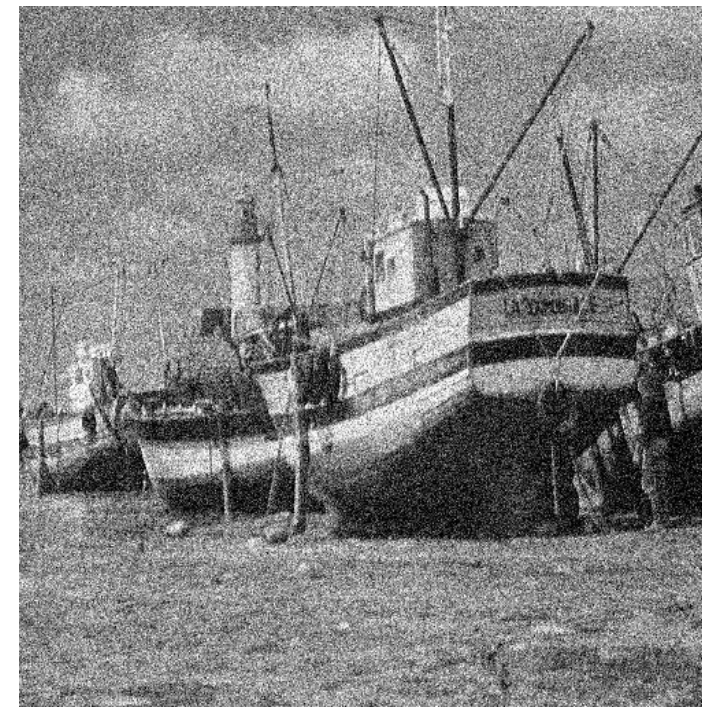

(b)

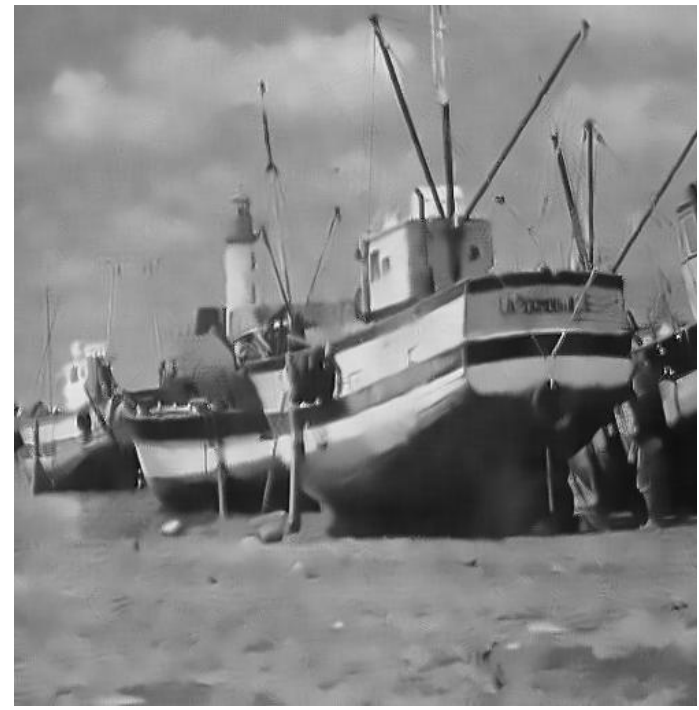

(d)

Figure A- 7: Subjective comparison for denoising performance for Non-Local Means at noise level $\sigma=40$. (a) noise free image Boat. (b) noisy image with Additive white Gaussian noise. (c) denoised image using the NLM method, PSNR= 26.3797, and (d) denoised image using the proposed method, PSNR $=27.4276$. 


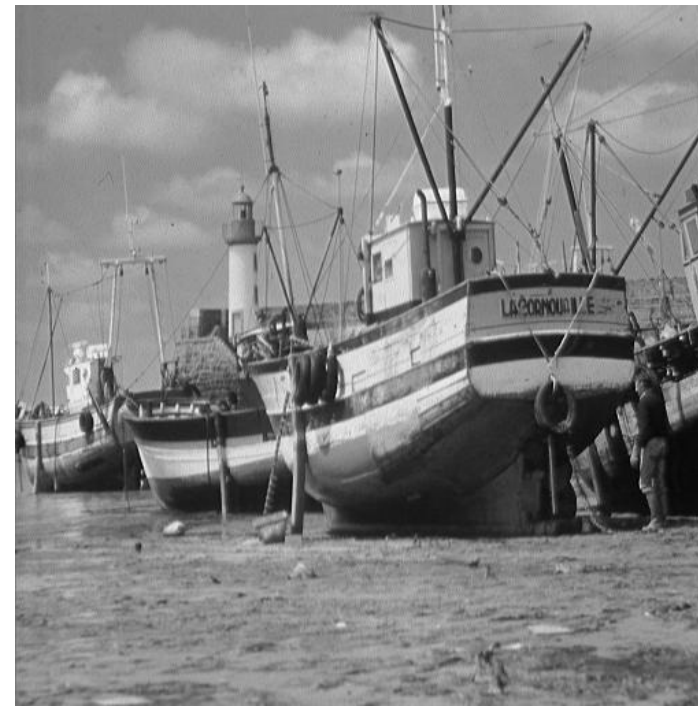

(a)

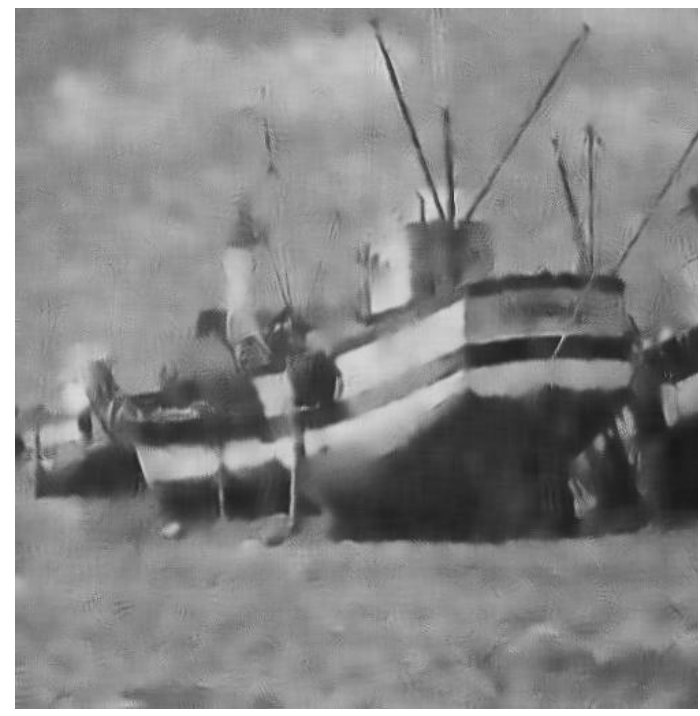

(c)

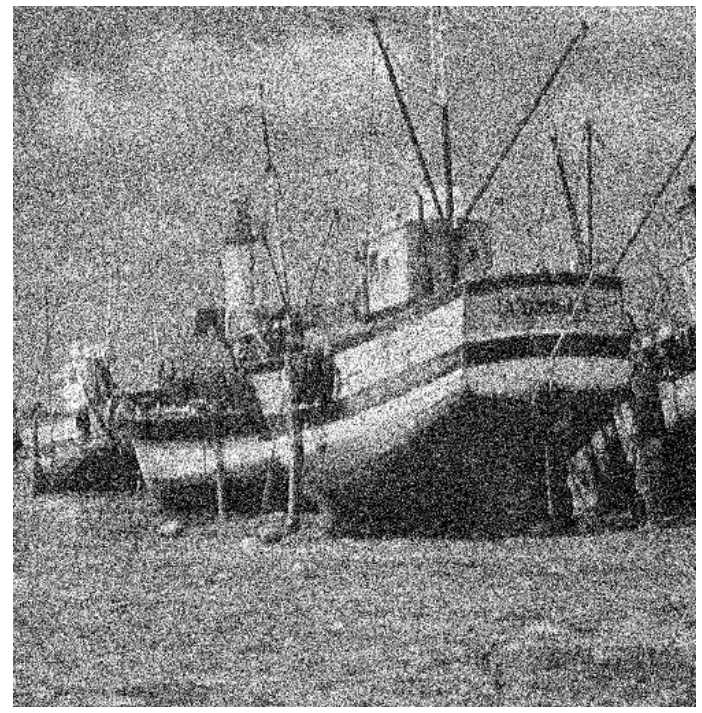

(b)

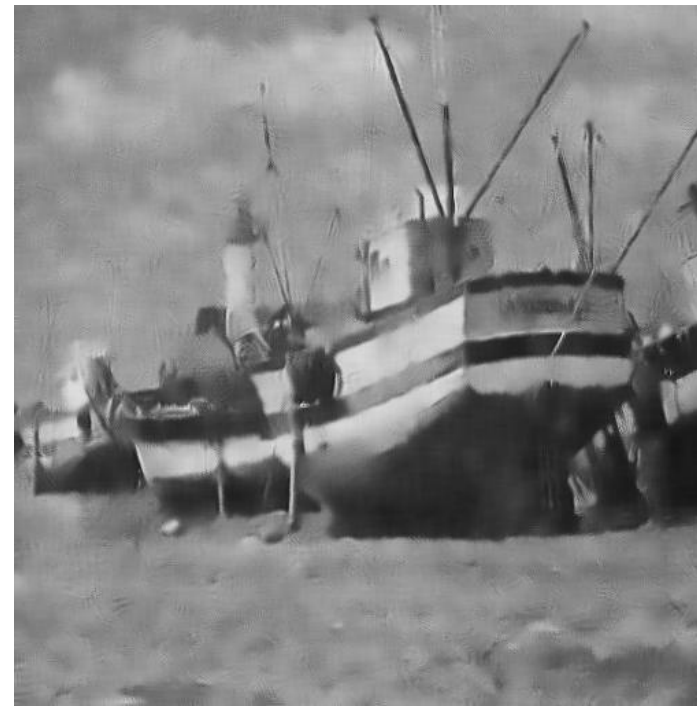

(d)

Figure A- 8: Subjective comparison for denoising performance for Non-Local Means at noise level $\sigma=60$. (a) noise free image Boat. (b) noisy image with Additive white Gaussian noise. (c) denoised image using the NLM method, PSNR= 23.8791, and (d) denoised image using the proposed method, PSNR $=24.7791$. 


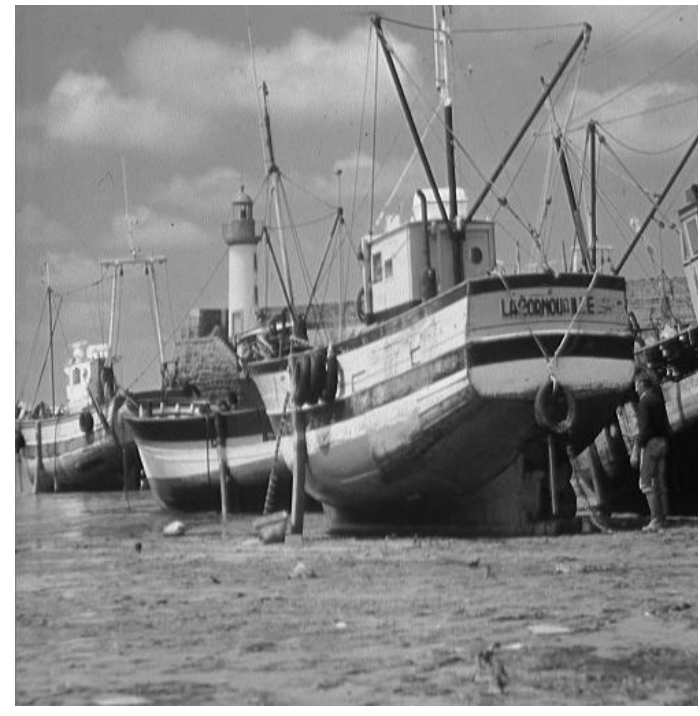

(a)

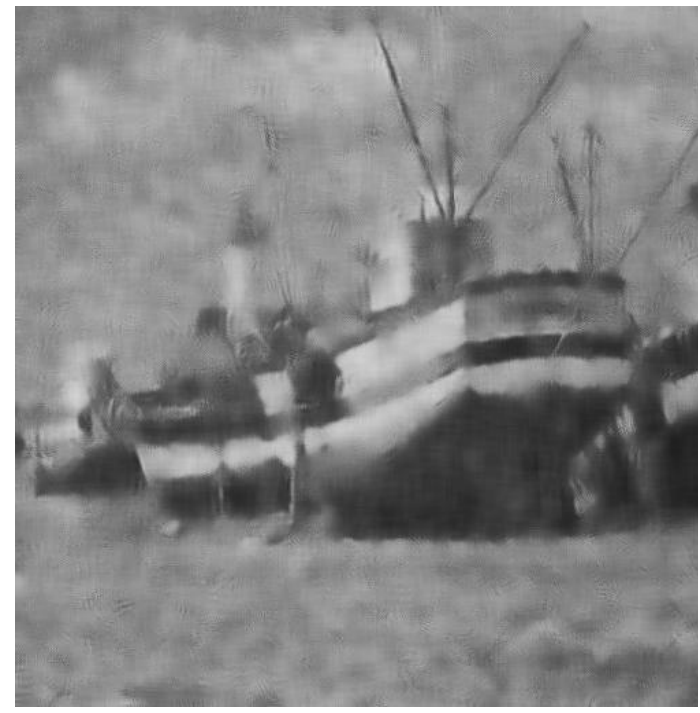

(c)

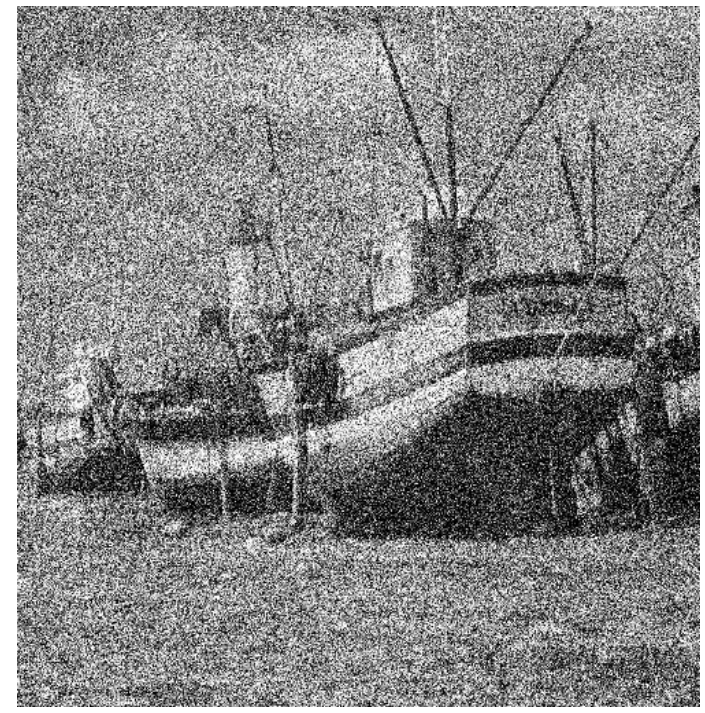

(b)

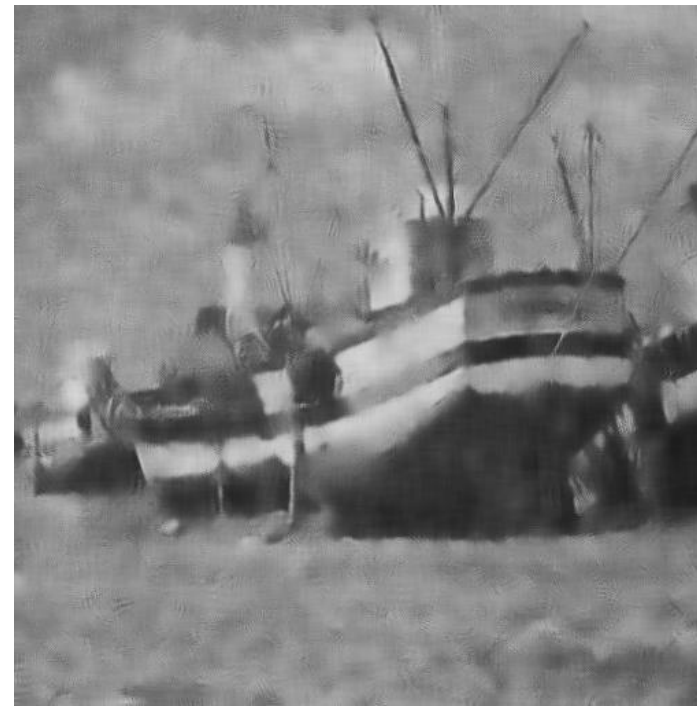

(d)

Figure A- 9: Subjective comparison for denoising performance for Non-Local Means at noise level $\sigma=80$. (a) noise free image Boat. (b) noisy image with Additive white Gaussian noise. (c) denoised image using the NLM method, PSNR= 22.0718, and (d) denoised image using the proposed method, PSNR $=22.1941$. 


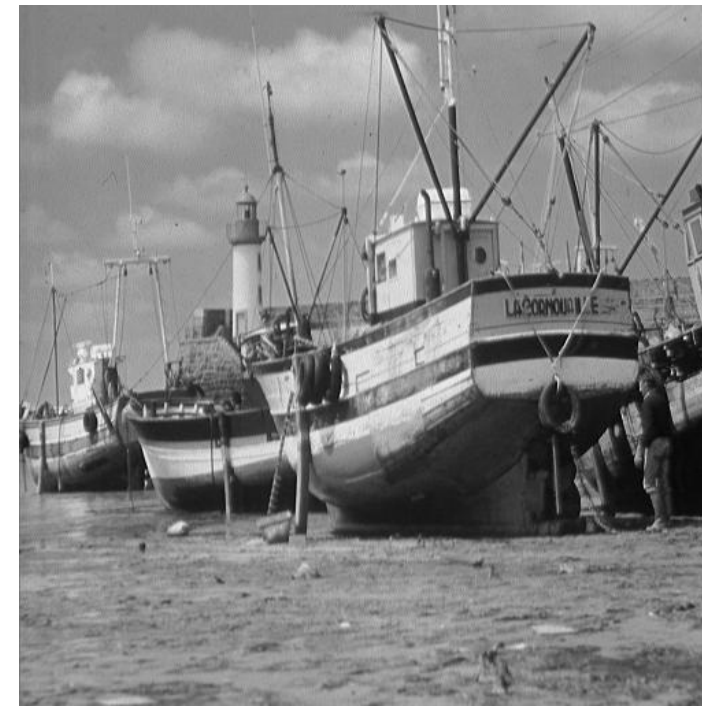

(a)

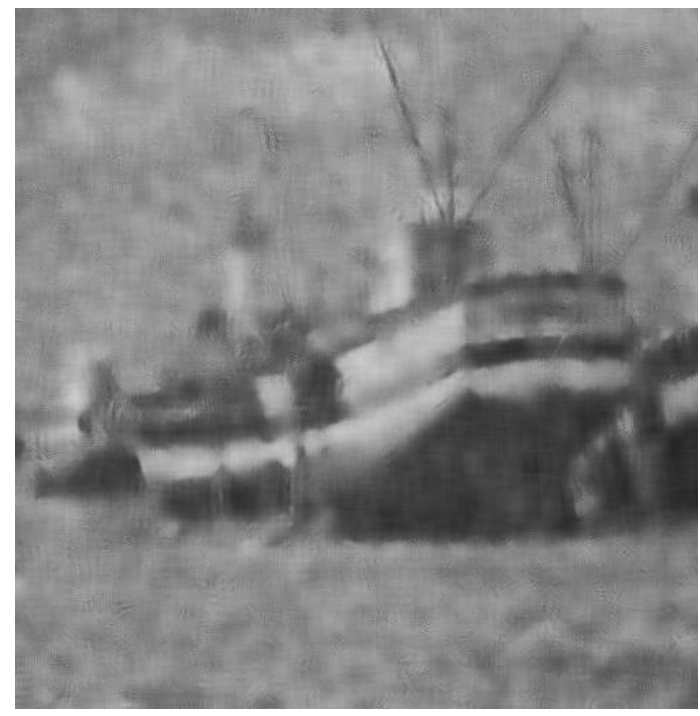

(c)

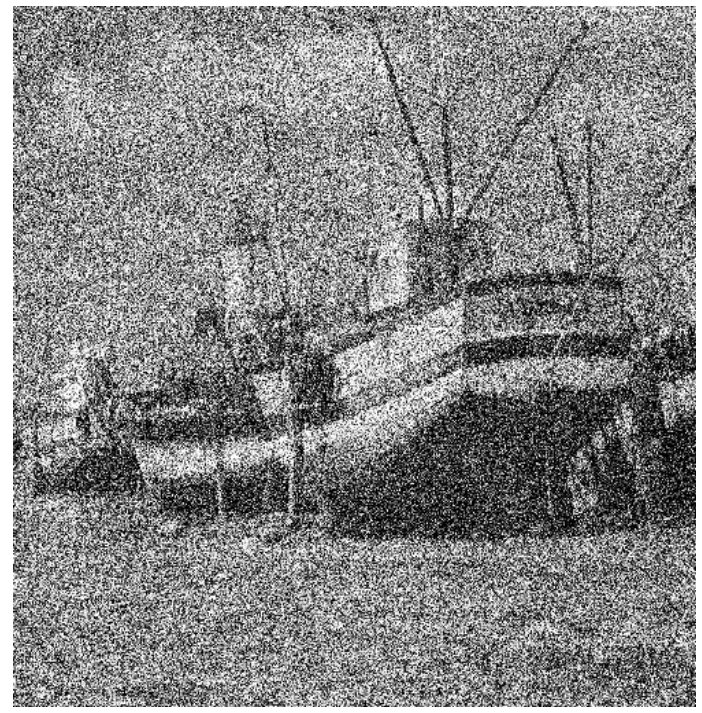

(b)

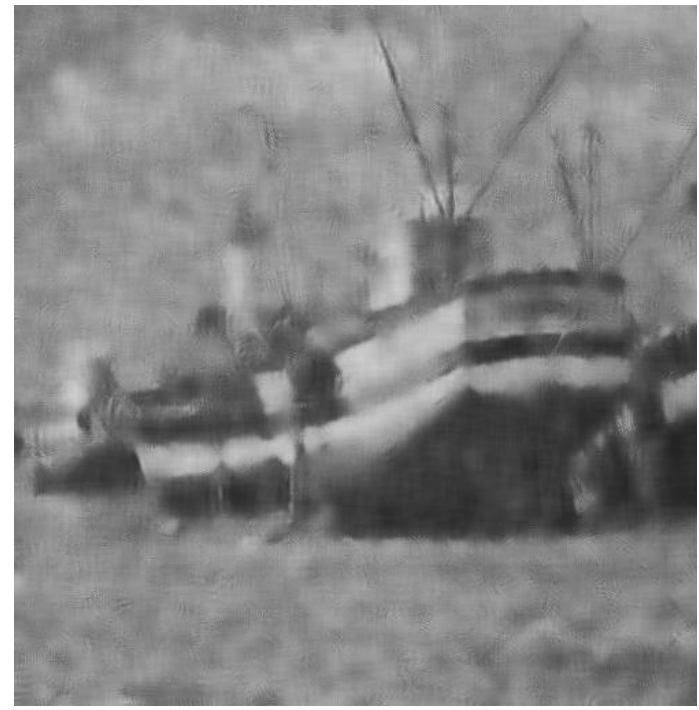

(d)

Figure A- 10: Subjective comparison for denoising performance for Non-Local Means at noise level $\sigma=100$. (a) noise free image Boat. (b) noisy image with Additive white Gaussian noise. (c) denoised image using the NLM method, PSNR= 20.9146, and (d) denoised image using the proposed method, PSNR $=20.9538$. 
Table A- 5: PSNR (dB) comparison for Couple image among the proposed method, the NLM method, variants of the NLM method and the BM3D method for different noise levels.

\begin{tabular}{|c|c|c|c|c|c|}
\hline Noise Level & NLM & PCA-NLM & NLM-Patch & $\begin{array}{c}\text { Proposed } \\
\text { Method } \\
\end{array}$ & BM3D \\
\hline \multicolumn{6}{|c|}{ Couple } \\
\hline 10 & 33.81 & 32.91 & 33.43 & 33.83 & 33.73 \\
\hline 20 & 30.14 & 29.87 & 30.23 & 30.81 & 30.78 \\
\hline 30 & 27.98 & 27.05 & 27.83 & 28.35 & 28.87 \\
\hline 40 & 26.18 & 25.00 & 25.59 & 26.79 & 27.48 \\
\hline 50 & 25.54 & 24.71 & 25.14 & 25.62 & 26.46 \\
\hline 60 & 23.18 & 23.01 & 23.78 & 23.31 & 26.02 \\
\hline 70 & 22.45 & 22.17 & 23.07 & 22.55 & 25.40 \\
\hline 80 & 21.83 & 21.14 & 22.91 & 21.92 & 24.86 \\
\hline 90 & 21.12 & 21.08 & 21.84 & 21.38 & 24.39 \\
\hline 100 & 21.00 & 21.01 & 21.27 & 21.00 & 23.97 \\
\hline Average & 25.34 & 24.79 & 25.50 & 25.53 & 27.22 \\
\hline
\end{tabular}


Table A- 6: SSIM comparison for Couple image among the proposed method, the NLM method, variants of the NLM method and the BM3D method for different noise levels.

\begin{tabular}{|c|c|c|c|c|c|}
\hline Noise Level & NLM & PCA-NLM & NLM-Patch & $\begin{array}{c}\text { Proposed } \\
\text { Method } \\
\end{array}$ & BM3D \\
\hline \multicolumn{6}{|c|}{ Couple } \\
\hline 10 & 0.9132 & 0.9124 & 0.9073 & 0.9352 & 0.9278 \\
\hline 20 & 0.8817 & 0.8698 & 0.8685 & 0.8917 & 0.8892 \\
\hline 30 & 0.8492 & 0.8397 & 0.8388 & 0.8501 & 0.8463 \\
\hline 40 & 0.8077 & 0.8036 & 0.7982 & 0.8138 & 0.8194 \\
\hline 50 & 0.7414 & 0.7431 & 0.7412 & 0.7589 & 0.7714 \\
\hline 60 & 0.7387 & 0.7328 & 0.7391 & 0.7412 & 0.7467 \\
\hline 70 & 0.7257 & 0.7209 & 0.7187 & 0.7327 & 0.7389 \\
\hline 80 & 0.7084 & 0.7025 & 0.7071 & 0.7124 & 0.7334 \\
\hline 90 & 0.6912 & 0.6897 & 0.7039 & 0.6845 & 0.7148 \\
\hline 100 & 0.6781 & 0.6686 & 0.6654 & 0.6823 & 0.6987 \\
\hline Average & 0.79392 & 0.78877 & 0.78774 & 0.80103 & 0.80871 \\
\hline
\end{tabular}




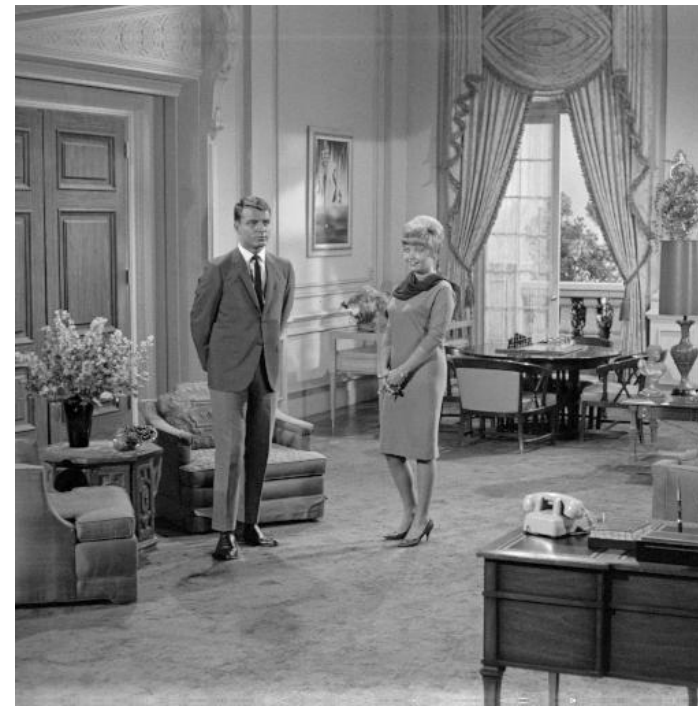

(a)

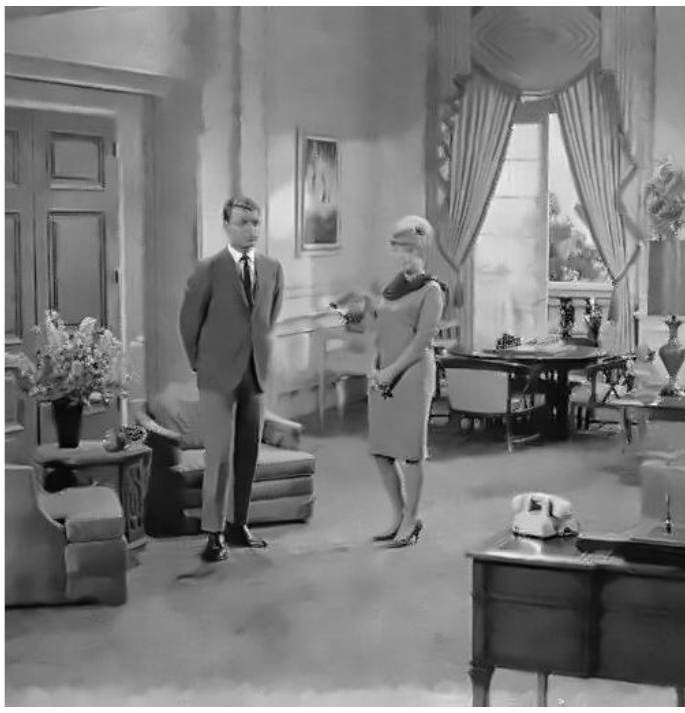

(c)

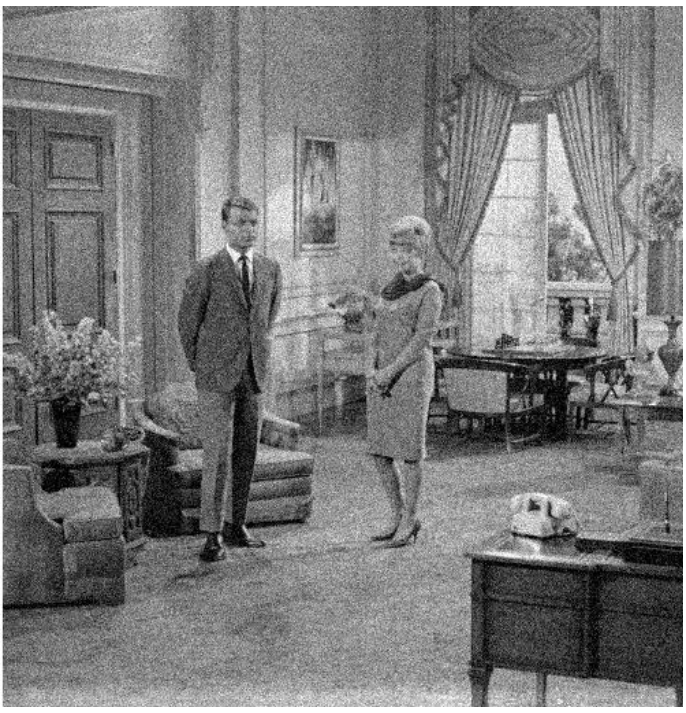

(b)

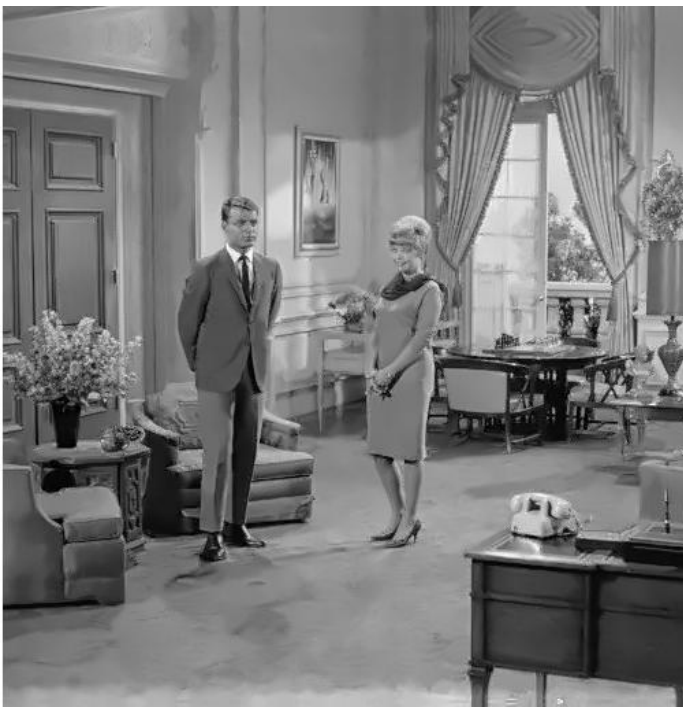

(d)

Figure A- 11: Subjective comparison for denoising performance for Non-Local Means at noise level $\sigma=20$. (a) noise free image Couple.(b) noisy image with Additive white Gaussian noise, (c) denoised image using the NLM method, PSNR= 30.1471, and (d) denoised image using the proposed method, PSNR $=30.8116$. 


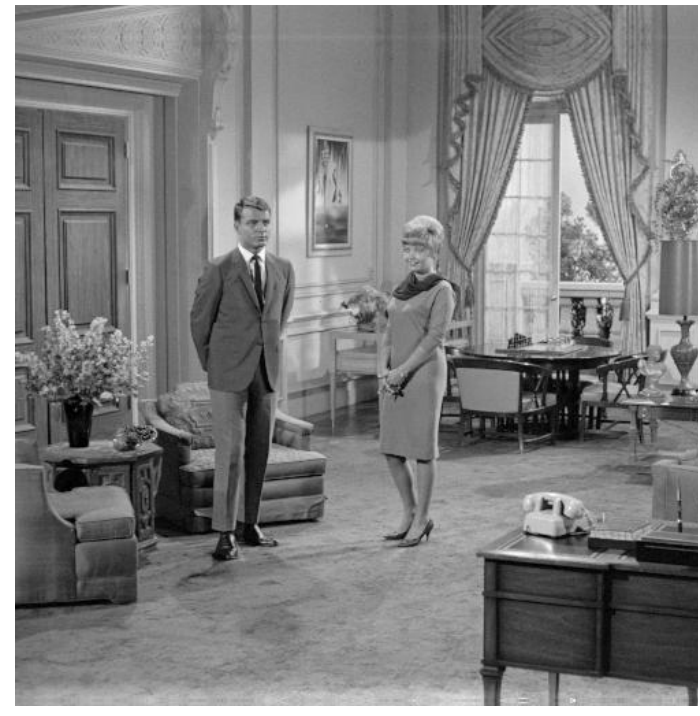

(a)

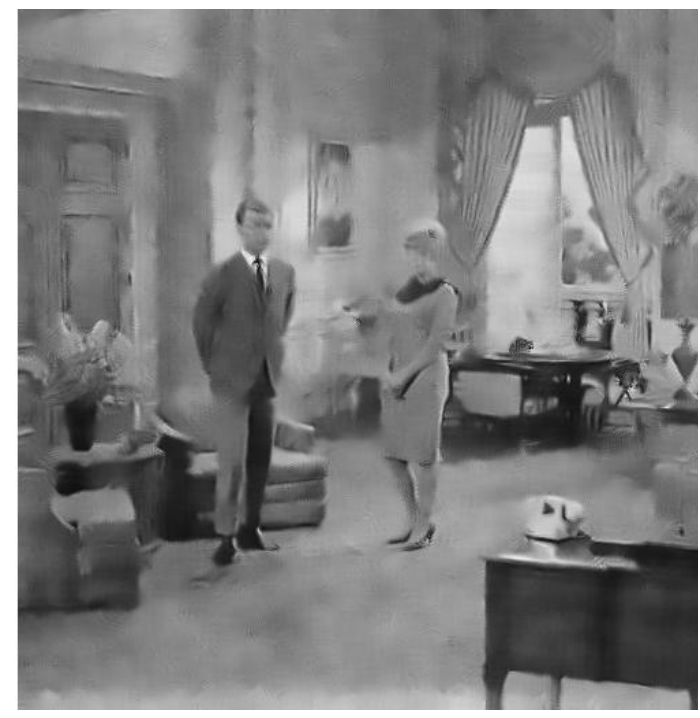

(c)

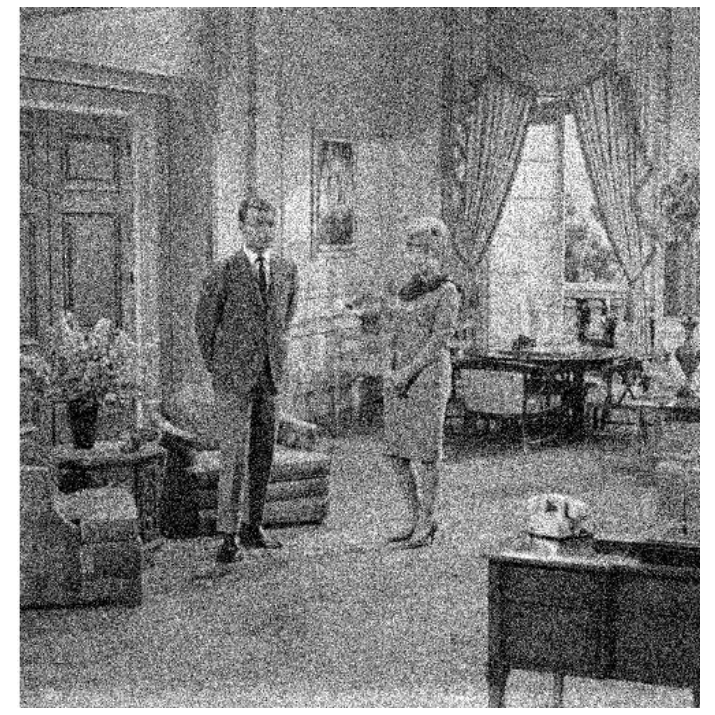

(b)

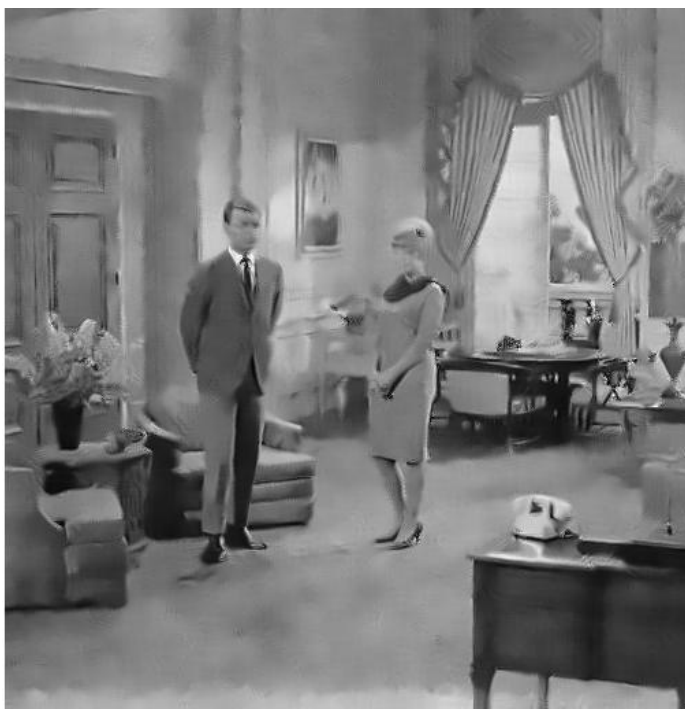

(d)

Figure A- 12: Subjective comparison for denoising performance for Non-Local Means at noise level $\sigma=40$. (a) noise free image Couple. (b) noisy image with Additive white Gaussian noise. (c) denoised image using the NLM method, PSNR= 26.1758, , and (d) denoised image using the proposed method, PSNR $=26.7854$. 


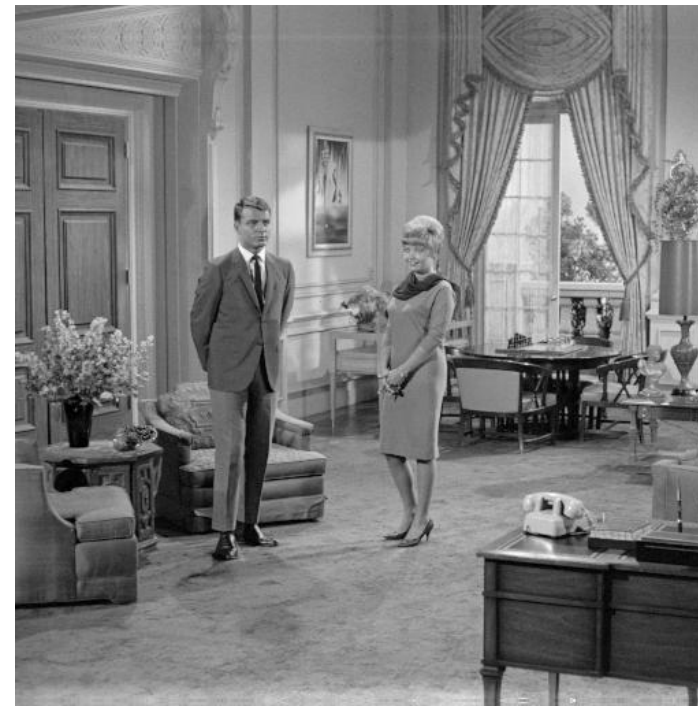

(a)

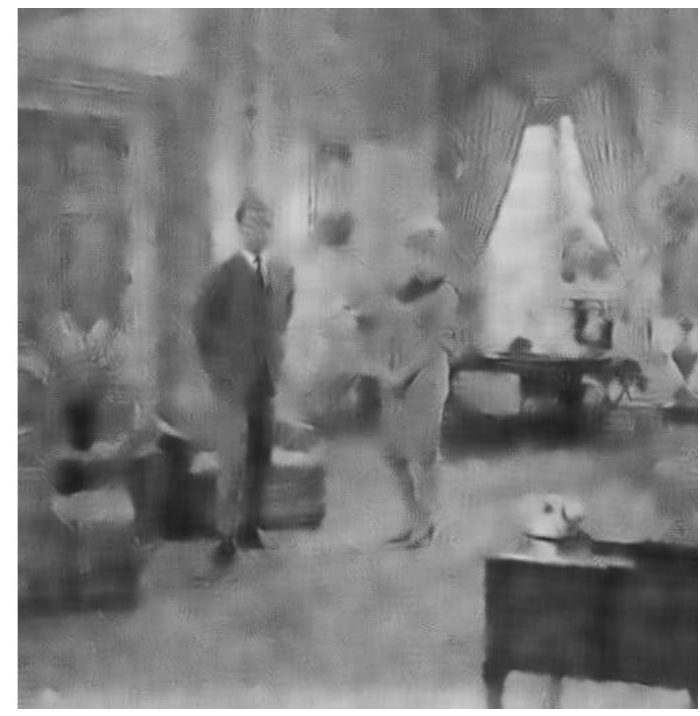

(c)

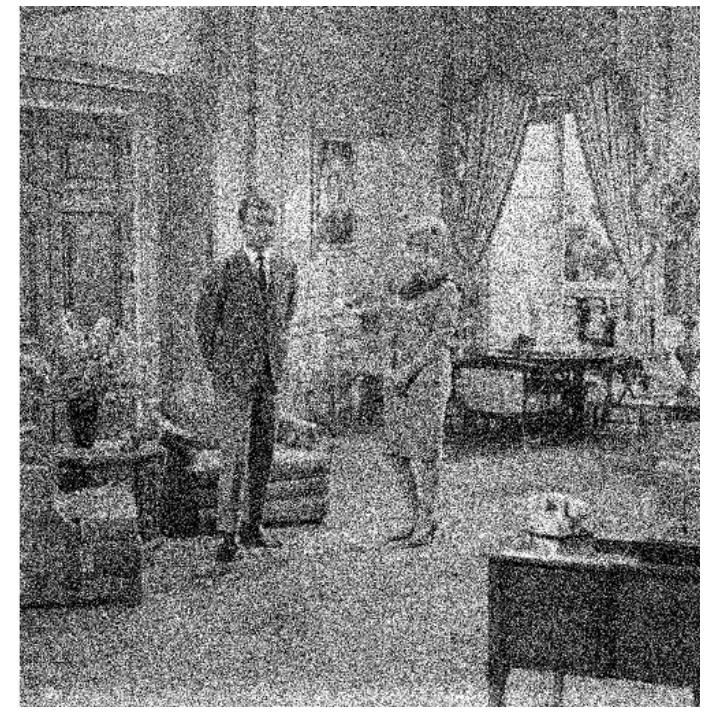

(b)

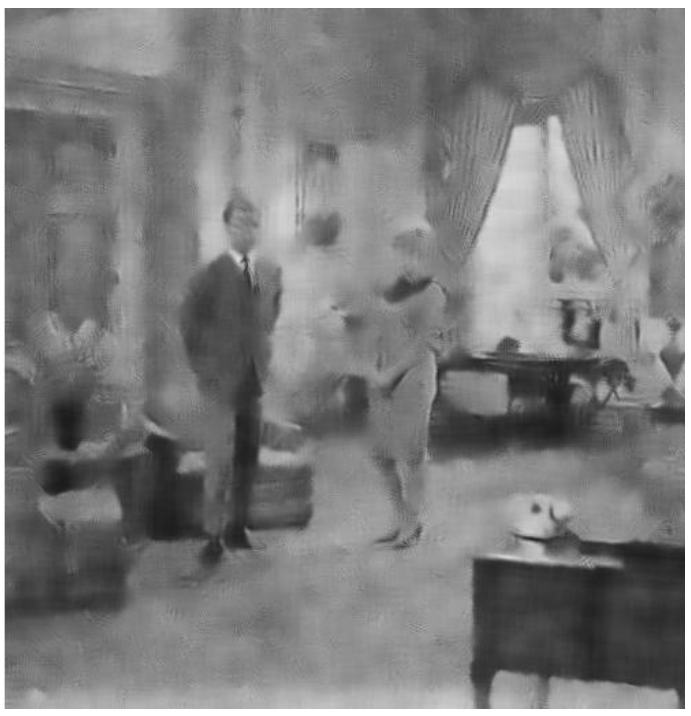

(d)

Figure A- 13: Subjective comparison for denoising performance for Non-Local Means at noise level $\sigma=60$. (a) noise free image Couple. (b) noisy image with Additive white Gaussian noise. (c) denoised image using the NLM method, PSNR= 23.1847, and (d) denoised image using the proposed method, PSNR $=23.3052$. 


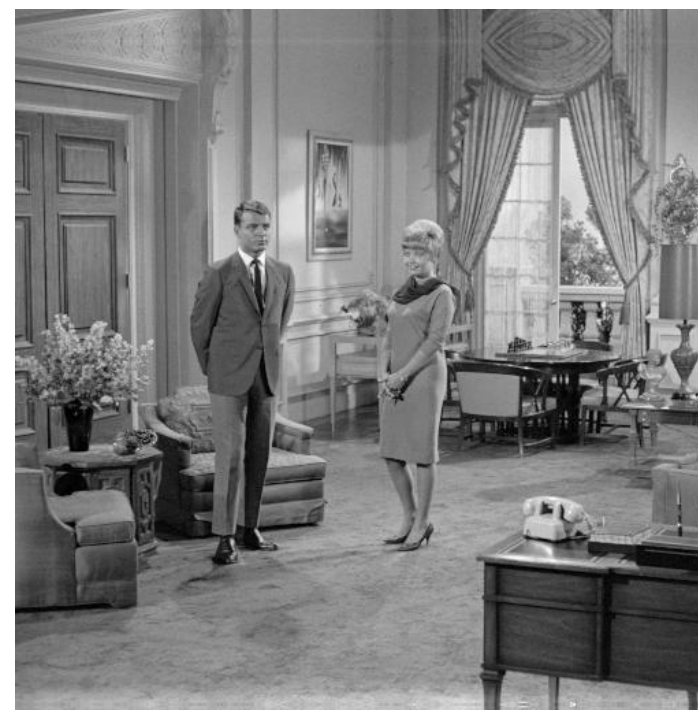

(a)

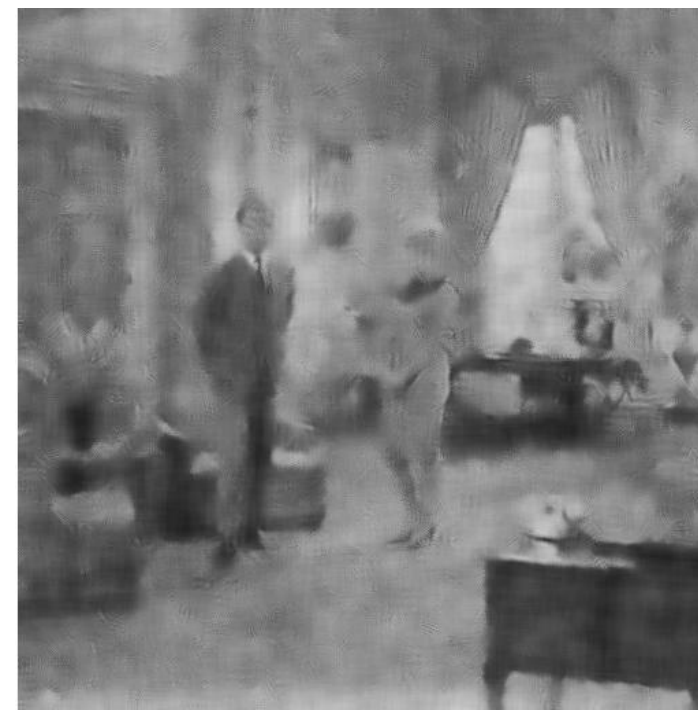

(c)

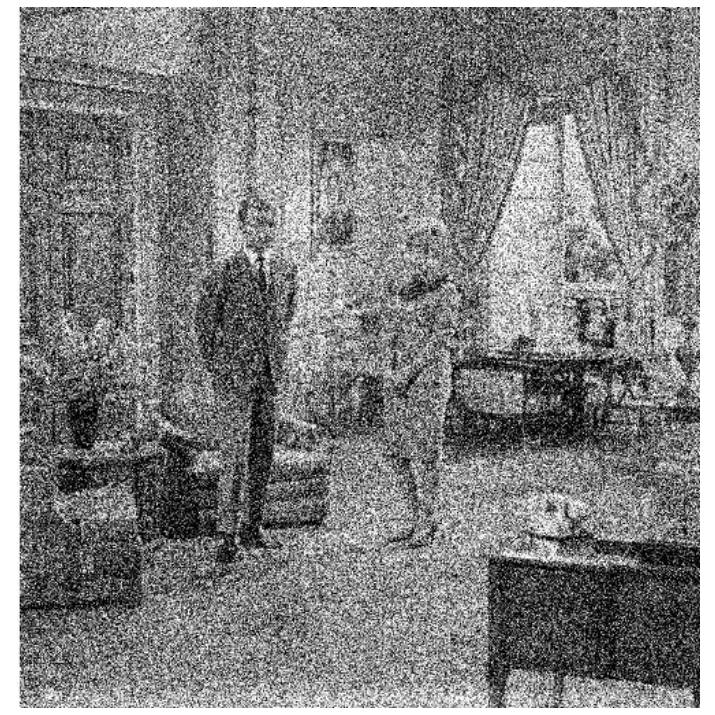

(b)

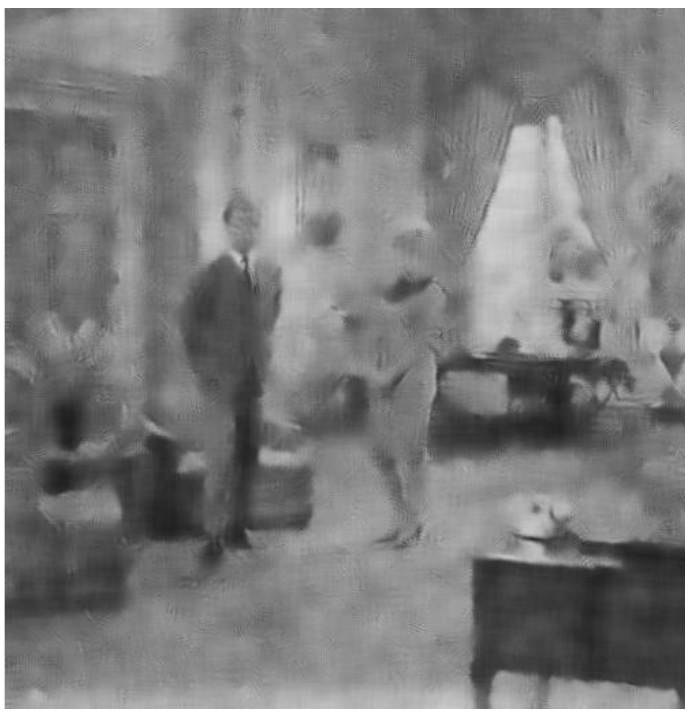

(d)

Figure A- 14: Subjective comparison for denoising performance for Non-Local Means at noise level $\sigma=80$. (a) noise free image Couple. (b) noisy image with Additive white Gaussian noise. (c) denoised image using the NLM method, PSNR= 21.8258, and (d) denoised image using the proposed method, PSNR $=21.9186$. 


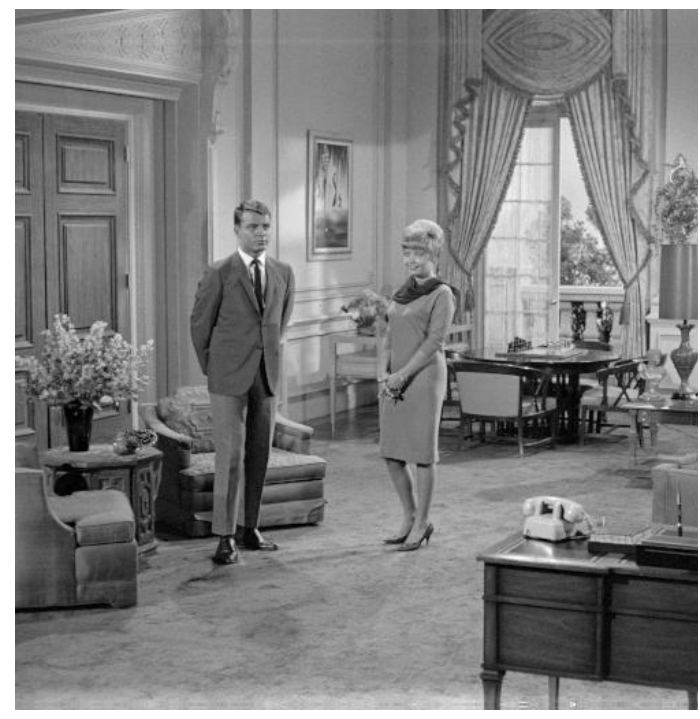

(a)

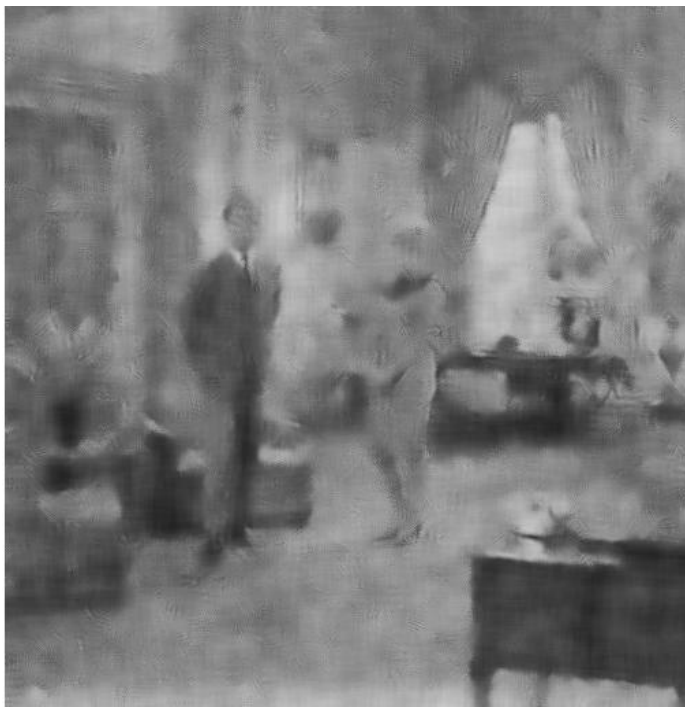

(c)

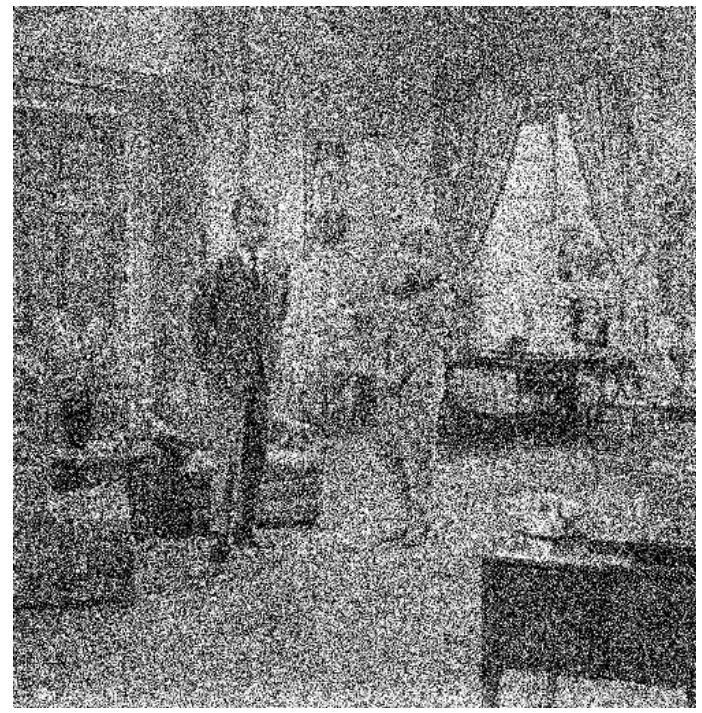

(b)

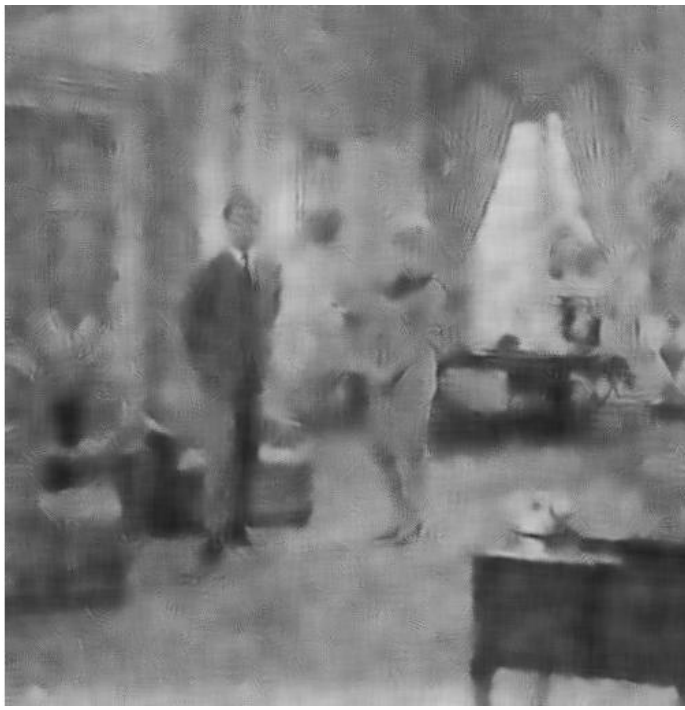

(d)

Figure A- 15: Subjective comparison for denoising performance for Non-Local Means at noise level $\sigma=100$. (a) noise free image Couple. (b) noisy image with Additive white Gaussian noise. (c) denoised image using the NLM method, PSNR= 21.0014, and (d) denoised image using the proposed method, PSNR $=21.0033$. 


\section{Curriculum Vitae}

Name:

Post-secondary

Education and

Degrees:

Honours and

Awards:

Related Work

Experience
Golam Morshed Maruf

Islamic University of Technology

Dhaka, Bangladesh

2007-2010

B.Sc.

The University of Western Ontario

London, Ontario, Canada

2013-2014

M.Sc.
Western University Graduate Research Scholarship 2013-2014,

Organization of Islamic Conference (OIC) Scholarship 2007-2010

Teaching Assistant

The University of Western Ontario

2013-2014

Research Assistant

The University of Western Ontario

2013-2014 